\title{
TIME-FREQUENCY SIGNAL SYNTHESIS AND ITS \\ APPLICATION IN MULTIMEDIA WATERMARK DETECTION
}

by

\section{LAM LE}

B.Sc., University of Saigon, Saigon, Vietnam, 1991

B.Eng., Ryerson University, Toronto, Canada, 2003

\author{
A thesis \\ presented to Ryerson University \\ in partial fulfillment of the \\ requirement for the degree of \\ Master of Applied Science \\ in the Program of
}

Electrical and Computer Engineering

Toronto, Ontario, Canada, 2005

(C) LAM LE, 2005 
UMI Number: EC53435

\section{INFORMATION TO USERS}

The quality of this reproduction is dependent upon the quality of the copy submitted. Broken or indistinct print, colored or poor quality illustrations and photographs, print bleed-through, substandard margins, and improper alignment can adversely affect reproduction.

In the unlikely event that the author did not send a complete manuscript and there are missing pages, these will be noted. Also, if unauthorized copyright material had to be removed, a note will indicate the deletion.

\section{UMI}

UMI Microform EC53435

Copyright 2009 by ProQuest LLC

All rights reserved. This microform edition is protected against unauthorized copying under Title 17, United States Code.

ProQuest LLC

789 East Eisenhower Parkway

P.O. Box 1346

Ann Arbor, MI 48106-1346 


\section{Author's Declaration}

I hereby declare that I am the sole author of this thesis.

I authorize Ryerson University to lend this thesis to other institutions or individuals for the purpose of scholarly research.

Signature

I further authorize Ryerson University to reproduce this thesis by photocopying or by other means, in total or in part, at the request of other institutions or individuals for the purpose of scholarly research.

Signature 


\section{Instructions on Borrowers}

Ryerson University requires the signatures of all persons using or photocopying this thesis. Please sign below, and give address and date. 


\title{
Abstract \\ Time-Frequency Signal Synthesis and Its Application In Multimedia Watermark Detection \\ (C)Lam Le, 2005 \\ Master of Applied Science \\ Department of Electrical and Computer Engineering \\ Ryerson University
}

\begin{abstract}
A novel approach is proposed in this thesis to synthesize the time domain chirp signal from the joint time-frequency distribution (TFD). The objective is to reconstruct the original signal from its corrupted version. The new signal synthesis technique is based on the Discrete Polynomial Phase Transform (DPPT) and the TFD of the signal to be synthesized. The TFD is used to separate the mono-component signals from a multi-component signal. The DPPT is then applied on the estimated mono-components to have a final synthesized version of the individual time domain signals.

The candidate TFD to be used in the synthesis technique is chosen from a group of common TFDs based on their performance with different types of signals. The criteria for the comparison are joint time-frequency localization, low susceptibility to noise, crossterm suppression and the precision of the instantaneous frequency estimated from these distributions. Smoothed Pseudo Wigner-Ville Distribution is chosen as the processing TFD in the proposed signal synthesis technique.

The proposed chirp synthesis technique is applied to detect the presence of the chirp signal embedded as a watermark message in multimedia security applications. The technique can detect the presence of chirp signals from a corrupted chirp with a bit error rate up to $15 \%$. Computational complexity of the proposed chirp detection based on time-frequency signal synthesis is proved to be less than that of the detection method based on the Hough Radon Transform and the proposed signal synthesis technique may also be used as an error correction tool in other applications.
\end{abstract}




\section{Acknowledgments}

I would like to express my sincere appreciation to those who have assisted and supported me during my study period. Particularly, I would like to thank my supervisor Dr. Krishnan for his enormous help, constant support and exceptional guidance over the years throughout my undergraduate and graduate studies. I acknowledge with gratitude the invaluable encouragement, feedback and time that he has given me especially at the final stages of the thesis.

I would also like to thank the Department of Electrical and Computer Engineering of Ryerson University and Communications and Information Technology Ontario (CITO) for providing me great support over the years.

I thank my thesis committee members for their time, patience and valuable feedback.

I would like to especially thank my family for their nonstop and wonderful support over the years. Many thanks go to Karthi Umapathy, Jiming Yang, Arun Ramalingam, all other members of the SAR group and friends for their kind assistance and inspiration.

I would like to acknowledge Francois Auger, Patrick Flandrin, Olivier Lemoine, and Paulo Goncalves from CNRS France and Rice University for developing the Time-Frequency Toolbox. 


\section{Contents}

1 Introduction $\quad 1$

2 Time-Frequency Localization Analysis 9

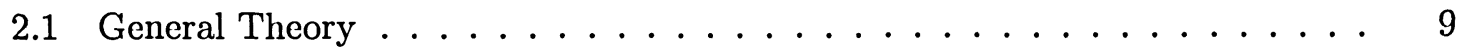

2.2 Cohen's Class of TFD . . . . . . . . . . . . . . . . . . . . . 11

2.2.1 Desired Properties of the TFD . . . . . . . . . . . . . . 11

2.2.2 Bi-linearity and Interference Term . . . . . . . . . . . . 13

2.2.3 The Analytic Signal and Spectral Aliasing . . . . . . . . . . . . 14

2.3 The Ambiguity Domain and the Trade-off Between the Joint Time-Frequency

Resolution and the Ability To Remove Interference Terms . . . . . . . . 15

2.4 Smoothing Kernels of the Popular TFDs . . . . . . . . . . . . . . 18

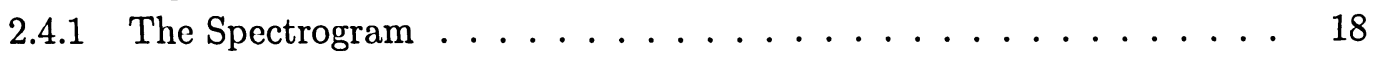

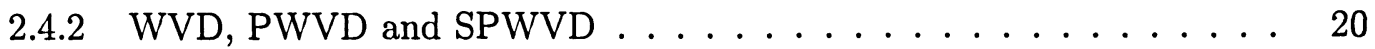

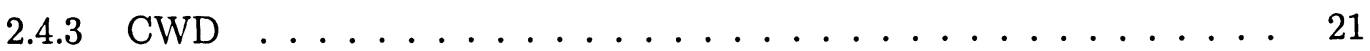

2.5 Performance Comparison Among The TFD's . . . . . . . . . . . . . . 22

2.5.1 Mono-component versus Multi-component Signal Simulation . . . . . 24

2.5.2 Simulation in White Noise . . . . . . . . . . . . . . 25

2.5.3 Discussion on Simulation Result . . . . . . . . . . . . 25

3 Signal Synthesis $\quad 37$

3.1 An Overview of Signal Synthesis . . . . . . . . . . . . . . . 37

3.2 The Time-Frequency Synthesis Method Based on Least Squares Approximation 38

3.3 The Problem of Phase Reconstruction and The Need For Initial Information From The Signals . . . . . . . . . . . . . . . . . . 39

3.4 The Synthesis Method Based on The DPPT _ . . . . . . . . . . . 40

3.5 Synthesis of Chirp Signals . . . . . . . . . . . . . . . 43

4 Chirp Synthesis and Detectors $\quad 49$

4.1 The Proposed Signal Synthesis Technique . . . . . . . . . . . . . . . 49

4.2 The Available Chirp Detection Methods in Time-Frequency Domain . . . . 56 
5 Applications in Multimedia Security

5.1 Linear Frequency Modulated Chirp as a Watermark Message and Detection Methods . . . . . . . . . . . . . . . . . . . 59

$5.2 \quad$ HRT-Based Detection . . . . . . . . . . . . . . . . . . . . 62

5.3 Detection of The Watermark Message Using The Proposed Signal Synthesis Approach . . . . . . . . . . . . . . . . 68

5.4 Computational Complexity of the Chirp Detection Methods . . . . . . . 74

6 Conclusions

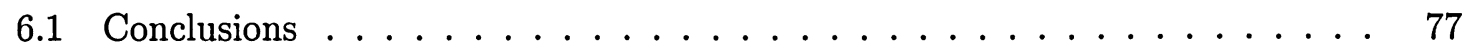

6.2 Related Future Work . . . . . . . . . . . . . . . . . . . 81

$\begin{array}{lc}\text { A List of Publications } & 83\end{array}$

B List of Acronyms $\quad 84$ 


\section{List of Figures}

1.1 The block diagram of the proposed system. $\ldots \ldots \ldots \ldots \ldots$

2.1 Location of interference terms and auto terms . . . . . . . . . 17

2.2 The shape of different TFDs kernel: a)WVD, (b)PWVD, (c) SPWVD, (d) Spectrogram (long window), (e) Spectrogram(short window), and (f) CWD . 19

2.3 CWD kernel . . . . . . . . . . . . . . . . . . . . 21

2.4 TFD of several popular estimators . . . . . . . . . . . . . . 23

2.5 Correlation coefficients between the estimated and referenced instantaneous frequency using the common TFDs . . . . . . . . . . . . . 30

2.6 Correlation coefficients between the estimated and referenced instantaneous frequency of different mono-component signals using the common TFDs under the effect of white uniform and white Gaussain noise . . . . . . . . . . .

2.7 Correlation coefficients between the estimated and referenced instantaneous frequency of different multi-component signals using the common TFDs under the effect of white uniform and white Gaussain noise . . . . . . . . . .

2.8 Correlation coefficients between the estimated and referenced instantaneous frequency of different multi-component signals using the common TFDs under the effect of white uniform and white Gaussain noise (Doppler signal) . . . .

3.1 DPPT of linear chirp signal with BER of $5 \% \ldots \ldots \ldots \ldots$. . . . . . . 42

3.2 Referenced (solid) and synthesized (dashed) versions of the linear chirp . . . 44

3.3 Referenced (solid) and synthesized (dashed) versions of the parabolic chirp . 45

3.4 Correlation coefficients between the synthesized and referenced chirps under the effect of white uniform and white Gaussian noise at various SNRs . . . 47

4.1 The MCE-TFD-based chirp detection method . . . . . . . . . . . 51

4.2 Effect of time-frequency filtering on instantaneous frequency estimation. 4.2(a): no time-frequency filtering: lower correlation (0.9861) between estimated (dashed) and referenced (solid) instantaneous frequency, and 4.2(b): with time-frequency filtering: high correlation (0.9993) between estimated (dashed) and referenced (solid) instantaneous frequency. The SNR of the original signal is $5 \mathrm{~dB} \quad \ldots \quad 52$

4.3 The proposed chirp detection method bases on the DPPT and SPWVD . . . 53

4.4 Synthesized (dashed) and referenced (solid) version of the individual component chirps of the multi-component signal $\ldots \ldots \ldots \ldots \ldots \ldots$ 
5.1 The process of embedding chirp message in audio/image file . . . . . . .

5.2 (a),(b): Image with no watermark embedded and its histogram, (c): time domain representation of the linear chirp (watermark), (d): TFD of the linear chirp, (e): the image in (a) with watermark embedded, and (f): its histogram. 64

5.3 The image space . . . . . . . . . . . . . . . . . 66

5.4 SPWVD and the corresponding HRT of mono-component and multi-component linear chirp signals . . . . . . . . . . . . . . . . 67

5.5 The watermark detection scheme . . . . . . . . . . . 68

5.6 (a): TFD of the chirp with $20 \%$ BER, and (b): time domain plot of the original chirp (solid) and synthesized chirp (dashed) corresponding to a correlation

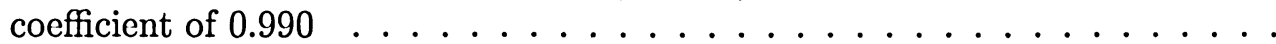

5.7 Correlation coefficients between the synthesized and referenced chirps in continuous and quantized forms for various length of the signals . . . . . . . .

5.8 (a): TFD of the chirp, (b): time domain plot of the original chirp (solid) and synthesized chirp (dashed) corresponding to a correlation coefficient of 0.94 at $5 \%$ BER, and (c): correlation coefficients at different BER between the original and synthesized chirp (solid), between their quantized versions (dashed), and between the quantized original chirp and quantized chirp at the receiver (dash-star) . . . . . . . . . . . . . . . .

5.9 Correlation coefficients between the synthesized and referenced chirps in continuous and quantized forms with and without the instantaneous frequency estimation process at different BERs $\ldots \ldots \ldots \ldots \ldots \ldots$

6.1 (a): TFD of the chirp with discontinuity in its instantaneous frequency law, and (b): time domain plot of the original chirp (solid) and synthesized chirp (dashed) corresponding to a correlation coefficient of $0.9912 \ldots \ldots$. . . 


\section{List of Tables}

2.1 Smoothing kernels of the common TFDs . . . . . . . . . . . .

2.2 Correlation coefficients between the estimated and referenced instantaneous frequency resulted from various time-frequency estimators on mono-component signals . . . . . . . . . . . . . . . . . . .

2.3 Correlation coefficients between the estimated and referenced instantaneous frequency resulted from various time-frequency estimators on multi-component signals . . . . . . . . . . . . . . . . . . .

2.4 Correlation coefficients between the estimated and referenced instantaneous frequency resulted from various time-frequency estimators on mono-component signals under the various levels of white Gaussian and white uniform noise .

2.5 Correlation coefficients between the estimated and referenced instantaneous frequency resulted from various time-frequency estimators on multi-component signals under the various levels of white Gaussian and white uniform noise

3.1 Polynomial coefficients of the referenced and the synthesized chirp signals . .

3.2 Correlation coefficient between the synthesized and referenced signals in the cases of linear chirp and parabolic chirp from Figures (3.2(a)) and (3.3(a)) under different noise levels and noise types . . . . . . . . . . .

4.1 Polynomial coefficients of the referenced and the synthesized chirp signal component from the multi-component signal $\ldots \ldots \ldots \ldots \ldots$

5.1 Correlation coefficient between the synthesized and referenced chirp in continuous and quantized forms for various length of the signals . . . . . . . .

5.2 Correlation coefficient between the synthesized and referenced chirp in continuous and quantized forms with and without the instantaneous frequency estimation process $\ldots \ldots \ldots \ldots \ldots \ldots \ldots \ldots \ldots$ 


\section{Chapter 1}

\section{Introduction}

Time-Frequency Distribution (TFD) has been proven to be a very powerful tool in signal analysis and signal synthesis $[1,2,3]$. TFD was created as a replacement for the Fourier Transform to analyze non-stationary signal. The standard Fourier analysis is used mainly for stationary signal whose frequency content does not change with time. To use Fourier analysis on non-stationary signal, the signal has to be segmented by a short-time window. During this short period of time, the signal is considered stationary, therefore the Fourier Transform can be used. The technique of taking the Fourier Transform on short windows of a signal is called the Short Time Fourier Transform (STFT). However, in many applications, the involved signals have their frequency content changing so fast that even after windowing, the signal can't be considered stationary and therefore, taking the Fourier Transform of these windowed signals will result in invalid information of the spectral content. To be able to study the time-varying spectra of the analysis signal, a joint TFD representing the energy density of the signal in time and in frequency simultaneously was introduced $[4,1,2]$. It involves mapping a one-dimensional signal into two-dimensional function of time and frequency. The distribution is obtained by calculating the Fourier Transform of the ambiguity function of the signal of interest. TFD provides information on the characteristics of the signal in question with respect to time. Using a joint TFD to represent a time-varying spectrum is advantageous. It utilizes the mathematical properties of a distribution to calculate several parameters such as the sum of the energy of the signal at a particular frequency, the time 
slot or time instant at which a frequency component occurs (instantaneous frequency), and the average frequency at a time instant and its local spread.

The most important application of TFD is the estimation of Instantaneous Frequency (IF) which monitors the frequency change of a signal over time. It has applications on timevarying signals in the areas such as analysis, modification and synthesis. In signal analysis, the TFD is used to provide an idea on how the spectral content of the signal evolves with time. The TFD is also used in signal design, time-frequency filtering, time-frequency noise suppression and in signal synthesis. The most desirable properties of TFD are high joint time-frequency resolution and excellent cross-term removal. Cross-term or interference-term is the component located between two signal components in the TFD. The interference term does not belong to the signal therefore its presence will lead to an incorrect interpretation of the signal properties, and thus, it should be removed.

The earliest TFD was derived from the STFT and called the Spectrogram (SP) $[4,2,5]$. It was used widely in processing of a variety of signals such as the speech signal. The Spectrogram has very high ability in interference term removal. The draw back of the Spectrogram, however, is its low joint time-frequency resolution because of the fixed length of the window used. Due to the uncertainty principle, high resolution in time and in frequency can't be achieved at the same time. The successor of the Spectrogram, the Wigner-Ville Distribution (WVD) $[1,2,4,6]$, has excellent joint time-frequency resolution compared to the Spectrogram. WVD also has two drawbacks that make it become limited in practical applications. The first draw back is the presence of interference terms and the second drawback is that WVD sometimes contains negative values. These problems create difficulties in interpreting the WVD. Besides the Spectrogram and WVD, there are many other TFDs. Most of them belong to a general class called the Cohen's class. Their properties vary, and depending on the applications at hand, their performances are also different. While some TFDs are excellent at removing interference term, their joint time-frequency resolution may be low and their performance may be reduced under the effect of noise. For many researchers, choosing the right TFD among the available TFDs for the particular application is always a difficult 
decision to make. Currently, there are many techniques to calculate the TFD of a signal. Most of the available literature has been focusing on the individual TFD's performance and properties, and very little work has been done on the comparison among the TFDs.

Time-frequency signal synthesis is the process of reconstructing the 1D time domain signal from the TFD. In TFD-based signal design and signal synthesis applications, the TFD which is used to generate the time domain signal is usually modified. The modification often results in an invalid TFD whose inverse Fourier Transform is not the original signal of interest. Therefore, some special algorithms are needed to estimate the time domain signal from the TFD.

The history of time-frequency signal synthesis started when the time domain signal was first synthesized from the STFT and its modified version $[3,7]$. Later on, a variety of radar signals were synthesized successfully from the ambiguity function which is a complexvalued bilinear signal transformation for combined time and frequency shift $[4,8]$. The significant work of signal synthesis from the bilinear TFD was first done by Bartels and Parks in [3]. In their work, the signal is synthesized from the WVD using least squares approximation. The other available algorithms for synthesizing time domain signal from the WVD are the outer-product approximation and basis function approximation proposed by Yu and Cheng [9]. Krattenthaler and Hlawatsch extended the work in [3] and developed algorithms to synthesize the signal from the discrete WVD and the smoothed versions of the WVD $[10,11,12,13,14,15]$. Jeong and Williams later proposed the method of extended discrete time Wigner distribution (EDTWD) [16] to overcome the drawback of Bartels and Parks' method and it was used in spread spectrum communication to mitigate the effect of jamming signals $[17,18,19,20,21]$.

The important signal synthesis algorithms mentioned previously are based on least squares approximation. These algorithms have very high computational complexity which leads to their limited use in practical applications. The alternate way to synthesize a signal is to parameterize the signal using Discrete Polynomial Phase Transform (DPPT). In the DPPTbased approach, the phase of the signal is modeled as a polynomial, and the coefficients 
of this polynomial are determined by the examination of the spectral peak of the ambiguity function. The computational complexity of the DPPT-based signal synthesis algorithm is low compared to that of the methods based on least squares approximation. The only drawback of this algorithm is that it can be used only on mono-component signal. And in fact, many practical signals are of multi-component nature. The best way to overcome the drawback of the DPPT on multi-component signal is to use it in combination with the TFD. The role of the TFD is to isolate the mono-components so the DPPT can be applied on the isolated components to estimate the polynomial phase coefficients. This is the main motivation of this thesis and it is also the reason for studying the localization of TFD as well as their ability to generate faithful instantaneous frequency.

Signal synthesis has been used in the analysis and processing of time-varying signals. The applications include time-varying filtering, signal separation, time-frequency noise reduction, mitigation of jamming signal in radar applications, error correction algorithm, and biomedicine (e.g. electroencephalogram (EEG) seizure analysis in newborns [22]). The synthesis algorithms are also used to estimate the instantaneous frequency of a signal as well as to design a signal whose TFD satisfies some desirable properties. One of the most common applications of signal synthesis is the synthesis and detection of chirp signal which has applications in many areas.

Chirp signals are present ubiquitously in many areas of science and engineering. Chirps are identified in natural signals such as animal sounds (birds, frogs, whales and bats), whistling sound, as well as in man made systems such as in radar, sonar, telecommunications, and acoustics. For example, in radar applications, chirp signals are used to analyze the trajectories of moving objects. Due to its inherent ability to reject interference, linear frequency modulated signals or chirp signals are also used widely in spread spectrum communications. Chirps are also involved in biomedicine applications such as in the study of EEG and electromyogram (EMG) data.

Recently, the boom in technology makes it easier for digital contents to be copied and reproduced illegally in large quantities beyond the control of content providers. Annually, 
the loss due to US intellectual property piracy is estimated at $25-30$ billion dollars [23]. This huge amount dose not cover the lost revenue due to piracy over the Internet. The estimated number of movies that are available for uploading and downloading is 500,000 on a daily basis. With the increase in bandwidth of broadband connection, a whole movie can be downloaded in just 40 minutes. And with the next generation of the Internet, the time to download a movie will decrease to about 45 seconds [24]. The current situation results in the need for a new technology that can work against piracy problem. The technology should be used as a tool to prove the content's origin, protect the copyrights and prevent illegal use. Digital watermarking is a technique to embed a message into digital contents such as multimedia. Depending on the application at hand, the message can be visible/audible or invisible/inaudible. The embedded message is called the watermark and it is recovered during the extraction process. In many current watermarking technologies, the embedding and extracting processes are performed in either spatial, or spectral domain. Few watermark applications utilize the joint time-frequency domain as the processing domain. The watermarking of audio signals and images in $[25,26]$ uses the time-frequency domain as the processing domain to extract the watermark message. In those works, the chirp message is embedded in the signals and then detected at the receiver based on its frequency change rate. The extraction process successfully detects the linear chirp embedded as watermark by applying the Hough-Radon Transform (HRT) based detection algorithm on the TFD of the received chirp. HRT-based chirp detector is a well-developed and only technique for detecting directional lines in a two dimensional image, but it requires a high degree of computational complexity because the involved calculation method is very similar to that of the maximum likelihood technique.

In this thesis, performance of the common TFDs is compared under various conditions. The criteria for the evaluation are the joint time-frequency resolution, the ability to suppress interference terms of the TFD, and how close the Instantaneous Mean Frequency (IMF) estimated from the TFDs is compared with the referenced IMF. The simulation is done for different types of signals under various noise levels and noise types. The candidate TFD 


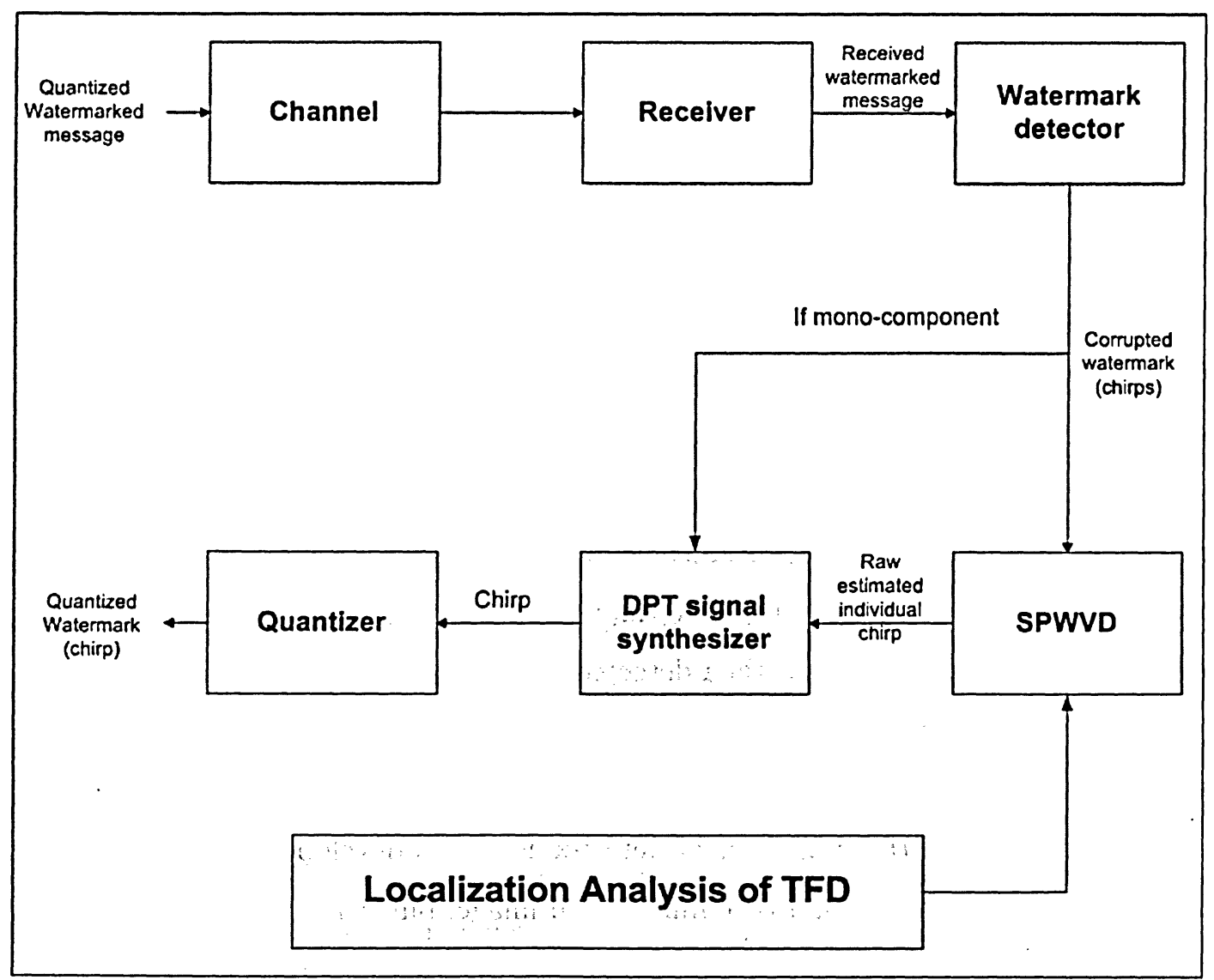

Figure 1.1: The block diagram of the proposed system. 
which performs well under all conditions is then used as the processing TFD in the signal synthesis application. A novel signal synthesis algorithm is also proposed in this thesis. The algorithm combines the DPPT with the candidate TFD to synthesize linear and quadratic time domain chirp signals. The technique is then applied in the detection of the watermark message in multimedia watermark application as an alternative method for the HRT-based detection method. The proposed DPPT-based detection has lower computational complexity compared to HRT-based approach so it can be used in more practical applications. Figure 1.1 is an overview of the described system.

The rest of the thesis is divided into 5 chapters. Chapter 2 is the chapter on the localization analysis of the TFDs. In this chapter, general theory on the TFD is discussed, then the concept of the TFD kernel is introduced and used in the analysis of the TFD behaviors. The simulation in the last section is done on different types of signals under various noise conditions. Chapter 3 provides an overview of time-frequency signal synthesis. DPPT-based and HRT-based signal synthesis methods are considered. The last section of this chapter is for the simulation of linear and parabolic chirp signals using a DPPT-based signal synthesis method. In Chapter 4, the chirp synthesis and detection method are proposed. The new method applies the DPPT algorithm in parallel with the Smoothed Pseudo Wigner-Ville Distribution (SPWVD) to synthesize and detect the chirp signal. The detection scheme is designed in a way that it can be used on mono-component signals and expanded to be used on multi-component signals. In Chapter 5, the proposed detection technique in the previous chapter is used in the multimedia security applications. Finally, Chapter 6 presents the conclusions. This chapter discusses the simulation result, the contribution of this thesis work as well as the possible future work relating to the technique. 


\section{Chapter 2}

\section{Time-Frequency Localization Analysis}

\subsection{General Theory}

The Fourier Transform has been used as a standard tool for many years to analyze the spectral characteristics of signals and it is still a powerful method for that purpose. However, the Fourier Transform can be applied only on stationary signals and it does not provide information on when the spectral components of the signal occur and how they change with time. Since the majority of signals in real-life are of non-stationary nature, a tool is needed to monitor the instantaneous frequency of a signal over time. Instantaneous frequency is an important parameter in the study of non-stationary signals and it has been used widely by researchers in many applications and areas such as radar, sonar, telecommunications, and biomedicine.

Traditionally, the instantaneous frequency $f_{m}(t)$ is obtained by taking the first derivative of the phase of the analytic signal with respect to time:

$$
f_{m}(t)=\frac{1}{2 \pi} \frac{d \phi(t)}{d t},
$$

where $\phi(t)$ is the phase of the signal. This method of calculating instantaneous frequency poses some difficulties because the phase of the signal is not always available and the derivative of the phase of the signal may take negative values thus misleading the interpretation of instantaneous frequency. Another way to estimate the instantaneous frequency of a signal is to take the first central moment along each time slice of the TFD. TFD has been used 
widely as an analysis tool for the study of non-stationary signals. It involves mapping a one dimensional signal $x(t)$ into a two dimensional function $T F D_{x}(t, f)$, which provides the information on spectral characteristics of the signal with respect to time. Time-frequency representations are classified into two main groups: linear and quadratic. One example of linear time-frequency representation is the STFT. In the STFT, a non-stationary signal is divided into short window segments in time, so that each segment can be considered stationary. Fourier Transform is then calculated for each segment to obtain spectral information of the time instants corresponding to the particular segments.

$$
\operatorname{STFT}_{x}(t, f)=\int_{-\infty}^{+\infty} x(\tau) h(\tau-t) e^{-j 2 \pi f \tau} d \tau,
$$

where $\tau$ is the running time, $x(t)$ and $h(t)$ are the signal and analysis windows respectively. The Spectrogram is derived from the STFT by squaring its amplitude. The main drawback of the STFT and the Spectrogram is the trade-off between time and frequency resolution due to the choice of the fixed window length used. Due to the Heisenberg inequality (uncertainty principle) [27], high resolution in time and in frequency can't be achieved at the same time. A narrow running analysis window results in good time resolution (better localization in time) and a wide frequency bandwidth (low frequency resolution). On the other hand, a good frequency resolution will require a longer analysis windows which reduces the time resolution. Due to this time-frequency localization disadvantage of the Spectrogram and the STFT, a new procedure was needed for time-frequency representation. Quadratic (or bilinear) time-frequency representation such as WVD uses energy distribution of the signal over time and frequency to represent the temporal and spectral information. Given a signal $x(t)$, its WVD is calculated as:

$$
W V_{x}(t, f)=\int_{-\infty}^{+\infty} x\left(t+\frac{1}{2} \tau\right) x^{*}\left(t-\frac{1}{2} \tau\right) e^{-j 2 \pi f \tau} d \tau
$$

WVD has been studied extensively by many researchers $[1,2,4,6]$, because the distribution satisfies several mathematical properties which are desirable for time-frequency representation. The distribution is real and having high joint time-frequency resolution in addition to satisfaction of the time and frequency energy marginals which are obtained by 
integrating the distribution in time or frequency directions. However, the WVD is not always positive and contains interference terms when it is applied on multi-component signals. The drawback of the WVD is the motivation for introduction to other TFDs such as Pseudo Wigner-Ville Distribution (PWVD), SPWVD, cone kernel distribution. There are a large number of possible TFDs and they are classified based on the desired properties such as interference term removal and joint time-frequency resolution. There is always a trade off between resolution and interference term suppression. The removal of interference terms (smoothing) also takes away some of the signal energy and reduces the joint time-frequency resolution. When it comes to evaluation of a TFD, besides the factors such as accuracy of the instantaneous frequency estimation, high resolution in joint time-frequency domain, ability to suppress interference terms, one should also consider the effects of noise on the TFD's performance. But first, let us have a brief review on some basic theory on TFD.

\subsection{Cohen's Class of TFD}

The general expression for a TFD $T_{x}(t, f)$ was derived by Leon Cohen [4]:

$$
T_{x}(t, f)=\iiint x\left(u-\frac{1}{2} \tau\right) x^{*} \cdot\left(u+\frac{1}{2} \tau\right) \Psi_{T}(\tau, \nu) e^{-j 2 \pi f \tau} d \nu d u d \tau
$$

where $\Psi_{T}(\tau, \nu)$ is called the kernel of the distribution. Different TFDs have different properties depending on the kernel function.

\subsubsection{Desired Properties of the TFD}

\section{Energy conservation}

Integration of the distribution along frequency and time axes results in the total energy of the signal $x(t)$ :

$$
\int T_{x}(t, f) d f d t=1
$$

The above condition is equivalent to having $\Psi_{T}(0,0)=1$.

2. Marginals properties (instantaneous energy and spectrum)

The instantaneous power $|x(t)|^{2}$ and the energy density spectrum $|x(f)|^{2}$ are calcu- 
lated by integrating the distribution along frequency and time directions respectively (marginals of the distribution).

$$
\begin{aligned}
& \int T_{x}(t, f) d f=|x(t)|^{2}, \\
& \int T_{x}(t, f) d t=|x(f)|^{2} .
\end{aligned}
$$

This condition is equivalent to having $\Psi_{T}(\nu, 0)=1$ and $\Psi_{T}(0, \tau)=1$ respectively.

\section{Reality}

The distribution should be real to avoid interpretive problem. A necessary and sufficient condition for a distribution to be real is:

$$
\Psi_{T}(\tau, \nu)=\Psi_{T}^{*}(-\tau,-\nu) .
$$

4. Time and frequency shift (also called translation covariance)

A shift in time or a shift in frequency of the signal will result in the corresponding shift of the joint TFD

$$
\begin{aligned}
x\left(t+t_{0}\right) & \Rightarrow T_{x}\left(t+t_{0}, f\right), \\
x(t) e^{-j 2 \pi f_{0} t} & \Rightarrow T_{x}\left(t, f+f_{0}\right) .
\end{aligned}
$$

5. Positivity

The distribution should be positive:

$$
T_{x}(t, f)>0 .
$$

6. Instantaneous frequency

The instantaneous frequency of the signal under analysis can be determined by taking the derivative of the phase of the corresponding analytic signal as in Equation (2.1). It can also be calculated by taking the first conditional moment in time of the TFD:

$$
f_{m}(t)=\frac{\int_{-\infty}^{+\infty} f T_{x}(t, f) d f}{\int_{-\infty}^{+\infty} T_{x}(t, f) d f} .
$$

Based on this method of instantaneous frequency calculation, instantaneous frequency can be defined more precisely as the mean frequency of the signal at time $t$. Taking 
the second conditional moment in time of the TFD may provide information on the spread or broadness of the frequency component at that instant $t$. This parameter is called the instantaneous bandwidth $B(t)$ :

$$
B^{2}(t)=\frac{\int_{-\infty}^{+\infty} f^{2} T_{x}(t, f) d f}{\int_{-\infty}^{+\infty} T_{x}(t, f) d f}-f_{m}^{2}(t)
$$

\section{Group delay}

Similar to the concept of instantaneous frequency, group delay can be interpreted as the mean time of the signal at a particular frequency. Group delay is calculated by taking the first conditional moment in frequency. Due to this similarity, group delay and instantaneous frequency are called dual pair.

$$
t_{m}(f)=\frac{\int_{-\infty}^{+\infty} t T_{x}(t, f) d t}{\int_{-\infty}^{+\infty} T_{x}(t, f) d t}
$$

\subsubsection{Bi-linearity and Interference Term}

From the general expression of Cohen's class TFD in Equation (2.4), it is obvious that the TFD depends on the kernel function $\Psi_{T}(\tau, \nu)$ and the signal energy term. The kernel can be a function of $\nu, \tau$, and of the signal itself. If the kernel does not depend on the signal, the kernel is said to be signal-independent and the corresponding TFD is called a bilinear TFD. Unlike linear TFD such as the STFT, TFD of a signal consisting of several mono-component terms is not the sum of the TFD of the mono-component. Given signal $x(t)$ consisting of two components $x_{1}(t)$ and $x_{2}(t)$ :

$$
x(t)=c_{1} x_{1}(t)+c_{2} x_{2}(t) .
$$

By superposition principle, the Cohen's class TFD of the signal is expressed as [2]:

$$
T_{x}(t, f)=\left|c_{1}\right|^{2} T_{x_{1}}(t, f)+\left|c_{2}\right|^{2} T_{x_{2}}(t, f)+c_{1} c_{2}^{*} T_{x_{1} x_{2}}(t, f)+c_{2} c_{1}^{*} T_{x_{2} x_{1}}(t, f)
$$

in which the first two terms are the TFD of the signal components and called the signal terms or auto terms. The last two terms in the equation are the cross TFD between the 
signal components, which are also called interference terms:

$$
T_{x_{i}, x_{j}}(t, f)=\int_{-\infty}^{+\infty} x_{i}\left(t+\frac{1}{2} \tau\right) x_{j}^{*}\left(t-\frac{1}{2} \tau\right) e^{-j 2 \pi f \tau} d \tau
$$

For a signal consisting of $N$ components, the total number of interference terms is $N(N-1) / 2$. On a time-frequency plane, the interference terms appear mid way between the positions of the auto terms. These interference terms oscillate in the direction perpendicular to the lines joining the auto terms. The presence of interference terms makes it extremely difficult to interpret and to study the TFD of the signal and therefore they are components to be removed.

\subsubsection{The Analytic Signal and Spectral Aliasing}

Given a real signal $x(t)=a_{0} \cos (\phi(t))$, where $\phi(t)$ is the instantaneous phase of the signal. The instantaneous frequency of $x(t)$ is determined by taking the derivative of the phase of the analytic form of $x(t)$ as in Equation (2.1). The analytic form $z(t)$ is calculated using the Hilbert Transform:

$$
z(t)=x(t)+j \mathcal{H}[x(t)]
$$

Using the analytic signal has advantage in the calculation of the instantaneous frequency. From Equation (2.9) in the previous part, the first moment in time of a TFD equals the instantaneous frequency of the signal. In fact, the equality holds true only when the signal involved is an analytic signal. In addition, using an analytic signal can avoid spectral aliasing in the time-frequency plane. The spectrum of a real valued signal $x(t)$ has both positive and negative components. These components interact with each other and result in the interference terms or spectral aliasing between them in the time-frequency domain. When taking the Hilbert Transform, the negative component is removed, therefore aliasing is avoided. In the case of the WVD, the spectral aliasing could be suppressed by sampling the input signal at twice the Nyquist sampling rate. But if an analytic signal is used, the signal can be sampled at the Nyquist rate without the occurrence of spectral interference. 


\subsection{The Ambiguity Domain and the Trade-off Between the Joint Time-Frequency Resolution and the Abil- ity To Remove Interference Terms}

All TFDs which belong to Cohen's class can be represented as a two dimensional convolution in the equation below $[2,28]$ :

$$
T_{x}(t, f)=\int_{t^{\prime}} \int_{f^{\prime}} \psi_{T}\left(t-t^{\prime}, f-f^{\prime}\right) W_{x}\left(t^{\prime}, f^{\prime}\right) d t^{\prime} d f^{\prime},
$$

where $W_{x}(t, f)$ is the WVD of the signal $x(t)$ and $\psi_{T}(t, f)$ is the real value smoothing kernel of the distribution.

The above convolution in the time-frequency domain is equivalent to multiplication in the ambiguity domain $(\tau, \nu)$ :

$$
T_{x}(\tau, \nu)=\Psi_{T}(\tau, \nu) A_{x}(\tau, \nu)
$$

where $\Psi_{T}(\tau, \nu)$ is calculated as the $2 \mathrm{D}$ Fourier Transform of the real value kernel $\psi_{T}(t, f)$ :

$$
\Psi_{T}(\tau, \nu)=\int_{t} \int_{f} \psi_{T}(t, f) e^{-j 2 \pi(\nu t-\tau f)} d t d f
$$

and $A_{x}(\tau, \nu)$ is the ambiguity function calculated by taking the Fourier Transform of the Wigner-Ville $W_{x}(t, f)$ :

$$
A_{x}(\tau, \nu)=\int_{t} x\left(t+\frac{\tau}{2}\right) x^{*}\left(t-\frac{\tau}{2}\right) e^{-j 2 \pi \nu t} d t
$$

In the ambiguity domain, the signal auto term are centered at the origin while the interference terms are located away from the origin. The kernel acts as a low pass filter on the Wigner distribution of the signal, smooths out the interference terms and retains the auto terms. The ambiguity domain makes it easier and more convenient to design and study the properties of a smoothing kernel. For example, depending on the properties of the signal of interest, one can adjust the shape of the kernel to make it satisfy the marginal properties as discussed in the previous section by setting $\Psi_{T}(\nu, 0)=1$ and $\Psi_{T}(0, \tau)=1$, or make the kernels have the ability of removing the interference terms effectively. In addition, 
the comparison between the TFD in Cohen's class will become easier by visualization of the smoothing kernels in the ambiguity domain. This is similar to the preference of designing a one-dimensional filter in the frequency domain instead of the time domain in regular filter applications. Therefore, in order to study the properties of a time-frequency estimator, it is more advantageous to examine the shape of the corresponding smoothing kernel in the ambiguity domain $[2,28]$.

Figure 2.1 shows the location of the signal auto terms and the interference terms in real value domain $(t, f)$ and ambiguity domain $(\tau, \nu)$ for two signal points $\left(t_{1}, f_{1}\right)$ and $\left(t_{2}, f_{2}\right)$. In the real-value time-frequency domain (Fig. 2.1(a)), the interference term term is located at the midpoint between the two signal auto terms, and it oscillates in the direction perpendicular to the line between the auto terms. In the ambiguity domain (Fig. 2.1(b)), the auto terms are located at the origin and the interference terms are located away from the origin at $\left(\tau_{1,2}, \nu_{1,2}\right)$ and $\left(-\tau_{1,2},-\nu_{1,2}\right)$ where $\tau_{1,2}=\left(\tau_{1}-\tau_{2}\right)$ and $\nu_{1,2}=\left(\nu_{1}-\nu_{2}\right)$ [28].

Ideally, the value of the kernel low pass filter $\Psi_{T}(\tau, \nu)$ should be one in the auto term region and zero in the interference term region, if the kernel is too narrow, suppression of the interference term also takes away some of the auto term energy leading to smearing of the signal components in the TFD. On the other hand, if the kernel shape is too broad, it can reserve the auto term energy but can't suppress all the interference terms. This reason explains why a fixed kernel design (not adaptive) can't work properly for any signal types. High joint time-frequency resolution can't be achieved at the same time with good interference suppression. Figure 2.2 shows the shape of the kernel function (ambiguity domain) of different time-frequency estimators. In the $\mathrm{WVD}_{T} \Psi_{T}(\tau, \nu)=1 \forall \tau, \nu$, the kernel is an all pass filter, no interference term suppression is allowed and the auto term energy is reserved. Therefore the WVD has very high time-frequency resolution at the expense of the presence of interference terms. Behaviors of the above smoothing kernel will be discussed in more detail in later part of the thesis.

It is worth mentioning that there is a relation or constraint between the kernel function and the number of requirements the TFD satisfies. Strictly following the requirements would 


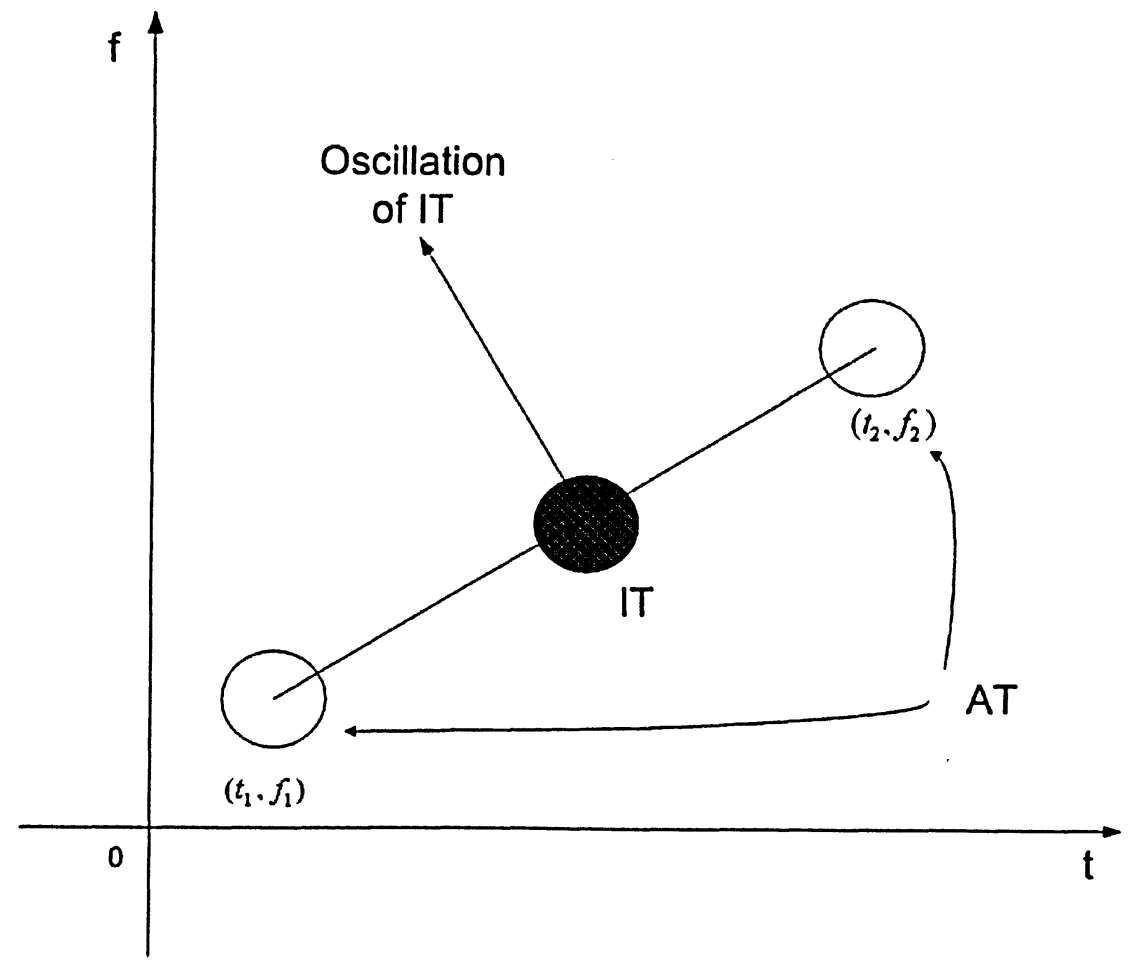

(a) time-frequency domain

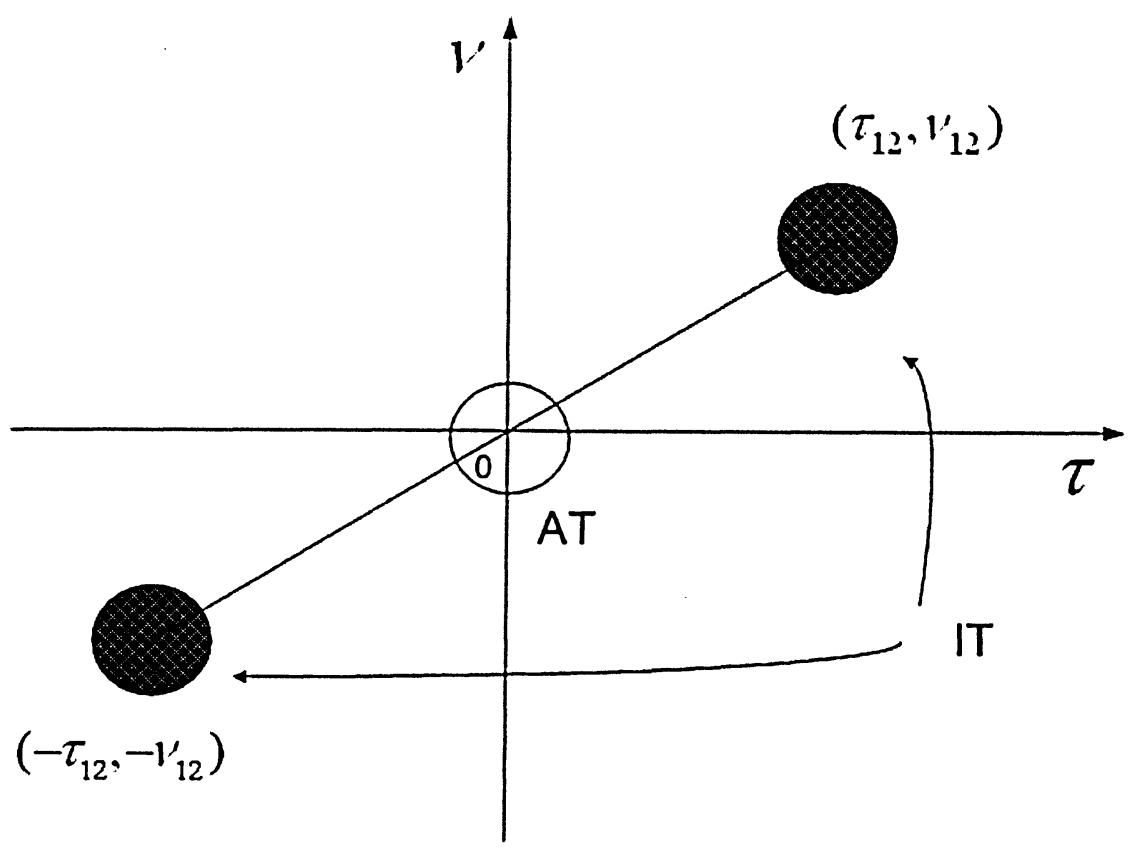

(b) Ambiguity domain

Figure 2.1: Location of interference terms and auto terms
17 


\begin{tabular}{|c|c|c|}
\hline Distribution & Kernel $\varphi_{T}(t, \tau)$ & Kernel $\Psi_{T}(\tau, \nu)$ \\
\hline WVD & $\delta(t)$ & 1 \\
\hline PWVD & $\delta(t) h\left(\frac{\tau}{2}\right) h^{*}\left(-\frac{\tau}{2}\right)$ & $h\left(\frac{\tau}{2}\right) h^{*}\left(-\frac{\tau}{2}\right)$ \\
\hline SPWVD & $g(t) h\left(\frac{\tau}{2}\right) h^{*}\left(-\frac{\tau}{2}\right)$ & $h\left(\frac{\tau}{2}\right) h^{*}\left(-\frac{\tau}{2}\right) G(\nu)$ \\
\hline SP & $\gamma\left(-t-\frac{\tau}{2}\right) \gamma^{*}\left(-t+\frac{\tau}{2}\right)$ & $A \gamma(-\tau,-\nu)$ \\
\hline CW & $\sqrt{\frac{\sigma}{4 \pi}} \frac{1}{|\tau|} \exp \left[-\frac{\sigma}{4}\left(\frac{t}{4}\right)^{2}\right]$ & $\exp \left[-\frac{(2 \pi \tau \nu)^{2}}{\sigma}\right]$ \\
\hline
\end{tabular}

Table 2.1: Smoothing kernels of the common TFDs

create some limitation on interference term suppression. One of the important requirements is the marginal property which states that (in ambiguity domain) $\Psi_{T}(0, \nu)=1 \forall \nu$ and $\Psi_{T}(\tau, 0)=1 \forall \tau$. The Choi-Williams distribution (CWD) satisfies this condition while the SPWVD and the PWVD do not. This results in the presence of interference terms in the CWD if there are more than one signal component at the same time or same frequency instant. This phenomenon is not present in the SPWVD.

\subsection{Smoothing Kernels of the Popular TFDs}

Table 2.1 lists the smoothing kernels of several estimators in $(t, \tau)$ domain and $(\tau, \nu)$ ambiguity domain [2].

\subsubsection{The Spectrogram}

The kernel of the Spectrogram:

$$
\varphi_{T}(t, \tau)=\gamma\left(-t-\frac{\tau}{2}\right) \gamma^{*}\left(-t+\frac{\tau}{2}\right)
$$

is the WVD of the running window $\gamma(t)$. Smoothing is done extensively to remove interference terms, therefore the interference terms will only be present if the signal terms overlap $[2,29]$. This effect can be easily seen from Figure 2.2. The passing region of the Spectrogram is very narrow that it effectively removes all interference terms. However, the Spectrogram suffers from a trade off between time and frequency resolution. If a short window is used, 


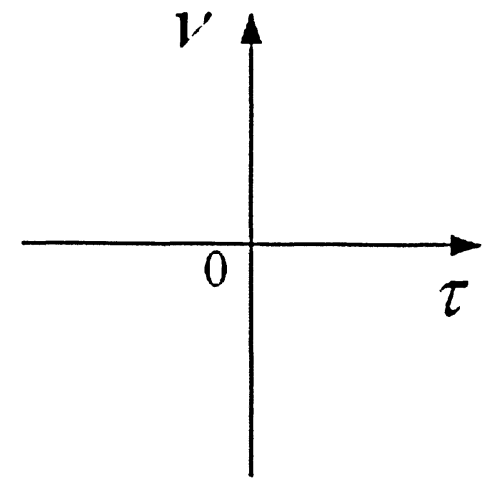

(a)

$\ddot{\bullet}$

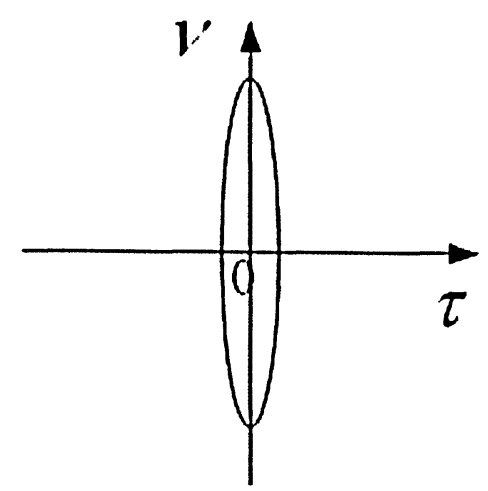

(d)

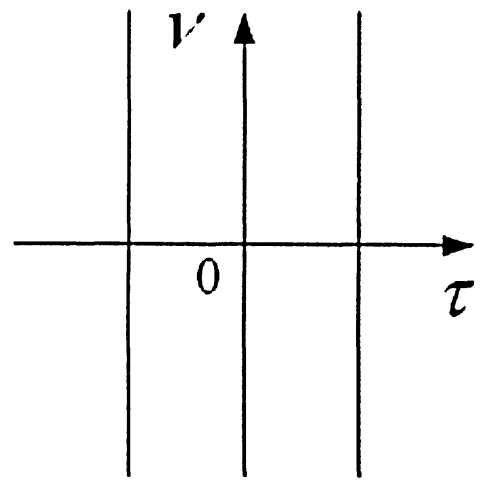

(b)

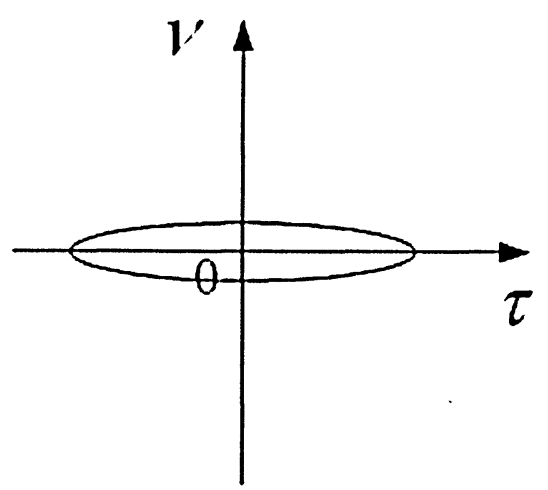

(e)

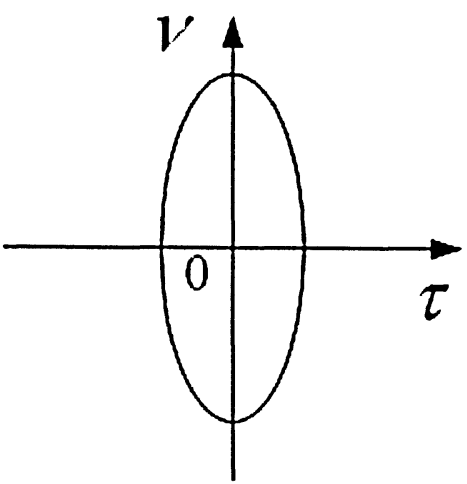

(c)

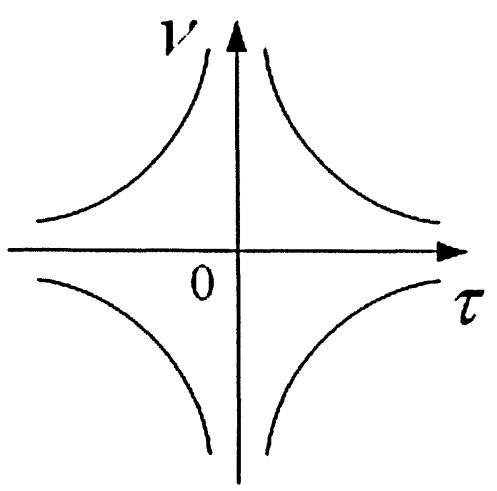

(f)

Figure 2.2: The shape of different TFDs kernel: a)WVD, (b)PWVD, (c) SPWVD, (d) Spectrogram (long window), (e) Spectrogram(short window), and (f) CWD 
smoothing function will be narrow in time and wide in frequency leading to good resolution in time and bad resolution in frequency and vice versa. The TFD plot of the Spectrogram has no interference terms but very low time-frequency resolution because time and frequency smoothing can't be controlled separately as in the SPWVD.

\subsubsection{WVD, PWVD and SPWVD}

In the WVD, the kernel is always one, therefore no smoothing is made between the regions of the ambiguity domain. The SPWVD, on the other hand, has more progressive and independent smoothing control both in time and frequency. The SPWVD's advanced performance can be contributed to its smoothing kernel design. The kernel of the SPWVD and the PWVD in time-frequency domain has the form:

$$
\psi_{T}(t, f)=g(t) H(f)
$$

where $g(t)$ is the time-smoothing window and $h(t)$ is the running analysis window having frequency-smoothing effect. Taking Fourier Transform of $\psi_{T}(t, f)=g(t) H(f)$ we obtain the smoothing kernel in the ambiguity domain (note that $t \stackrel{F}{\rightarrow} \nu$ and $f \stackrel{F}{\rightarrow} \tau$ ):

$$
\begin{aligned}
\Psi_{T}(\tau, \nu) & =H(\tau) G(\nu) \\
& =h\left(\frac{\tau}{2}\right) h^{*}\left(-\frac{\tau}{2}\right) G(\nu) .
\end{aligned}
$$

In the PWVD, $g(t)=\delta(t)$ leads to $G(\nu)=1$, therefore no smoothing is done to remove the interference term oscillating in time direction, smoothing is only possible for oscillation in frequency direction. Since the smoothing of the SPWVD is done in both time and frequency direction, most of its interference terms are attenuated. Smoothing in time and frequency can be adjusted separably with abundant choices of the windows $g(t)$ and $h(t)$. The amount of smoothing in time and frequency increases as the length of the window $g(t)$ increases and length of the window $h(t)$ decreases respectively. Although smoothing of interference term also takes away the auto terms and reduces joint time-frequency resolution, the SPWVD is still more localized than the Spectrogram and does not suffer much from the time-frequency resolution trade-off. According to [2, 28], a SPWVD separable smoothing kernel has the 


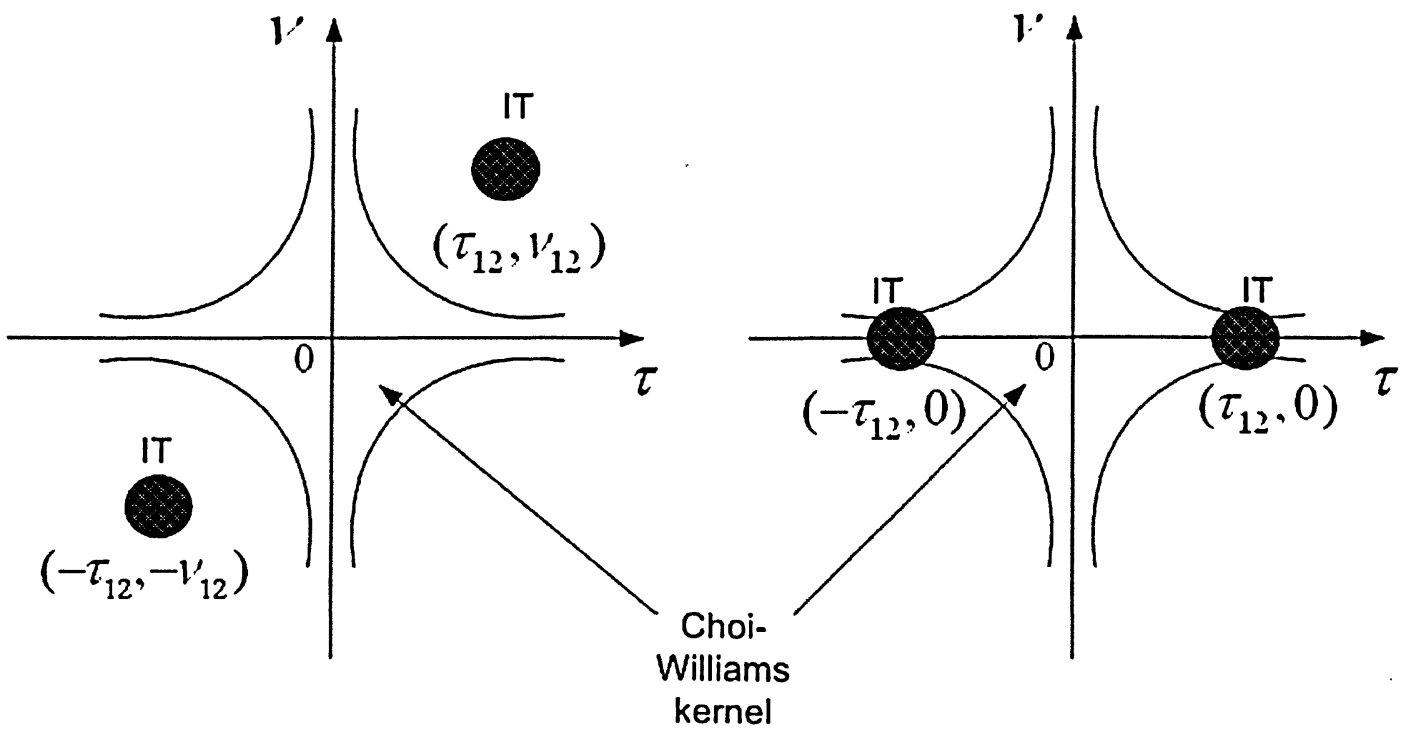

Figure 2.3: CWD kernel

shape of a Gaussian function and its ability to suppress interference term does not depend much on signal types as the CWD kernel does. In the CWD, independent control of time and frequency smoothing is not possible. This limitation as well as the requirement on marginal property reduce the distribution's ability to remove interference terms and make it less versatile than the SPWVD.

\subsubsection{CWD}

Unlike the SPWVD, independent control of time and frequency smoothing is not possible in the CWD. The kernel of the CWD has a cross shape in ambiguity domain. Since $\Psi_{T}(\tau, \nu)=$ $\exp \left[-\frac{(2 \pi \tau \nu)^{2}}{\sigma}\right], \Psi_{T}(\tau, 0)=1 \forall \tau$ and $\Psi_{T}(0, \nu)=1 \forall \nu$, the marginal property is always satisfied. This causes a limitation on removal of interference terms if the signal components occur at the same time or frequency instant. Let's say, for example, if the signal terms occur at the same frequency instant (Figure 2.3), $\left(f_{1}-f_{2}\right)=0$, the corresponding interference term will locate on $\tau$-axis. Since $\Psi_{T}(\tau, 0)=1 \forall \tau$, this interference term can't be removed. Parameter $\sigma$ is used to control the smoothing in both frequency and time direction. A large $\sigma$ results in a broader kernel with less ability of removing interference terms. A good value of $\sigma$ can help 
reducing interference term significantly while maintaining auto term energy. If the signal terms do not occur at the same time and frequency instants, optimizing $\sigma$ can result in highly concentrated TFD compared to the SPWVD. However, the cross shape of the kernel also makes this distribution depend heavily on the signal types and hence less versatile compared to the SPWVD. In Figure 2.4, the CWD has better joint time-frequency than the SPWVD but some interference terms are still present between the signal components because these signal components occur at the same frequency instant [2, 28]. Figure 2.4 shows the TFD of some popular time-frequency estimators. The SPWVD is almost free of interference terms but its joint time-frequency resolution is reduced compared to WVD due to the effect of smoothing.

\subsection{Performance Comparison Among The TFD's}

As stated from the previous section, there is always a trade off between resolution and interference term suppression. The removal of interference term (smoothing) also takes away some of the signal energy and reduces the joint time-frequency resolution. When it comes to evaluation of a TFD, besides the factors such as accuracy of instantaneous frequency estimation, high resolution in joint time-frequency domain, ability to suppress interference terms one should also consider the effects of noise on the TFDs performance.

The author has done several simulations to compare the properties of different TFDs on various signals, types and levels of noise. The TFDs involved in the test are the Spectrogram, the WVD, the PWVD, the SPWVD, the CWD, the Page distribution, the pseudo-Page distribution, the Chirplet Transform (CT) and the matching pursuit decomposition based TFD (MPTFD) technique. Among the examined time-frequency representations, only the MPTFD and the Chirplet Transform are adaptive in nature. Chirplet Transform computation is extensive depending on the number of chirps used. The MPTFD has its adaptiveness based on the decomposition algorithm [30,31] and the choice of the dictionary. Both methods can be adjusted to generate a TFD which is clean and interference-term-free but at the expense of heavy computation. They are included in the comparison but the focus of the sim- 


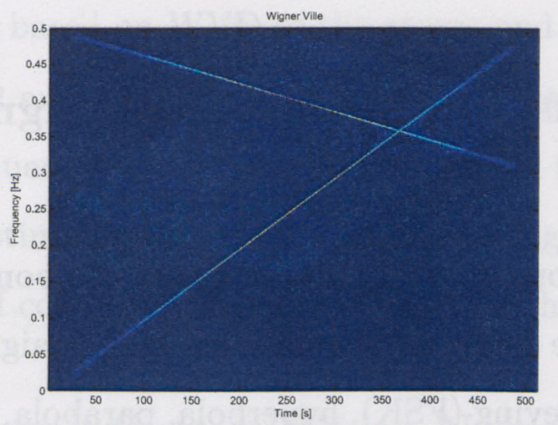

(a) WVD

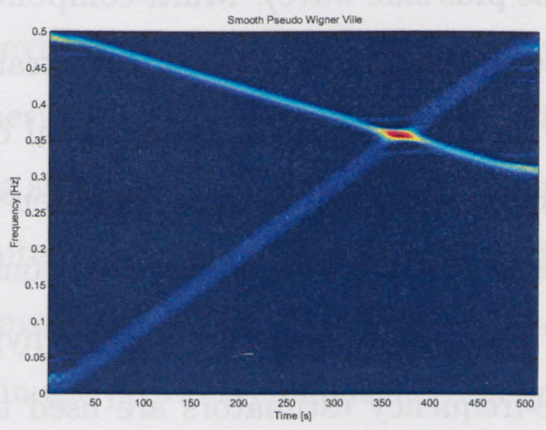

(c) SPWVD

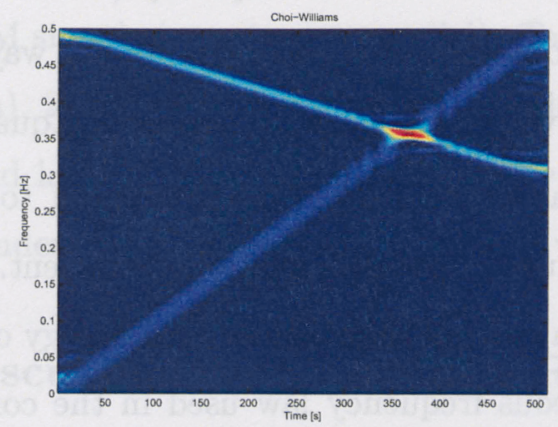

(e) CWD

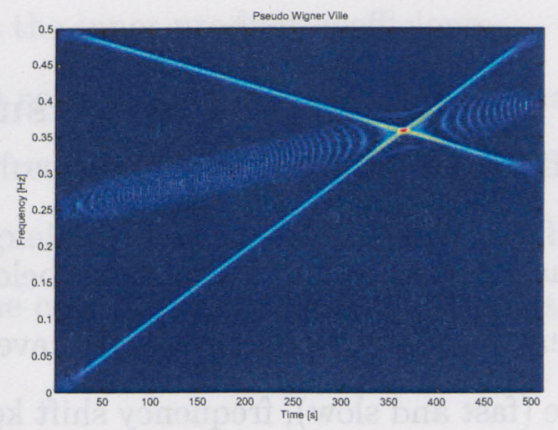

(b) PWVD

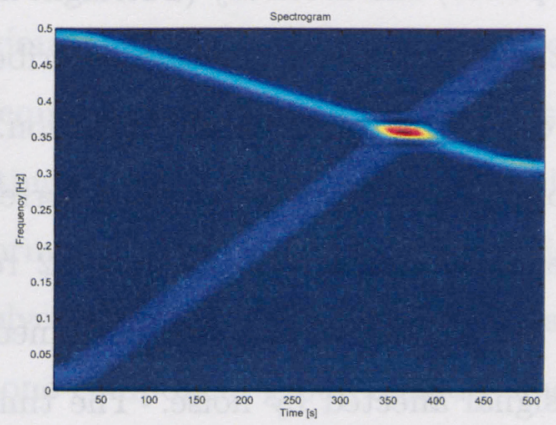

(d) Spectrogram

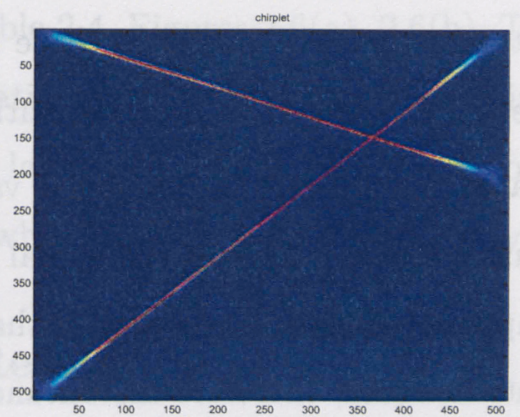

(f) Chirplet transform

Figure 2.4: TFD of several popular estimators 
ulation is on the TFDs with signal-independent kernels since computational efficiency is one of the requirements for the TFD applications in multimedia applications such as information security.

\subsubsection{Mono-component versus Multi-component Signal Simula- tion}

The signals tested in the simulations belong to two groups: mono-component and multicomponent. Mono-component signals have frequency modulation of a straight line (linear), sine wave (fast and slow), frequency shift keying (FSK), hyperbola, parabola, doppler, power law (FM-power) and arbitrary (a straight line plus sine wave). Multi-component signals have their instantaneous frequency laws described in the following cases: two straight lines either crossing each other or in parallel position. In the parallel case, the signal components are either close to each other or far apart corresponding to the distance between them measured in normalized frequency of 0.1 and 0.2 respectively. The other multi-component signals involved are a sine wave above combined but not intersecting with a hyperbola, and a doppler signal affected by noise. The time-frequency estimators are used to calculate the TFD of the test signals and estimate their instantaneous frequency (first moment in time). The results are then compared to those obtained using the traditional way of calculating instantaneous frequency based on derivative of the signal's phase as in Equation (2.1).

Estimated instantaneous frequency values from TFD are compared to the known instantaneous frequency laws of the signal using cross correlation coefficient. In the case of multi-component signals, it is assumed that at any time instant, the energy of the individual components is equal, therefore instantaneous frequency law used in the comparison is the average of the instantaneous frequency of the individual components.

All distributions are computed using the corresponding functions at their default parameter values except the Chirplet Transform and matching pursuit decomposition. In the Chirplet Transform case, unless specified, the number of chirps used to represent a signal is ten, and this is selected on the basis of the dynamics of the signal. The Chirplet Transform 
TFD is the sum of the Wigner-Ville TFD of the individual chirps. In matching pursuit decomposition, the signal of interest is decomposed into Gaussian atoms or time-frequency functions. The number of iterations for signal decomposition is 100. The TFD of the signal is computed based on WVD of the atoms and the inner product coefficients.

Table 2.2 and Figure 2.5(a) show the cross correlation values between estimated instantaneous frequency and known instantaneous frequency laws resulting from different timefrequency estimators on single-component signals. Table 2.3 and Figure 2.5(b) show the same type of correlation coefficients but for the case of multi-component signals.

\subsubsection{Simulation in White Noise}

From the previous simulation result, it is obvious that the overall performance of the SPWVD is superior to that of the other time-frequency estimators for different signal types. The following simulation is intended for evaluating the SPWVD performance under the effect of noise. White Gaussian noise and white uniform noise were added to the test signals at different SNR levels of 0, 1,5 and $10 \mathrm{~dB}$. The involved signals in these tests are the same type of signals used in the previous simulation. The mono-component group includes linear and sine frequency modulated signals. The multi-component group includes the doppler signal and two linear FM signals (crossing or parallel). Table 2.4, Figures 2.6(a), 2.6(b), Table 2.5, and Figures 2.7(a), 2.7(b), 2.8 give the cross correlation value between estimated instantaneous frequency and known instantaneous frequency laws with different instantaneous frequency estimators under the effect of white noise and white uniform noise respectively.

\subsubsection{Discussion on Simulation Result}

Performance of the TFD estimators varies depending on the characteristics of the input signals such as linearity, rate of frequency change, mono or multi-component and the proximity of the frequency components in the signal. When the signal of analysis is a mono component signal, almost all estimated instantaneous frequencies are highly correlated with the corresponding known instantaneous frequency law except estimated instantaneous frequency 
from the Page Distribution. In the case of frequency modulation of a sine wave, increasing frequency rate reduces performance of the Chirplet Transform significantly with the same number of chirplets used. This may be explained as that the chirplet dictionary does not have enough chirplet atoms to represent such a dynamic signal. Matching pursuit cross correlation value is very low in frequency shift keying signal (named as FSK in the table). For mono component signals, estimation of instantaneous frequency by taking derivative of the phase yields highly correlated instantaneous frequency among different signal types.

For multi component signals, the SPWVD scores very high among different signal types. The WVD, the PWVD and the derivative of the signal's phase (d(phase)) become unreliable for estimating instantaneous-mean-frequency because of the effect of interference terms. The Spectrogram, the Chirplet Transform and the matching pursuit decomposition based TFD techniques have stable scores throughout the test. The Chirplet Transform seems to work well only with linear instantaneous frequency modulation. The WVD technique is working only for mono-component signal, its performance degrades when there are more than one frequency components at a time instant especially when the distance between the components is getting closer (linear parallel-close case).

In the other simulation under the effect of noise (Table 2.4, Figures 2.6(a), 2.6(b), Table 2.5, and Figures 2.7(a), 2.7(b), 2.8), it was observed that as the SNR increases, performance of all the TFDs decreases. The Chirplet Transform gives excellent results if used on Doppler signals (affected by noise). Results obtained in both white Gaussian and white uniform noise cases for all signal types are very similar. Overall result of the SPWV was good, it gives highly correlated instantaneous frequency for sine (fast), linear cross, and parallel (multi component) signals at 5 and $10 \mathrm{~dB}$ level. In a good number of cases, SPWV performance is less affected by noise compared to the other TFDs.

In conclusion, performance of the TFD estimators varies depending on the input signals' characteristics such as linearity, rate of frequency change, mono or multi-component and the closeness between frequency components in the signal. For mono-component linear FM signals, almost all estimated instantaneous frequency laws are highly correlated with their 
corresponding reference. For multi-component signals, due to the effect of interference terms, the WVD and the PWVD become unreliable tools for estimating instantaneous frequency. The SPWVD and the Spectrogram have high ability to suppress interference terms. Their estimated instantaneous frequency is highly correlated with the known instantaneous frequency and less affected by white noise. The SPWVD is preferred to the Spectrogram for the image watermark application due to its better joint time-frequency resolution as a result of the advantageous smoothing kernel design. 


\begin{tabular}{|c|c|c|c|c|c|c|c|c|c|c|}
\hline Signal type & WV & PWV & SPWV & SP & d(phase) & CW & Page & Ppage & Chirplet & MP \\
\hline Linear & 0.9609 & 0.9611 & 0.9961 & 0.9848 & 1.0000 & 0.9917 & 0.8911 & 0.9747 & 0.9563 & 0.9732 \\
\hline Sine (slow) & 0.9997 & 0.9998 & 0.9997 & 0.9984 & 0.9997 & 0.9999 & 1.0000 & 0.9918 & 0.9987 & 0.9956 \\
\hline Sine (fast) & 0.9920 & 0.9922 & 0.9943 & 0.9165 & 0.9872 & 0.9913 & 0.9366 & 0.9351 & 0.1665 & 0.8276 \\
\hline FSK & 0.991 & 0.9912 & 0.9566 & 0.9232 & 0.9781 & 0.9905 & 0.9638 & 0.9733 & 0.9727 & 0.258 \\
\hline Hyperbola & 0.7778 & 0.7812 & 0.8147 & 0.7239 & 0.982 & 0.9058 & 0.6056 & 0.2852 & 0.7872 & 0.7316 \\
\hline Parabola & 0.9906 & 0.9908 & 0.9994 & 0.9959 & 0.9995 & 0.9997 & 0.9936 & 0.9577 & 0.9893 & 0.9813 \\
\hline Arbitrary & 0.9659 & 0.9662 & 0.9822 & 0.9639 & 0.9902 & 0.9815 & 0.9074 & 0.9006 & 0.9602 & 0.9778 \\
\hline FM power & 0.8917 & 0.8934 & 0.9355 & 0.8852 & 0.9922 & 0.9676 & 0.5715 & 0.5719 & 0.8959 & 0.8367 \\
\hline
\end{tabular}

Table 2.2: Correlation coefficients between the estimated and referenced instantaneous frequency resulted from various timefrequency estimators on mono-component signals 


\begin{tabular}{|c|c|c|c|c|c|c|c|c|c|c|}
\hline Signal type & WV & PWV & SPWV & SP & d(phase) & $\mathrm{CW}$ & Page & Ppage & Chirplet & MP \\
\hline Linear (crossing) & 0.1004 & 0.0994 & 0.9804 & 0.8148 & 0.6992 & 0.0688 & 0.004 & 0.1003 & 0.8994 & 0.7936 \\
\hline Linear (parallel-close) & -0.0016 & 0.0609 & 0.9942 & 0.9987 & 0.3986 & -0.0838 & 0.1128 & -0.0066 & 0.9684 & 0.9330 \\
\hline Linear (parallel-far) & 0.6924 & 0.6868 & 0.9950 & 0.9564 & 0.0050 & 0.7439 & 0.5856 & 0.1832 & 0.9450 & 0.9120 \\
\hline Sine-Hyperbola & -0.0652 & -0.0616 & 0.9780 & 0.9545 & 0.6058 & 0.0905 & 0.2256 & -0.0155 & 0.4308 & 0.7099 \\
\hline Doppler & 0.995 & 0.9952 & 0.9933 & 0.9791 & 0.9991 & 0.9993 & 0.9771 & 0.9754 & 0.9943 & 0.9863 \\
\hline Doppler-noise & 0.8769 & 0.8615 & 0.9875 & 0.9751 & 0.8755 & 0.4385 & 0.8946 & 0.7197 & 0.9738 & 0.9746 \\
\hline
\end{tabular}

Table 2.3: Correlation coefficients between the estimated and referenced instantaneous frequency resulted from various timefrequency estimators on multi-component signals 


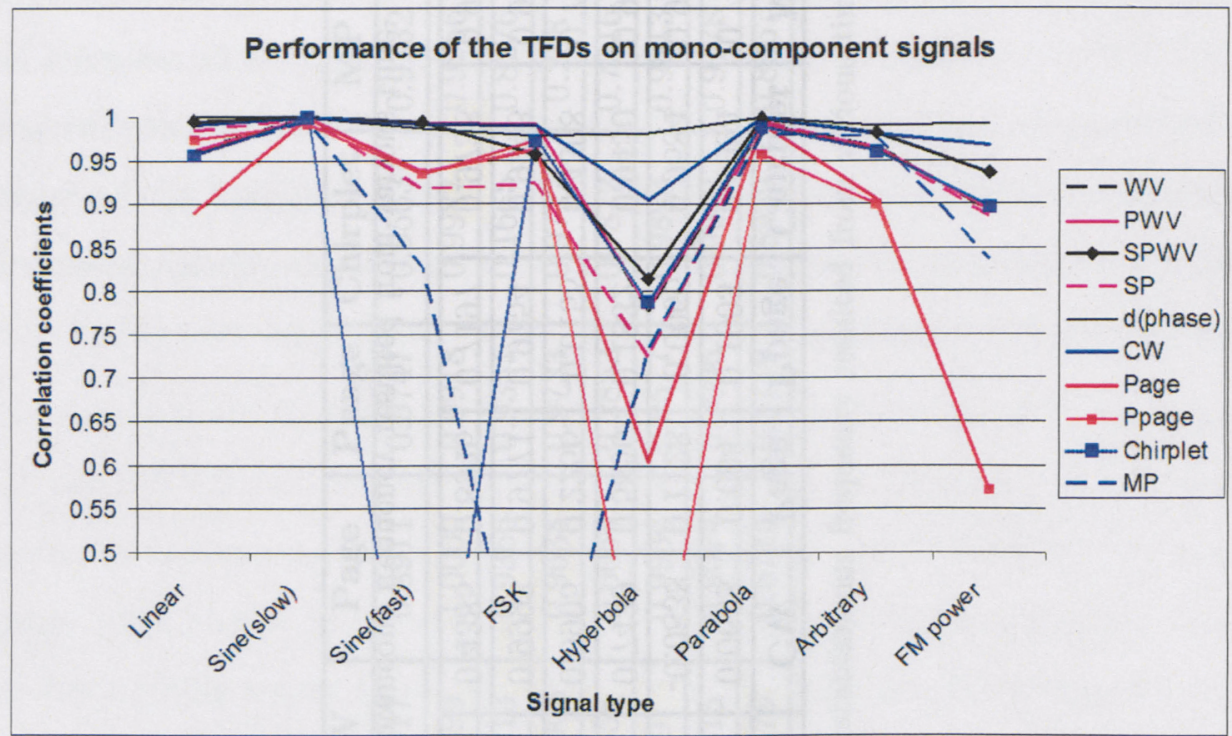

(a) Mono-component signals

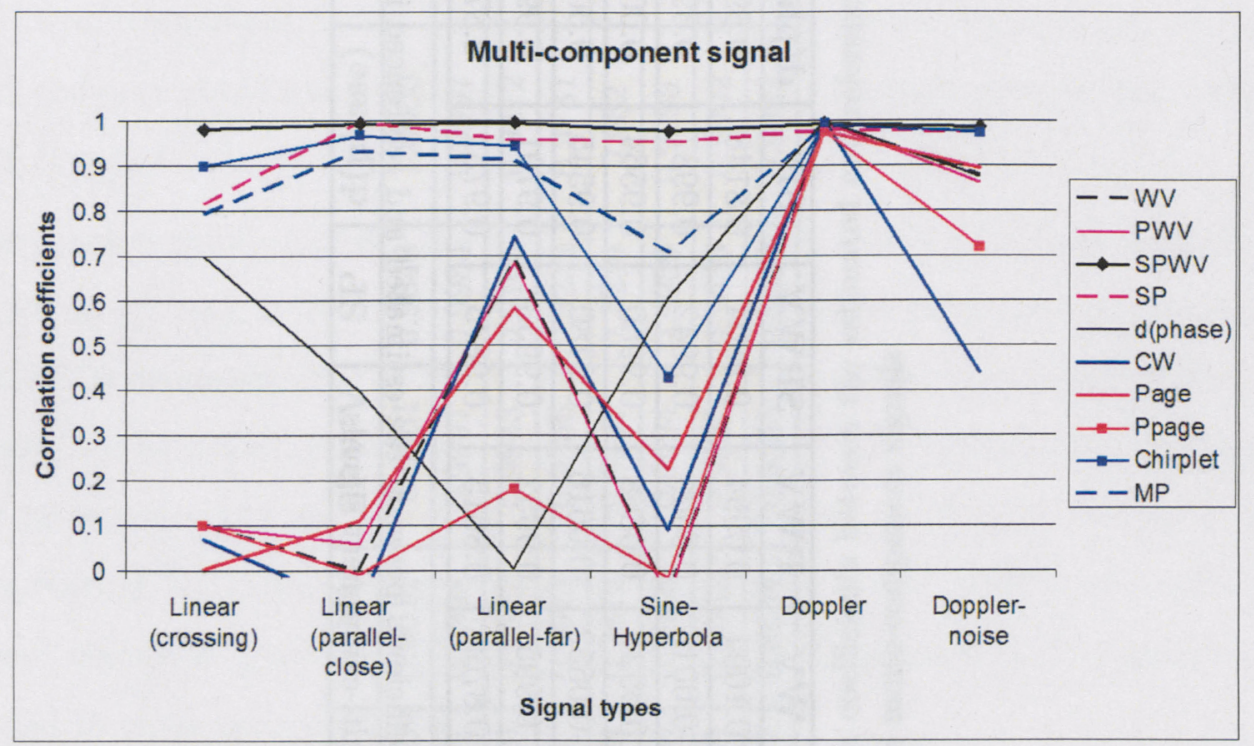

(b) Multi-component signals

Figure 2.5: Correlation coefficients between the estimated and referenced instantaneous frequency using the common TFDs 


\begin{tabular}{|c|c|c|c|c|c|c|c|c|c|c|c|}
\hline Signal type & $\mathrm{SNR}(\mathrm{dB})$ & WV & PWV & SPWV & SP & $\mathrm{d}$ (phase) & CW & Page & Ppage & Chirplet & MPD \\
\hline Linear & infinite & 0.9609 & 0.9611 & 0.9961 & 0.9848 & 1.0000 & 0.9917 & 0.8911 & 0.9747 & 0.9563 & 0.9732 \\
\hline \multirow{4}{*}{$\begin{array}{l}\text { White uniform } \\
(\mathrm{m}=0, \mathrm{var}=1)\end{array}$} & 0 & 0.2484 & 0.2533 & 0.9495 & 0.9635 & 0.2099 & 0.2674 & 0.2865 & 0.1457 & 0.9513 & 0.6984 \\
\hline & 1 & 0.3354 & 0.3295 & 0.9679 & 0.9733 & 0.2865 & 0.1301 & 0.3036 & 0.2027 & 0.9516 & 0.8299 \\
\hline & 5 & 0.7306 & 0.7363 & 0.9946 & 0.9858 & 0.5769 & 0.5989 & 0.6868 & 0.4915 & 0.9611 & 0.9338 \\
\hline & 10 & 0.8971 & 0.8972 & 0.9971 & 0.9859 & 0.7958 & 0.8992 & 0.8394 & 0.7717 & 0.9601 & 0.9707 \\
\hline \multirow{4}{*}{$\begin{array}{l}\text { White Gaussian } \\
(\mathrm{m}=0, \operatorname{var}=1)\end{array}$} & 0 & 0.1592 & 0.2024 & 0.9877 & 0.9971 & 0.2796 & 0.0244 & 0.1815 & 0.1022 & 0.9547 & 0.9150 \\
\hline & 1 & 0.2095 & 0.2207 & 0.9919 & 0.9974 & 0.3335 & 0.1626 & 0.3162 & 0.2717 & 0.9448 & 0.9351 \\
\hline & 5 & 0.6636 & 0.6656 & 0.9980 & 0.9954 & 0.5807 & 0.2515 & 0.5153 & 0.3381 & 0.9629 & 0.9636 \\
\hline & 10 & 0.8996 & 0.9004 & 0.9981 & 0.9917 & 0.7953 & 0.8866 & 0.8526 & 0.7899 & 0.9669 & 0.9755 \\
\hline Sine (fast) & infinite & 0.9977 & 0.9978 & 0.9444 & 0.1446 & 0.9880 & 0.9878 & 0.9420 & 0.9283 & 0.2879 & 0.6862 \\
\hline \multirow{4}{*}{$\begin{array}{l}\text { White uniform } \\
(\mathrm{m}=0, \mathrm{var}=1)\end{array}$} & 0 & 0.2169 & 0.2027 & 0.5658 & 0.0430 & 0.4081 & 0.3192 & 0.2726 & 0.1875 & 0.4280 & 0.4234 \\
\hline & 1 & 0.4872 & 0.4283 & 0.5371 & 0.0594 & 0.4920 & 0.3067 & 0.4351 & 0.2844 & 0.1485 & 0.3147 \\
\hline & 5 & 0.7829 & 0.7878 & 0.7759 & 0.0436 & 0.6846 & 0.6541 & 0.7136 & 0.4641 & 0.1582 & 0.634 \\
\hline & 10 & 0.9372 & 0.9374 & 0.8855 & 0.0866 & 0.9616 & 0.8798 & 0.8829 & 0.7602 & 0.7118 & 0.6272 \\
\hline \multirow{4}{*}{$\begin{array}{l}\text { White Gaussian } \\
\qquad(\mathrm{m}=0, \operatorname{var}=1)\end{array}$} & 0 & 0.2964 & 0.3336 & 0.4478 & 0.0226 & 0.4373 & 0.0706 & 0.3218 & 0.2003 & 0.2538 & 0.3154 \\
\hline & 1 & 0.1544 & 0.1600 & 0.4866 & -0.0115 & 0.4879 & 0.2657 & 0.1696 & 0.1480 & 0.0424 & 0.4185 \\
\hline & 5 & 0.7809 & 0.7772 & 0.7972 & 0.0372 & 0.7230 & 0.3049 & 0.7269 & 0.4511 & 0.1221 & 0.5957 \\
\hline & 10 & 0.9391 & 0.9392 & 0.9017 & 0.1027 & 0.9369 & 0.8665 & 0.8766 & 0.7552 & 0.4695 & 0.6125 \\
\hline
\end{tabular}

Table 2.4: Correlation coefficients between the estimated and referenced instantaneous frequency resulted from various timefrequency estimators on mono-component signals under the various levels of white Gaussian and white uniform noise 


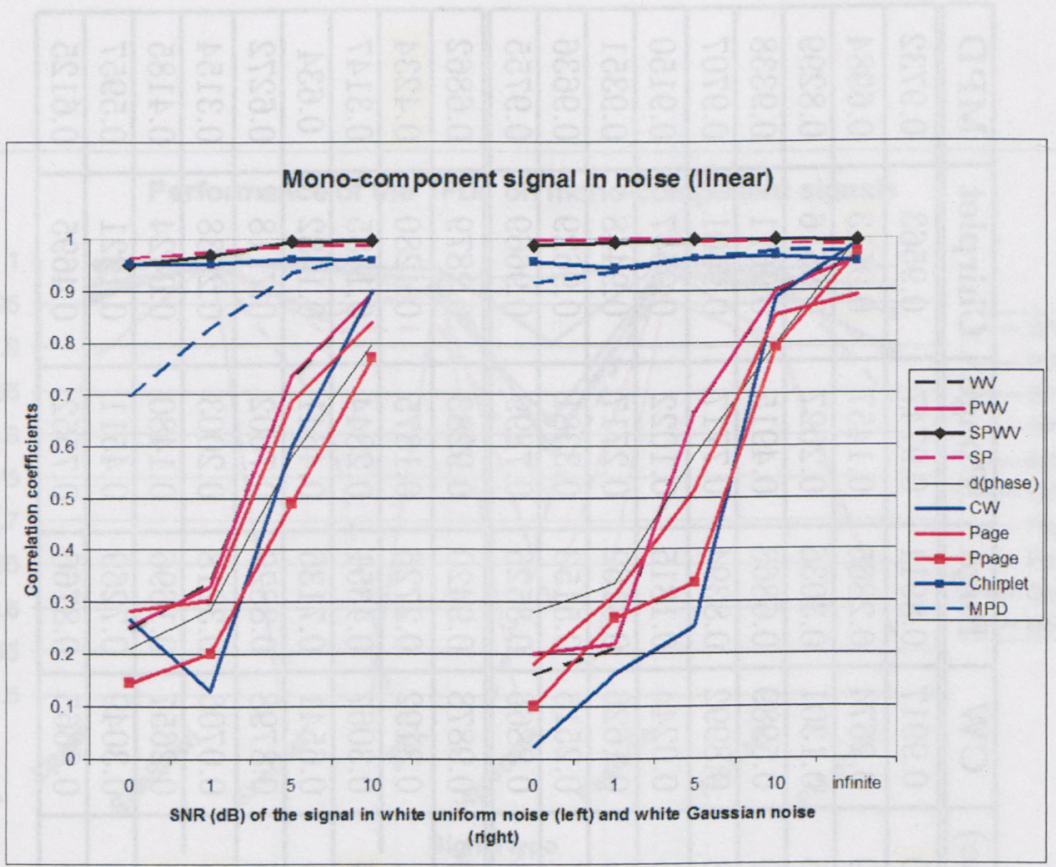

(a) Mono-component linear FM

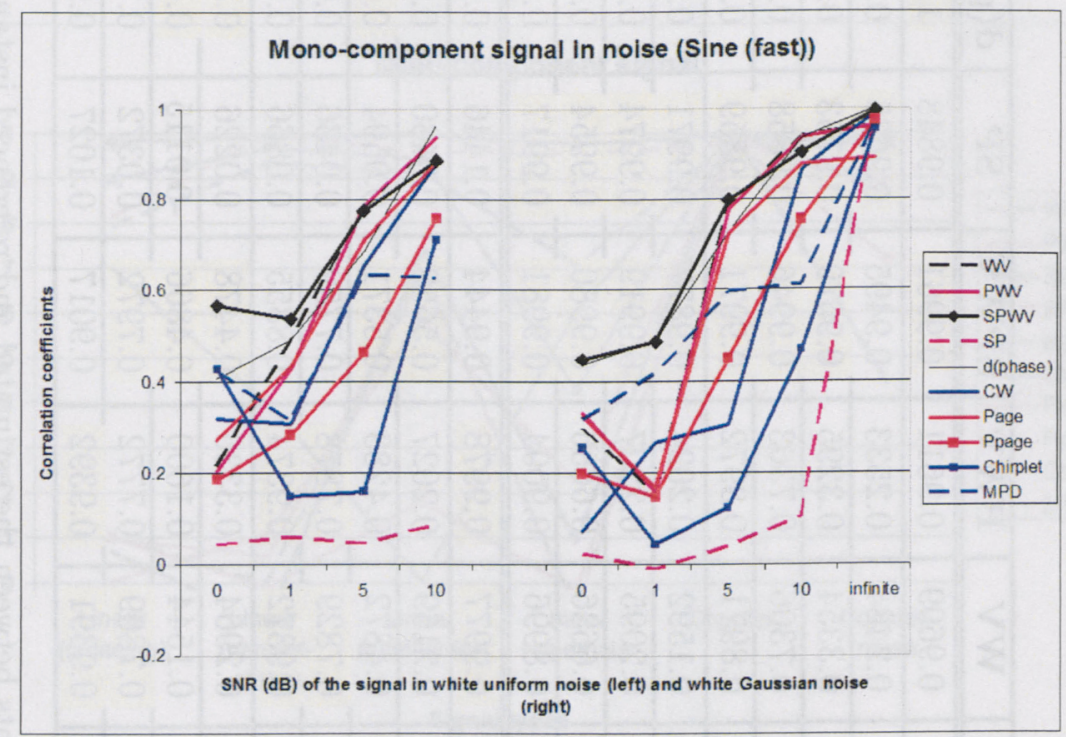

(b) Mono-component sine FM

Figure 2.6: Correlation coefficients between the estimated and referenced instantaneous frequency of different mono-component signals using the common TFDs under the effect of white uniform and white Gaussain noise 


\begin{tabular}{|c|c|c|c|c|c|c|c|c|c|c|c|}
\hline Signal type & $\mathrm{SNR}(\mathrm{dB})$ & WV & PWV & SPWV & SP & $\mathrm{d}$ (phase) & CW & Page & Ppage & Chirplet & MPD \\
\hline Crossing & infinity & -0.0522 & -0.0547 & 0.9951 & 0.9061 & 0.7006 & -0.0380 & 0.0639 & 0.0774 & 0.9308 & "0.7481 \\
\hline \multirow{4}{*}{$\begin{array}{l}\text { White uniform } \\
(\mathrm{m}=0, \operatorname{var}=1)\end{array}$} & 0 & 0.0807 & 0.0726 & 0.7745 & 0.8847 & 0.2052 & 0.0518 & 0.0905 & 0.0448 & 0.8387 & 0.3529 \\
\hline & 1 & 0.0939 & 0.0738 & 0.6881 & 0.6563 & 0.1321 & 0.0785 & 0.0402 & 0.0400 & 0.9152 & 0.2891 \\
\hline & 5 & -0.0310 & -0.0261 & 0.9028 & 0.9213 & 0.3600 & 0.1257 & 0.1223 & 0.0724 & 0.8040 & 0.4310 \\
\hline & 10 & 0.0457 & 0.0485 & 0.9727 & 0.8675 & 0.2926 & 0.0635 & 0.0574 & 0.0080 & 0.8399 & 0.5865 \\
\hline \multirow{4}{*}{$\begin{array}{l}\text { White Gaussian } \\
\qquad(\mathrm{m}=0, \operatorname{var}=1)\end{array}$} & 0 & 0.0498 & 0.0517 & 0.4756 & 0.6939 & 0.0908 & 0.0760 & 0.0511 & -0.0254 & 0.7829 & 0.4764 \\
\hline & 1 & 0.0494 & 0.0535 & 0.8183 & 0.8751 & 0.2960 & -0.0110 & 0.1627 & 0.0566 & 0.8629 & 0.1210 \\
\hline & 5 & 0.1822 & 0.1898 & 0.9334 & 0.9186 & 0.3322 & 0.0876 & 0.1968 & 0.1145 & 0.8585 & 0.4518 \\
\hline & 10 & 0.1503 & 0.1548 & 0.9325 & 0.8556 & 0.3305 & 0.1036 & 0.1702 & 0.0085 & 0.9233 & 0.8096 \\
\hline Parallel-close & infinite & -0.0018 & -0.0456 & 0.9995 & 0.9997 & 0.4273 & -0.0200 & $\mathrm{NaN}$ & $\mathrm{NaN}$ & 0.9843 & 0.9701 \\
\hline \multirow{4}{*}{$\begin{array}{l}\text { White uniform } \\
(\mathrm{m}=0, \mathrm{var}=1)\end{array}$} & 0 & 0.2563 & 0.2541 & 0.8942 & 0.9454 & 0.2997 & 0.2137 & 0.2132 & 0.1566 & 0.944 & 0.7357 \\
\hline & 1 & 0.1290 & 0.0605 & 0.9540 & 0.9772 & 0.3014 & -0.0078 & 0.2049 & 0.0830 & 0.9404 & 0.8952 \\
\hline & 5 & 0.2502 & 0.2854 & 0.9916 & 0.9961 & 0.3952 & 0.1042 & 0.3335 & 0.2239 & 0.9795 & 0.9414 \\
\hline & 10 & 0.2428 & 0.2425 & 0.9967 & 0.9981 & 0.3642 & 0.1566 & 0.3298 & 0.2291 & 0.9654 & 0.9657 \\
\hline \multirow{4}{*}{$\begin{array}{l}\text { White Gaussian } \\
(\mathrm{m}=0, \operatorname{var}=1)\end{array}$} & 0 & 0.1895 & 0.2107 & 0.9569 & 0.9848 & 0.2203 & 0.1818 & 0.1966 & 0.1947 & 0.9636 & 0.6013 \\
\hline & 1 & 0.2180 & 0.2182 & 0.9576 & 0.9728 & 0.2829 & 0.1316 & 0.2244 & 0.1436 & 0.9403 & 0.4758 \\
\hline & 5 & 0.2527 & 0.2566 & 0.9846 & 0.9924 & 0.4633 & 0.1721 & 0.3337 & 0.2076 & 0.9714 & 0.9312 \\
\hline & 10 & 0.2506 & 0.2648 & 0.9983 & 0.9987 & 0.4175 & 0.1685 & 0.3477 & 0.3211 & 0.9765 & 0.9626 \\
\hline Doppler & infinite & 0.9975 & 0.9976 & 0.9886 & 0.9718 & 0.9996 & 0.9996 & 0.9973 & 0.9877 & 0.9908 & (0.9924 \\
\hline \multirow{4}{*}{$\begin{array}{l}\text { White uniform } \\
(\mathrm{m}=0, \operatorname{var}=1)\end{array}$} & 0 & 0.0365 & 0.0195 & 0.6965 & 0.7558 & 0.2701 & 0.0446 & 0.1077 & 0.1162 & 0.9266 & 0.6972 \\
\hline & 1 & 0.2301 & 0.2279 & 0.8441 & 0.9066 & 0.3387 & 0.1943 & 0.2415 & 0.1244 & 0.9427 & 0.8327 \\
\hline & 5 & 0.5680 & 0.5746 & 0.9443 & 0.9538 & 0.6416 & 0.4537 & 0.5755 & 0.3097 & 0.9873 & 0.8445 \\
\hline & 10 & 0.8347 & 0.8313 & 0.9826 & 0.9703 & 0.9011 & 0.6630 & 0.8462 & 0.6085 & 0.9961 & 0.9688 \\
\hline \multirow{4}{*}{$\begin{array}{l}\text { White Gaussian } \\
\qquad(\mathrm{m}=0, \operatorname{var}=1)\end{array}$} & 0 & 0.2980 & 0.3000 & 0.8465 & 0.9080 & 0.3352 & 0.2736 & 0.3291 & 0.1303 & 0.9102 & 0.6401 \\
\hline & 1 & 0.2962 & 0.3068 & 0.7608 & 0.8357 & 0.3677 & 0.2181 & 0.2929 & 0.1603 & 0.9703 & 0.7247 \\
\hline & 5 & 0.5317 & 0.5170 & 0.9542 & $0.9590^{\circ}$ & 0.6842 & 0.278 & 0.5067 & 0.2932 & 0.9925 & 0.8940 \\
\hline & 10 & 0.7663 & 0.7765 & 0.9840 & 0.9743 & 0.9278 & 0.2161 & 0.7720 & 0.5767 & 0.9946 & 0.9803 \\
\hline
\end{tabular}

Table 2.5: Correlation coefficients between the estimated and referenced instantaneous frequency resulted from various timefrequency estimators on multi-component signals under the various levels of white Gaussian and white uniform noise 


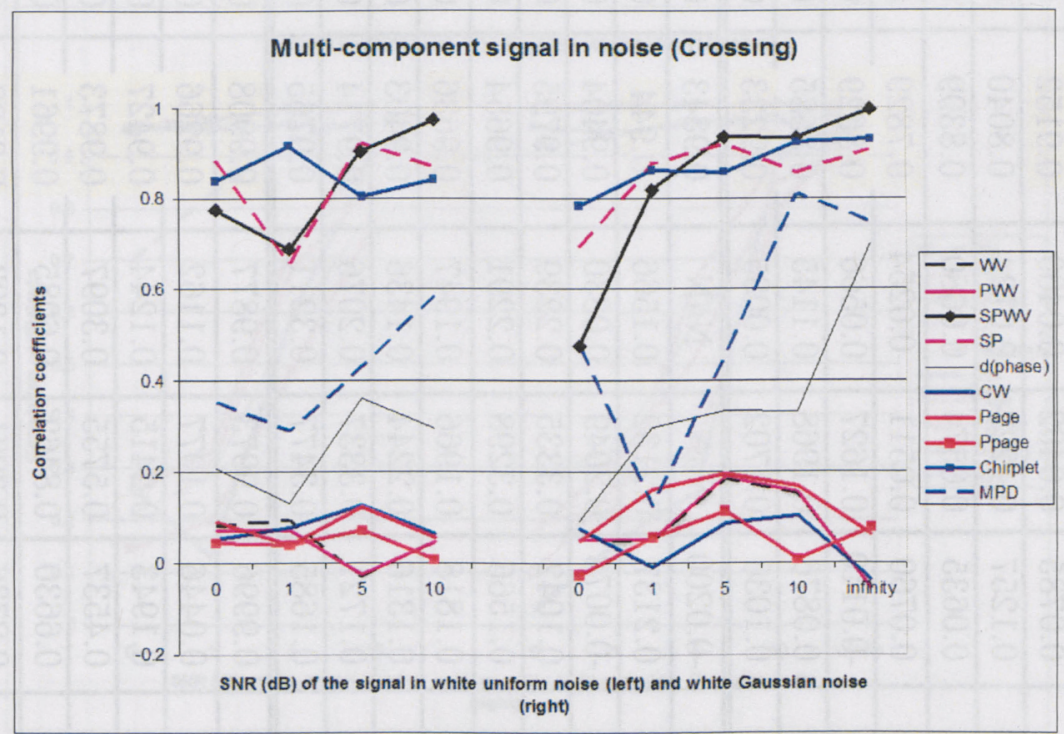

(a) Multi-component linear FM (crossing)

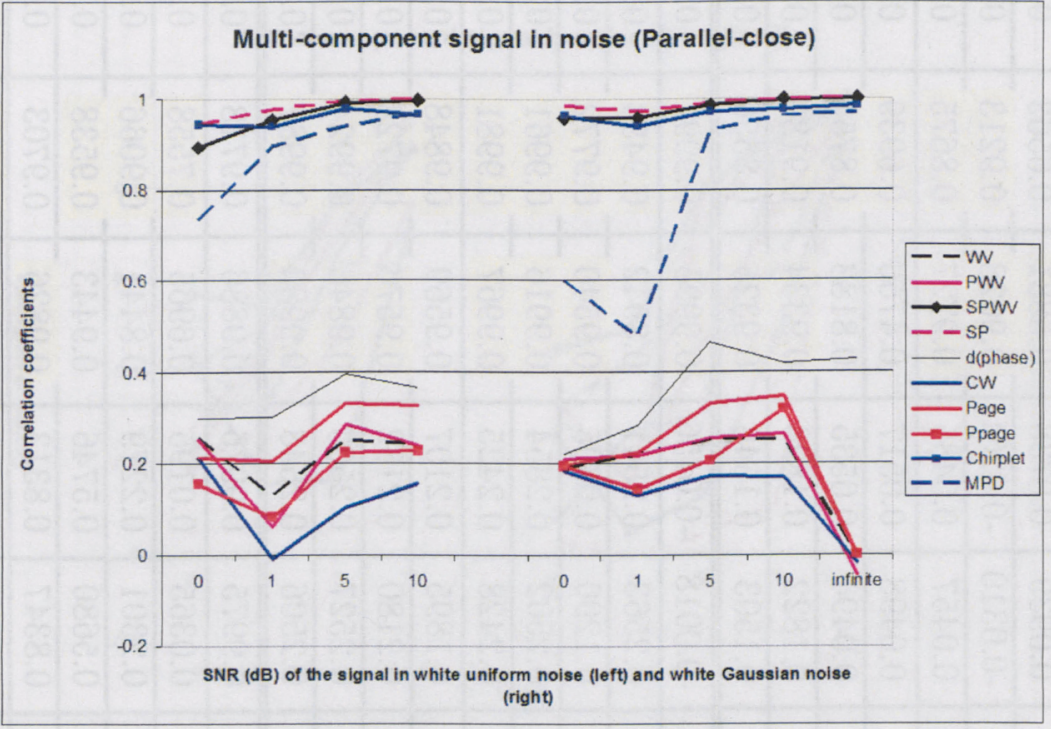

(b) Multi-component linear FM (parallel-close)

Figure 2.7: Correlation coefficients between the estimated and referenced instantaneous frequency of different multi-component signals using the common TFDs under the effect of white uniform and white Gaussain noise 


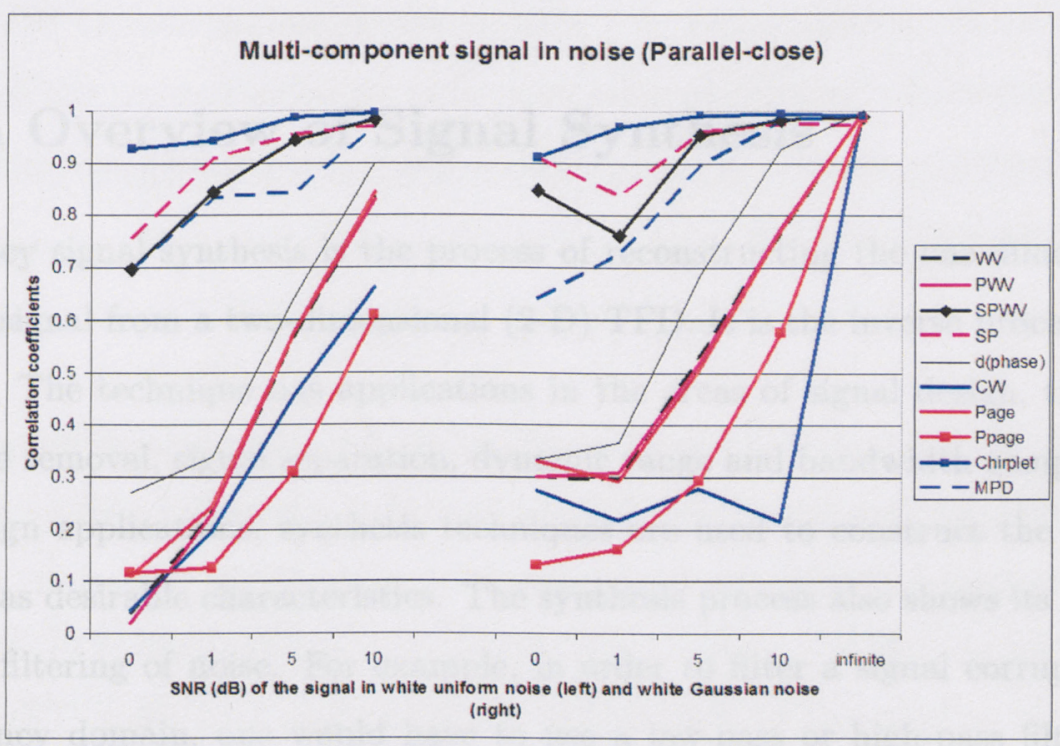

Figure 2.8: Correlation coefficients between the estimated and referenced instantaneous frequency of different multi-component signals using the common TFDs under the effect of white uniform and white Gaussain noise (Doppler signal) 



\section{Chapter 3}

\section{Signal Synthesis}

\subsection{An Overview of Signal Synthesis}

Time-frequency signal synthesis is the process of reconstructing the one-dimensional (1-D) time domain signal from a two-dimensional (2-D) TFD. It is the inverse process of calculating the TFD. The technique has applications in the areas of signal design, time-frequency filtering, noise removal, signal separation, dynamic range and bandwidth compression [3, 2]. In signal design applications, synthesis techniques are used to construct the signal from a TFD which has desirable characteristics. The synthesis process also shows its advantages in time-varying filtering of noise. For example, in order to filter a signal corrupted by noise, in the frequency domain, one would have to use a low-pass or high-pass filter. The task becomes difficult when the signal spectrum overlaps with the noise spectrum and when the noise characteristics are not available. For the same noisy signal, if the overlapping spectra of the signal and noise do not happen at the same time period, the noise can be easily removed from the signal by applying a mask or a threshold to the TFD. The clean signal can then be synthesized from the modified TFD. In signal separation applications, instead of trying to extract the signal components out of a multicomponent signal in the time domain or frequency domain, one can try to do so in the joint time-frequency domain. The idea behind this is the same as in the previous applications: the difficulties caused by overlapping of signal components in time or frequency domain can be avoided by using a TFD. The instantaneous frequency trajectory of each signal component is first separated from the TFD. 
The time domain signal of each component then can be synthesized from its modified TFD which is well localized from the others.

\subsection{The Time-Frequency Synthesis Method Based on Least Squares Approximation}

This section of the thesis will describe the significant work of Bartels and Parks to generate the signal from the WVD [3].

Assume we are given a signal $s(n)$ with its corresponding discrete WVD $W_{s}(n, f)$. In many applications, for example in noise removal or signal extraction, the distribution needs to be thresholded or masked by multiplying it with a 2-D masking function $M(n, f)$ in the time-frequency $(n, f)$ domain:

$$
Y_{s}(n, f)=W_{s}(n, f) M(n, f)
$$

The process results in distribution $Y_{s}(n, f)$ which has some desired properties. Unfortunately, $Y_{s}(t, f)$ is often not a valid WVD. Therefore, the corresponding time signal $x(n)$ can't be generated by taking the inverse operation of the WVD. Therefore, the least squares approximation method is employed to synthesize $x(n)$. Let's call the WVD of $x(n), W_{x}(n, f)$. The synthesis problem can be described as finding a signal $x(n)$ whose TFD, $W_{x}(n, f)$, best approximates a given TFD $Y_{s}(n, f)$. This requirement is equivalent to minimizing the error function $J(x)$ between $W_{x}(n, f)$ and $Y_{s}(n, f)$ :

$$
J(x)=\sum_{n} T \int\left|Y_{s}(n, f)-W_{x}(n, f)\right|^{2} d f .
$$

The solution to the minimization problem above is the even- and odd-indexed sequences $x_{e}(n) \exp \left(j \alpha_{e}\right)$ and $x_{o}(n) \exp \left(j \alpha_{o}\right)$, where parameters $\alpha_{e}$ and $\alpha_{o}$ are the phase of those sequences. The desired signal $x(n)$ is obtained by combining $x_{e}(n) \exp \left(j \alpha_{e}\right)$ and $x_{o}(n) \exp \left(j \alpha_{o}\right)$. Different values of $\alpha_{e}$ and $\alpha_{o}$ will result in different versions of the signal $x(n)$ with various signal properties because of the time delay although they have the same TFD. This problem is solved by comparing the odd- and even-indexed sequences with the original $s(n)$ to 
determine $\alpha_{e}$ and $\alpha_{o}$ so that it will minimize the phase difference. So reading in the original sequence $s(n)$ and comparing it with the previous estimated $x_{e}(n)$ and $x_{o}(n)$ to determine the right values for the phase coefficients $\alpha_{e}$ and $\alpha_{o}$ :

$$
\begin{aligned}
& \alpha_{e}=\tan ^{-1}\left[\frac{\operatorname{Re}\left[\sum_{n} s(2 n) x_{e}^{*}(n)\right]}{\operatorname{Im}\left[\sum_{n} s(2 n) x_{e}^{*}(n)\right]}\right], \\
& \alpha_{o}=\tan ^{-1}\left[\frac{\operatorname{Re}\left[\sum_{n} s(2 n-1) x_{o}^{*}(n)\right]}{\operatorname{Im}\left[\sum_{n} s(2 n-1) x_{o}^{*}(n)\right]}\right] .
\end{aligned}
$$

The drawback of this method is the matching process of the two odd- and even-sequences as mentioned above. This drawback can be avoided by using the synthesis technique based on EDTWD as in $[16,17,18,19,20,21]$.

\subsection{The Problem of Phase Reconstruction and The Need For Initial Information From The Signals}

From Equation (2.4) in Section 2.2, a TFD is calculated by taking the Fourier Transform of the energy of the signal and applying a smoothing operation on it. Multiplication of the signal and its conjugate pair destroys the phase information of the signal. And this is why the time domain signal $x(n)$ can be recovered from its corresponding TFD up to some constant phase factor $\alpha$ only [6]:

$$
\hat{x}(n)=x(n) e^{j \alpha}
$$

Since different values of $\alpha$ will result in different versions of $x(n)$ with the same TFD, recovery of the original signal with the same original phase factor is possible only if some initial information on the signal phase is available. That is why the synthesis algorithms based on the TFD always need to determine the exact phase term by comparing the estimated output $\hat{x}(n)$ with the original sequence $s(n)$. The DPPT algorithm discussed in the next section is another approach which can synthesize the signal $x(n)$ from $s(n)$, where $s(n)$ 
could be a corrupted version of $x(n)$ or a combination of $x(n)$ with other signal components. The difference between the two synthesis techniques lies in the fact that the DPPT uses a polynomial to model the signals' phase instead of the TFD of the signals. A more detailed discussion of the method is discussed in the following section and later on, another section will describe a technique which can combine the usage of both methods to synthesize the signal component for chirp message detection in the watermark application.

\subsection{The Synthesis Method Based on The DPPT}

The DPPT has been extensively studied in recent years [32, 33, 34]. It is a parametric signal analysis approach for estimating the phase parameters of polynomial phase signals. Normally, the phase parameters of a signal are determined by applying least squares approximation to fit a polynomial to the phase curve. This process poses some difficulty especially when the phase is not available. The DPPT, on the other hand, is applied directly onto the signal and it works quite well even in the presence of noise. The phase $\phi(n)$ of many man-made signals such as those used in radar, sonar, communications can be modeled as a polynomial. The discrete version of a polynomial phase signal can be expressed as:

$$
\begin{aligned}
x(n) & =b_{0} \exp \{j \phi(n)\} \\
& =b_{0} \exp \left\{j \sum_{m=0}^{M} a_{m}(n \Delta)^{m}\right\},
\end{aligned}
$$

where $M$ is the polynomial order (For e.g., $M=2$ for linear chirp, and $M=3$ for parabolic chirp signals), $N$ is the total signal length and $\Delta$ is the sampling interval.

The principle of the DPPT is as follows: when the DPPT of order $M$ is applied to a mono-component signal with polynomial phase of order $M$, it produces a spectral peak. The position of this spectral peak at frequency $\omega_{0}$ provides a way to estimate the coefficient $\hat{a}_{M}$. After $\hat{a}_{M}$ is estimated, the order of the polynomial is reduced from $M$ to $M-1$. The next coefficient $\hat{a}_{M-1}$ is estimated the same way by taking the DPPT of order $M-1$ of the polynomial phase signal of order $M-1$ above. The procedure is repeated until all the coefficients of the polynomial phase are found. 
The DPPT of order $M$ of a continuous phase signal $x(n)$ is defined as the Fourier Transform of the higher order $\mathcal{D} \mathcal{P}_{M}[x(n), \tau]$ operator $[32,33,34]$ :

$$
\begin{aligned}
D P P T_{M}[x(n), \omega, \tau] & \equiv \mathcal{F}\left\{\mathcal{D} \mathcal{P}_{M}[x(n), \tau]\right\} \\
& =\sum_{(M-1)^{\tau}}^{N-1} \mathcal{D} \mathcal{P}_{M}[x(n), \tau] \exp ^{-j \omega n \Delta},
\end{aligned}
$$

where $\tau$ is a positive number and:

$$
\begin{aligned}
\mathcal{D P} & {[x(n), \tau] } \\
\mathcal{D P}_{2}[x(n), \tau] & :=x(n) \\
\mathcal{D} \mathcal{P}_{M}[x(n), \tau] & :=\mathcal{D P}_{2}\left[\mathcal{D} \mathcal{P}_{M-1}[x(n), \tau], \tau\right]
\end{aligned}
$$

$\mathcal{D P} \mathcal{P}_{2}$ operator has phase differencing effect on the signal:

$$
\mathcal{D P} \mathcal{P}_{2}=b_{0} \exp \{j[\phi(n)-\phi(n-\tau)]\}
$$

After the phase is differentiated, the order of the polynomial will be reduced by one. From Equation (3.8), if $\mathcal{D} \mathcal{P}_{M}$ is calculated on the phase polynomial $\phi(n)$ of order $M$, the order of the polynomial will be reduced $M-1$ times. This results in a new polynomial of order one whose coefficient is proportional to the coefficient $a_{M}$ of the original polynomial $\phi(n)$. Therefore, taking Fourier Transform of $\mathcal{D} \mathcal{P}_{M}$ will result in a spectral peak at $\omega_{0}$, and this $\omega_{0}$ value will help to determine the coefficient $a_{M}$.

Figure 3.1 shows the result of the DPPT operation of different orders on a linear chirp having its phase modeled as a second order polynomial. Figure (3.1(a)) is the SPWVD of the chirp signal. Figures 3.1(b), 3.1(c), and 3.1(d) show the first, second and third order DPPT of the signal in Figure 3.1(a) respectively. The spectral peak only appears in the case of second order DPPT corresponding to the second order of the signal phase.

The coefficients $a_{M}$ is estimated based on the following formula:

$$
\hat{a}_{M}=\frac{1}{M !\left(\tau_{M} \Delta\right)^{M-1}} \operatorname{argmax}_{\omega}\left\{\left|D P P T_{M}[x(n), \omega, \tau]\right|\right\},
$$




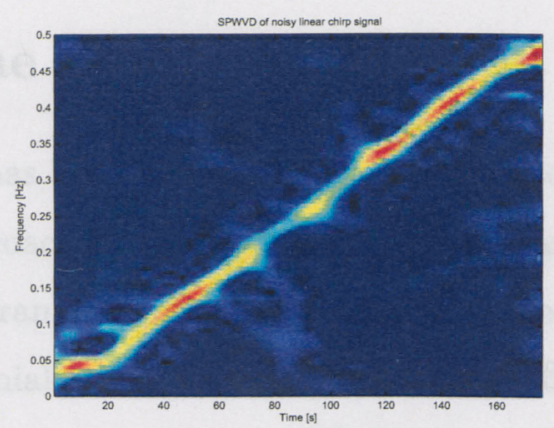

(a) TFD of chirp signal

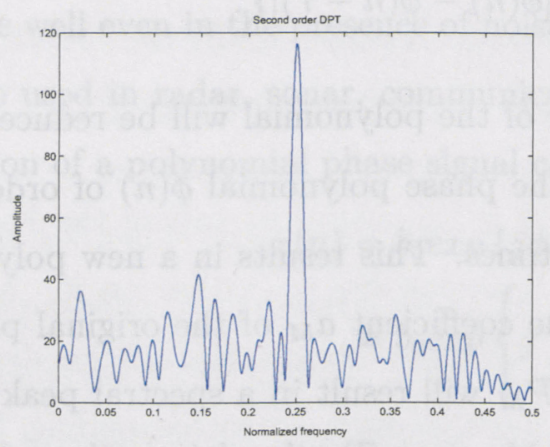

(c) Second order DPPT

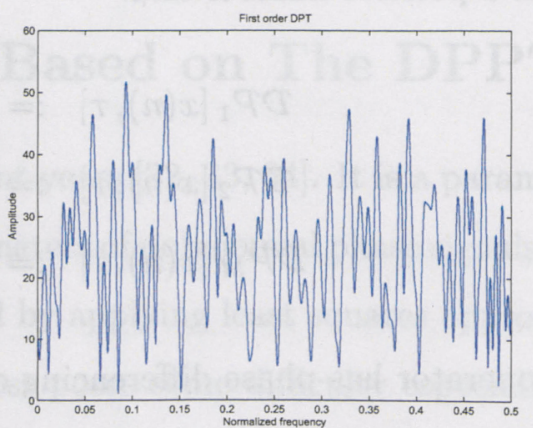

(b) First order DPPT

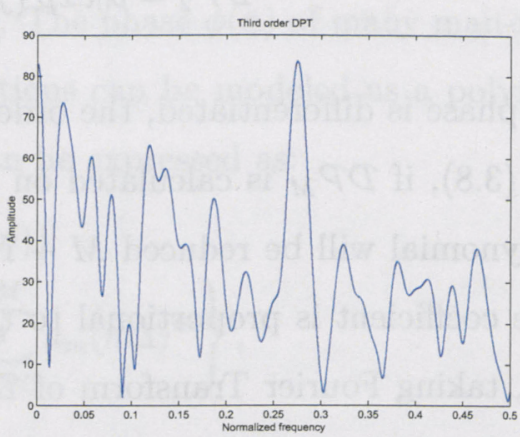

(d) Third order DPPT

Figure 3.1: DPPT of linear chirp signal with BER of $5 \%$ 
where $D P P T_{M}[x(n), \omega, \tau]$ is calculated as in Equation (3.8). The formulas for the DPPT of order from one to three are shown below:

$$
\begin{aligned}
D_{P P T_{1}}[x(n), \omega, \tau] & =\mathcal{F}\{x(n)\} \\
D_{P P T_{2}}[x(n), \omega, \tau] & =\mathcal{F}\left\{x(n) x^{*}(n-\tau)\right\} \\
D_{P P T_{3}}[x(n), \omega, \tau] & =\mathcal{F}\left\{x(n)\left[x^{*}(n-\tau)\right]^{2} x(n-2 \tau)\right\}
\end{aligned}
$$

After $a_{M}$ is estimated, the order of the signal phase will be reduced by multiplying the signal $x(n)$ with $\exp \left\{-j \hat{a}_{M}(n \Delta)^{M}\right\}$ :

$$
x(n)^{(M-1)}=x(n) \exp \left\{-j \hat{a}_{M}(n \Delta)^{M}\right\}
$$

This reduction of order is called phase unwrapping. To determine $a_{M-1}$, apply the DPPT of order $M-1$ on the signal $x(n)^{(M-1)}$ from the previous equation. The whole process is repeated until all coefficients $a_{M}(M>1)$ are calculated. Coefficient $a_{0}$ and $b_{0}$ are determined by the following formulas:

$$
\begin{aligned}
& \hat{a_{0}}=\text { phase }\left\{\sum_{n=0}^{N-1} x(n) \exp \left\{-j \sum_{m=1}^{M} a_{m}(n \Delta)^{m}\right\}\right\}, \\
& \hat{b}_{0}=\frac{1}{N} \sum_{n=0}^{N-1} x(n) \exp \left\{-j \sum_{m=1}^{M} a_{m}(n \Delta)^{m}\right\} .
\end{aligned}
$$

The estimated coefficients $\hat{a}_{M}$ and $b_{0}$ are used to synthesize the polynomial phase signal $\hat{x}(n)$ :

$$
\hat{x}(n)=\hat{b}_{0} \exp \left\{j \sum_{m=0}^{M} \hat{a}_{m}(n \Delta)^{m}\right\} .
$$

\subsection{Synthesis of Chirp Signals}

This section describes in more detail how to synthesize the chirp signal using the above method. The precision of the estimated coefficients depends on the chosen value of $\tau[33,34]$. The mean square error between the estimated and referenced highest order coefficient $a_{M}$ was analyzed for a wide range of $\tau / N$ values. The least error is achieved when $\tau=N / M$, 


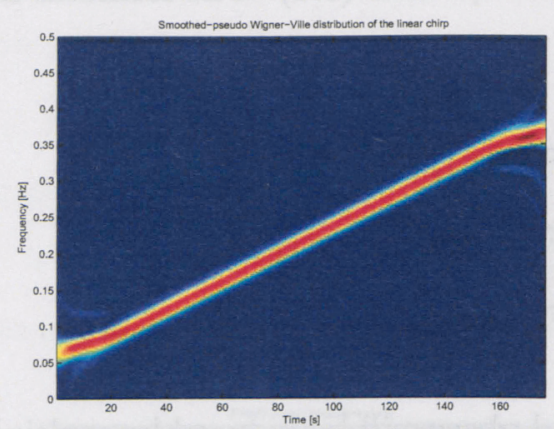

(a) TFD of linear chirp signal

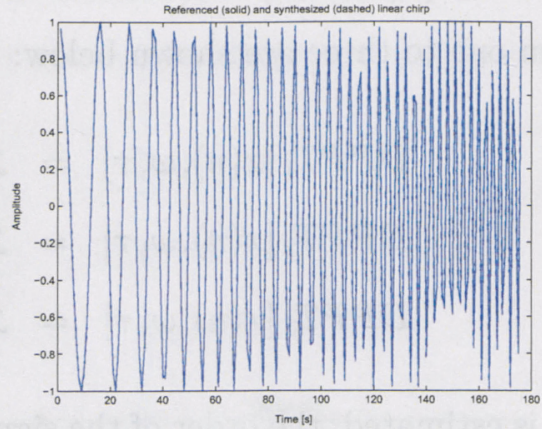

(b) Referenced and synthesized linear chirp

Figure 3.2: Referenced (solid) and synthesized (dashed) versions of the linear chirp

where $N$ is the length of the signal of interest and $M$ is the order of the polynomial phase of the chirp signal. A linear frequency modulated chirp has its phase modeled as a second order polynomial. Similarly, a parabolic frequency modulated chirp (non-linear chirp) has its phase modeled as a third order polynomial. The current watermark application uses chirp signal with constant amplitude (normalized), therefore $b_{0}=1$.

Figures 3.2 and 3.3 show the TFD of the linear chirp and parabola chirp as well as the referenced and estimated signals in the time domain. In these examples, the estimated version of the chirp signals are highly correlated with their referenced counterparts. The accuracy of the estimation process can be seen through the examination of the coefficients' value in Table 3.1.

It is important to keep in mind that in order for the DPPT synthesis algorithm to work, the location $\omega_{0}$ of the spectral peak when taking the Fourier Transform of the $\mathcal{D} \mathcal{P}$ operator has to be smaller than half of the sampling frequency $\omega_{s}$ :

$$
\left|\omega_{0}\right|=M !(\tau \Delta)^{M-1}\left|a_{M}\right| \leq \frac{\omega_{s}}{2} .
$$

This condition is translated into the following requirement on the range of coefficient $a_{M}$ :

$$
\left|a_{M}\right| \leq \frac{\pi}{M ! \tau^{M-1} \Delta^{M}}
$$




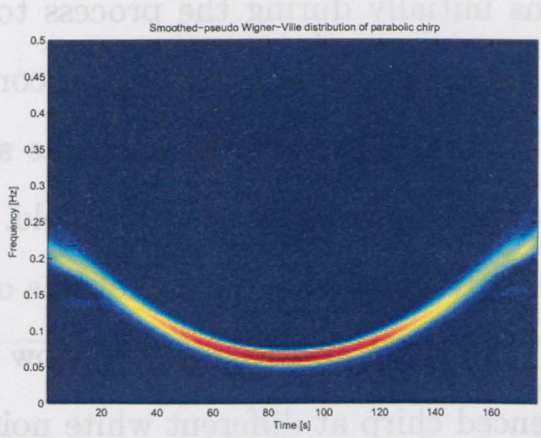

(a) TFD of parabolic chirp signal

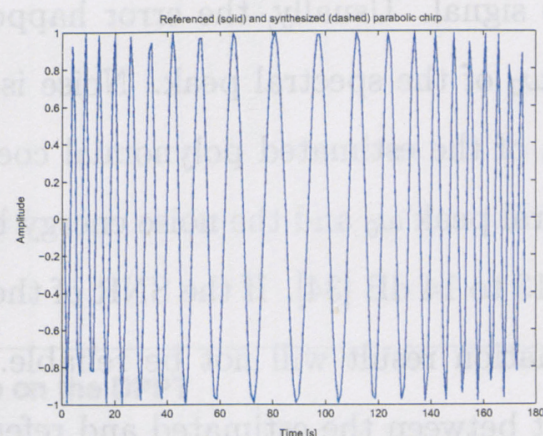

(b) Referenced and synthesized parabolic chirp

Figure 3.3: Referenced (solid) and synthesized (dashed) versions of the parabolic chirp

\begin{tabular}{|c|c|c|c|}
\hline Signal & Coefficient & Referenced & Synthesized \\
\hline \multirow{3}{*}{ Linear chirp } & $a_{2}$ & 0.0060 & 0.0060 \\
\cline { 2 - 4 } & $a_{1}$ & 0.3000 & 0.3007 \\
\cline { 2 - 4 } & $a_{0}$ & 0.0100 & -0.0539 \\
\hline \multirow{4}{*}{ Parabolic chirp } & $a_{3}$ & $5.000 e^{-005}$ & $5.0031 e^{-005}$ \\
\cline { 2 - 4 } & $a_{2}$ & -0.0130 & -0.0130 \\
\cline { 2 - 4 } & $a_{1}$ & 1.5000 & 1.5002 \\
\cline { 2 - 4 } & $a_{0}$ & 0.1000 & 0.1157 \\
\hline
\end{tabular}

Table 3.1: Polynomial coefficients of the referenced and the synthesized chirp signals 
when $M=1$, we have $\left|a_{1}\right| \leq \frac{\omega_{s}}{2}$ which is the Nyquist criterion.

The accuracy of the estimation process discussed above can be affected by several other factors such as the level of noise present, the type of noise, the slope and the length of the chirp signal. Usually, the error happens initially during the process to determine the location $\omega_{0}$ of the spectral peak. Noise is the number one factor which contributes to the deviation of the estimated polynomial coefficients. The ratio between the signal energy at the spectral peak $\omega_{0}$ and the noise energy has to be greater than a threshold. This threshold is about 13 to $14 \mathrm{~dB}$ [34]. If the SNR of the analysis signal is outside of this operating range, the estimation result will not be reliable. Table 3.2 and Figure 3.4 show the correlation coefficient between the estimated and referenced chirp at different white noise levels.

Increasing the order of the polynomial also increases the estimation error. For example, if we consider a third order polynomial, any error which occurs in coefficient $a_{3}$ will make it impossible to remove this coefficient completely from the polynomial during phase unwrapping step. Therefore, the estimation of the next coefficient $a_{2}$ will suffer and have error as well. Similarly, the new error in $a_{2}$ will affect the precision of $a_{1}$ and $a_{0}$. When the length of the chirp signal is increased, any small error in the estimated coefficients will be propagated when applying Equation (3.17) to generate the synthesized chirp. This propagation of error will also significantly reduce the correlation coefficient between the referenced and estimated signal. 


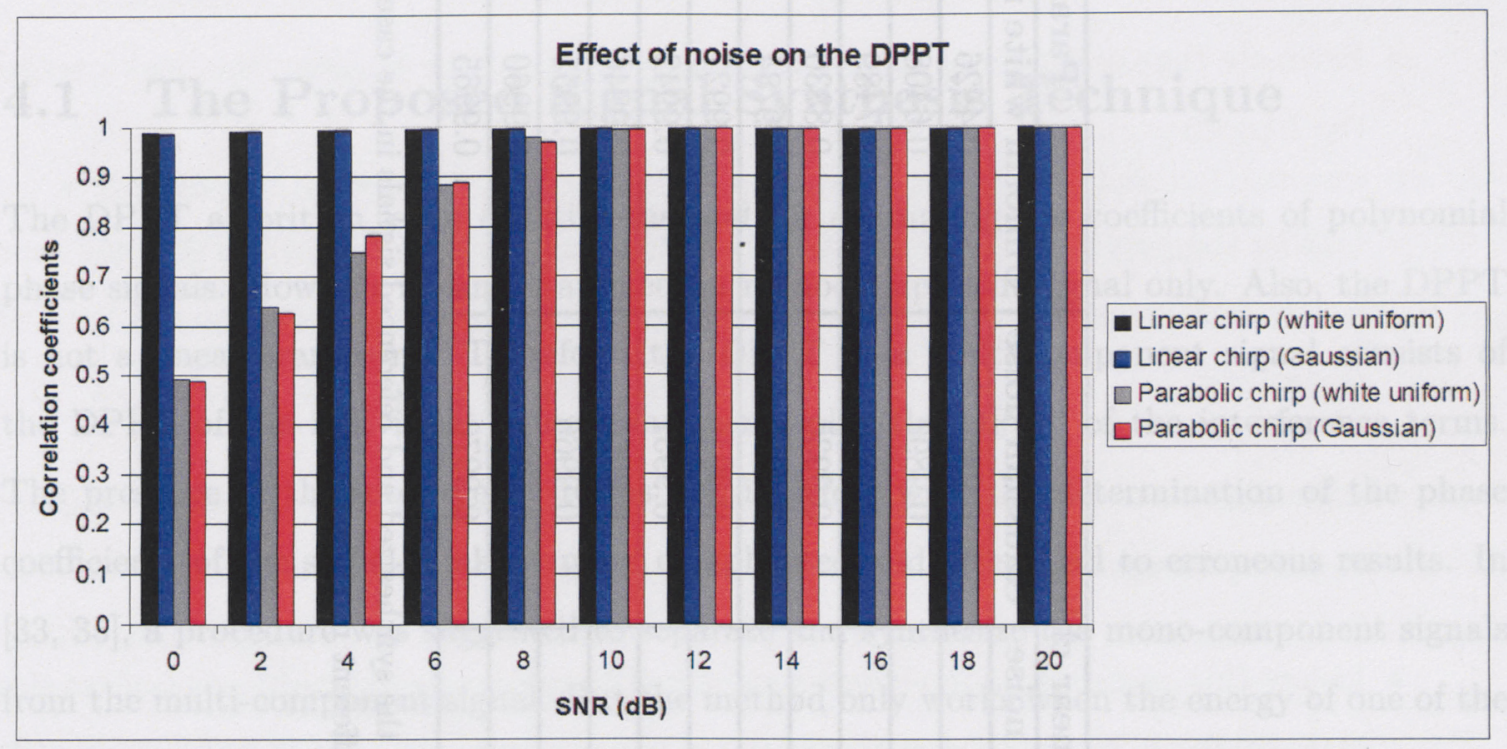

Figure 3.4: Correlation coefficients between the synthesized and referenced chirps under the effect of white uniform and white Gaussian noise at various SNRs 


\begin{tabular}{|c|c|c|c|c|}
\hline \multirow{2}{*}{ SNR $(\mathbf{d B})$} & \multicolumn{2}{|c|}{ Linear chirp } & \multicolumn{2}{c|}{ Parabolic chirp } \\
\cline { 2 - 5 } & Uniform white noise & Gaussian noise & Uniform white noise & Gaussian noise \\
\hline 0 & 0.9890 & 0.9867 & 0.4926 & 0.4874 \\
\hline 2 & 0.9903 & 0.9897 & 0.6408 & 0.6273 \\
\hline 4 & 0.9921 & 0.9919 & 0.7489 & 0.7830 \\
\hline 6 & 0.9932 & 0.9936 & 0.8830 & 0.8881 \\
\hline 8 & 0.9942 & 0.9939 & 0.9784 & 0.9669 \\
\hline 10 & 0.9947 & 0.9948 & 0.9927 & 0.9926 \\
\hline 12 & 0.9955 & 0.9957 & 0.9942 & 0.9935 \\
\hline 14 & 0.9960 & 0.9960 & 0.9946 & 0.995 \\
\hline 16 & 0.9964 & 0.9966 & 0.9955 & 0.9949 \\
\hline 18 & 0.9966 & 0.9965 & 0.9960 & 0.9960 \\
\hline 20 & 0.9971 & 0.9970 & 0.9965 & 0.9963 \\
\hline
\end{tabular}

Table 3.2: Correlation coefficient between the synthesized and referenced signals in the cases of linear chirp and parabolic chirp from Figures (3.2(a)) and (3.3(a)) under different noise levels and noise types 


\section{Chapter 4}

\section{Chirp Synthesis and Detectors}

\subsection{The Proposed Signal Synthesis Technique}

The DPPT algorithm is an effective method for estimating the coefficients of polynomial phase signals. However it can be applied on mono-component signal only. Also, the DPPT is not a linear transform. Therefore, the DPPT of a multi-component signal consists of the DPPT of the individual mono-components plus the DPPT of the interference terms. The presence of the interference terms will interfere with the determination of the phase coefficients of the signal, make it more complicated and often lead to erroneous results. In $[33,35]$, a procedure was suggested to separate and synthesize the mono-component signals from the multi-component signal. But the method only works when the energy of one of the signal components is much stronger than the energy of the other components. In this case, the spectral peak of the corresponding coefficients $a_{M}$ of the stronger component always has higher energy than those of the weaker components. This makes it possible for the strongest signal component to be synthesized first and then subtracted from the multi-component signal. The next strongest signal component can be determined by applying the DPPT algorithm on the residual from the subtraction. This procedure is repeated until all the signal components are estimated. When the energy of the individual component does not differ much from each other, the method has problems in determining which spectral peak belongs to which component signal, and therefore the method fails.

In order to synthesize the mono-components of a multi-component signal, a raw esti- 
mation process of each component is needed to separate the individual components from the multi-component. The DPPT algorithm is then applied on the just estimated monocomponent signal to obtain the precise values of the coefficients.

In order to obtain the initial estimation of the individual component, the method in [32] combines the DPPT algorithm with the minimum-cross entropy TFD (MCE-TFD). The algorithm is described as followed (Figure 4.1):

- Take the MCE-TFD of the initial multi-component signal.

- Estimate the trajectory of the instantaneous frequency by tracking the peak energy of the frequency ridge in the TFD.

- Obtain the polynomial representing the instantaneous frequency $\left(f_{m}(t)\right)$ of the monocomponent using least squares fit technique on the estimated instantaneous frequency trajectory.

- After $f_{m}(t)$ is determined, calculate the phase polynomial $\phi_{m}(t)$ of the mono-component signal by integrating $f_{m}(t)$ as derived from Equation (2.1).

- Isolate this particular mono-component signal from the original multi-component signal by multiplying the multi-component signal with $e^{-j \phi_{m}(t)}$. As a result, the corresponding mono-component is translated into the low frequency band.

- Low-pass filter the result to separate the individual component from the multicomponent.

- Translate the output of the low-pass filter back to the high frequency region by multiplying the filter output with $e^{j \phi_{m}(t)}$. The result of this multiplication is the first time-domain estimation of the mono-component signal. This estimation of the particular mono-component signal is then subtracted from the original multi-component signal. 


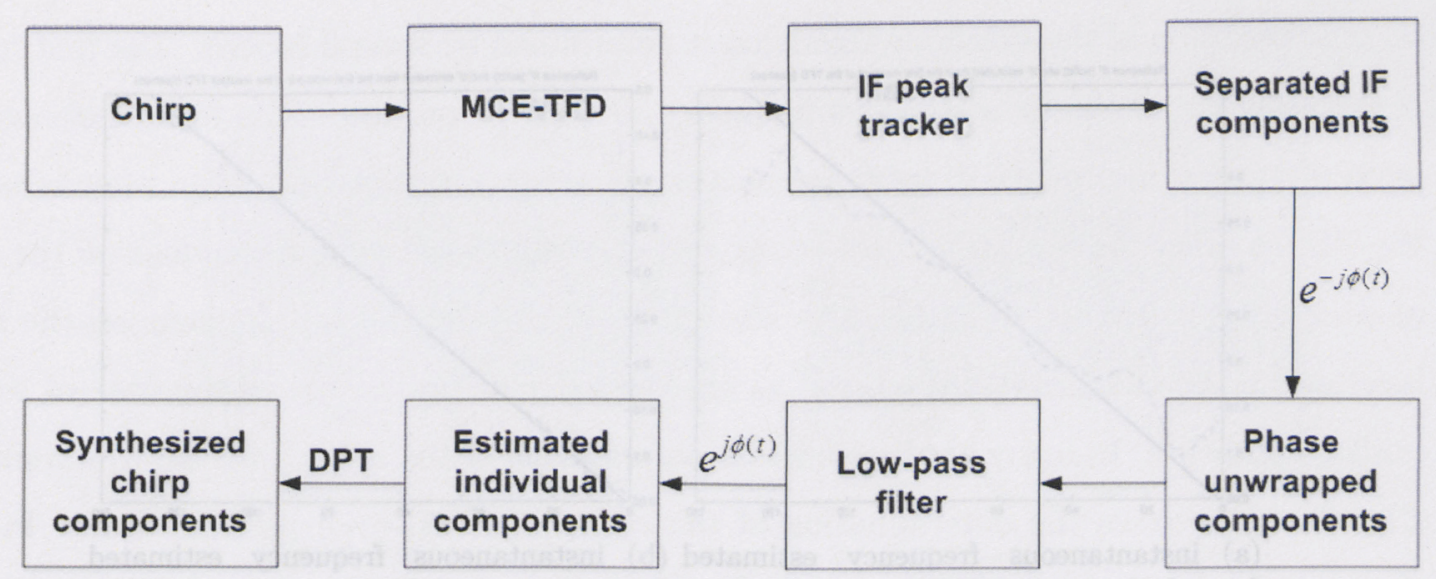

Figure 4.1: The MCE-TFD-based chirp detection method

- Repeat the same procedure to estimate all the remaining individual components. After all the individual components of the original signal are estimated, apply DPPT algorithm on each of the components to have a more precise estimation of all the polynomial coefficients for the components.

The method proposed in this thesis synthesizes the polynomial phase chirp signal using a combination of the TFD's property as well as the DPPT. This approach and the one in [32] both utilize Equation (2.1) to estimate the signal phase from the instantaneous frequency. But the method in this thesis uses the SPWVD as a tool for time-frequency representation of the signal. In addition, instead of using peak tracking algorithm to estimate the instantaneous frequency, the approach proposed in this thesis utilizes a useful property of the TFD to generate instantaneous frequency. The instantaneous frequency can be simply obtained by taking the first moment of the TFD as in Equation (2.9). The advantage of estimating instantaneous frequency using first moment of the TFD over peak tracking of the TFD is that it allows masking of the TFD, or time-frequency filtering, to either reduce the effect of noise or to estimate the instantaneous frequency laws of the mono component [36]. In other words, estimating instantaneous frequency by the first moment of the TFD extends the application of the synthesis algorithm to low SNR signals. Figure 4.2 shows the instantaneous frequency resulted from the first moment of the TFD of a $5 \mathrm{~dB}$ SNR signal in white 


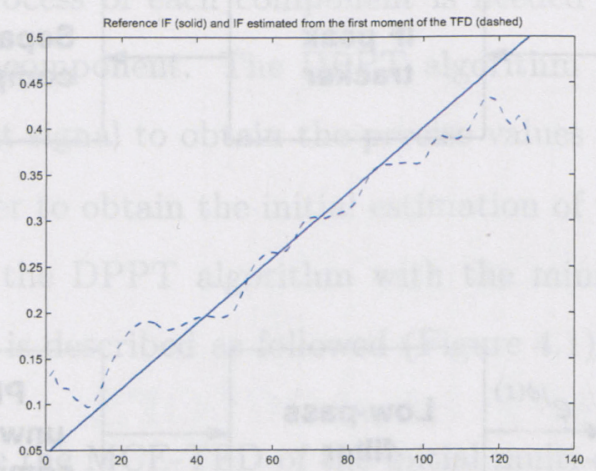

(a) instantaneous frequency estimated from the unmasked TFD

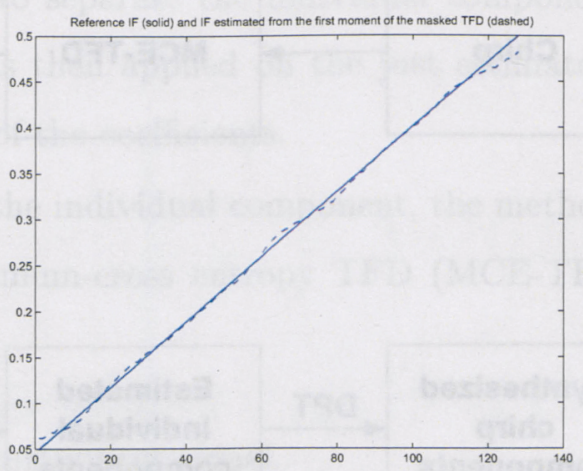

(b) instantaneous frequency estimated from the masked TFD

Figure 4.2: Effect of time-frequency filtering on instantaneous frequency estimation. 4.2(a): no time-frequency filtering: lower correlation (0.9861) between estimated (dashed) and referenced (solid) instantaneous frequency, and 4.2(b): with time-frequency filtering: high correlation (0.9993) between estimated (dashed) and referenced (solid) instantaneous frequency. The SNR of the original signal is $5 \mathrm{~dB}$

uniform noise. The instantaneous frequency estimation performed on the time-frequency filtered TFD is highly correlated with the referenced instantaneous frequency (correlation coefficient $=0.9993)$, while the instantaneous frequency estimated from the unmasked TFD has lower correlation coefficient $(0.9861)$ with the referenced instantaneous frequency. The instantaneous frequency of the individual signal can be determined by either masking of the TFD or using the algorithm in [37]. The advantage of using the SPWVD is also obvious. The distribution was well studied in Chapter 2 under the effect of different noise levels and noise types for many types of mono- and multi-component signals. In addition, the SPWVD has high joint time-frequency resolution with excellent interference term suppression ability. More importantly, the simulation in Chapter 2 also indicated the instantaneous frequency estimated by taking its first moment is highly correlated with the referenced instantaneous frequency laws. This properly makes the SPWVD an ideal candidate for using in the initial estimation of the mono-component signal.

Figure 4.3 describes the synthesis process of a chirp signal: 


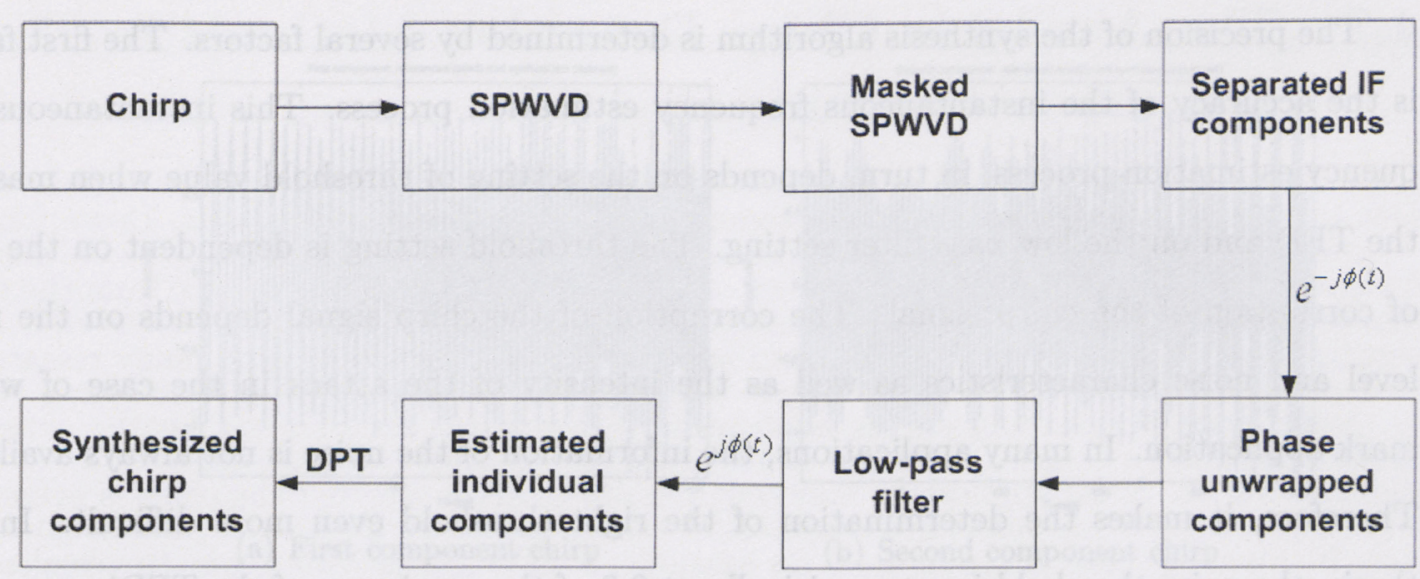

Figure 4.3: The proposed chirp detection method bases on the DPPT and SPWVD

- First, SPWVD of the original signal is taken.

- Then the TFD is masked by applying a threshold on it. The value of the threshold depends on the characteristics of the noise present such as noise's type, amplitude and distribution. The masking process removes the noise in the time-frequency plane, hence increases the signal SNR.

- The blind component-separation procedure in [37] can be applied on the time-frequency filtered TFD to have an estimate of the TFD of the mono-component signals. The components can also be separated by carefully applying the appropriate masks on the TFD of the multi-component signal.

- Estimate the instantaneous frequency of the individual component by taking the first moment of the TFD of the individual signal estimated from the above step.

- Translate the individual component to low frequency band, low pass filter and translate the outcome back to the high frequency band to obtain the estimation of the individual component.

- Apply DPPT algorithm on the separated components to have a final accurate estimated value of the phase coefficients. 
The precision of the synthesis algorithm is determined by several factors. The first factor is the accuracy of the instantaneous frequency estimation process. This instantaneous frequency estimation process, in turn, depends on the setting of threshold value when masking the TFD and on the low pass filter setting. The threshold setting is dependent on the level of corruption of the chirp signal. The corruption of the chirp signal depends on the noise level and noise characteristics as well as the intensity of the attack in the case of watermark application. In many applications, the information of the noise is not always available. Therefore, it makes the determination of the right threshold even more difficult. In this thesis, the noise threshold is set empirically at 0.8 of the maximum of the TFD's energy. A more adaptive way of setting the right threshold is beyond the scope of this thesis and it is one of the objectives for future work. The filter types, IIR and FIR, as well as their order are also of important consideration. As a rule of thumb, increasing the filter order would result in a better cutoff but at the expense of longer delay and non-linearity on the filter's response. At the same order, an IIR filter will give a sharper cutoff region but an IIR filter also has higher tendency of becoming unstable. It is also important to use sufficient number of points when taking the Fourier Transform of the ambiguity function. The simulations show that using insufficient number of points when taking the Fourier Transform results in less accurate position of the spectral peaks and this leads to the error in the estimation of the polynomial phase coefficients. The other factors which affect the synthesis algorithm are the value of $\tau$ and the length of the signal. As mentioned in Section 3.5, for second and third order polynomial chirp signals, setting $\tau=N / M$ will result with the least error in the synthesis outcome. After all the coefficients are estimated, the time domain chirp signal is synthesized using Equation (3.17), it is obvious that any error in the coefficients $a_{M}$ will propagate throughout the length of the signal, as $t$ increases, the synthesized version of the chirp will become less correlated with the original chirp signal.

Figure 4.4 shows the synthesized mono-component chirp signals from a multi-component signal. This example on a multi-component signal consists of two linear FM signals. Based on the available estimation of the instantaneous frequency of the first chirp, the algorithm 


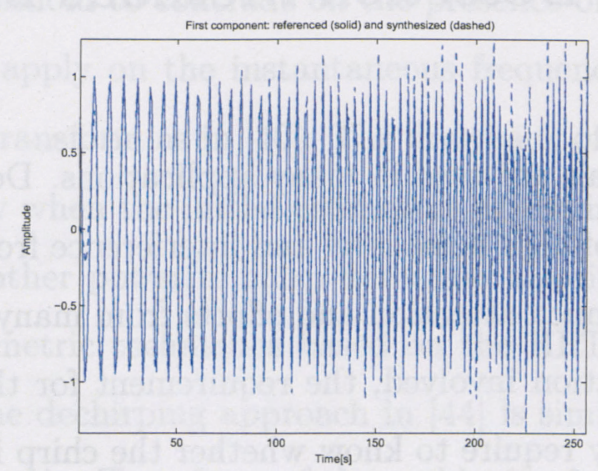

(a) First component chirp

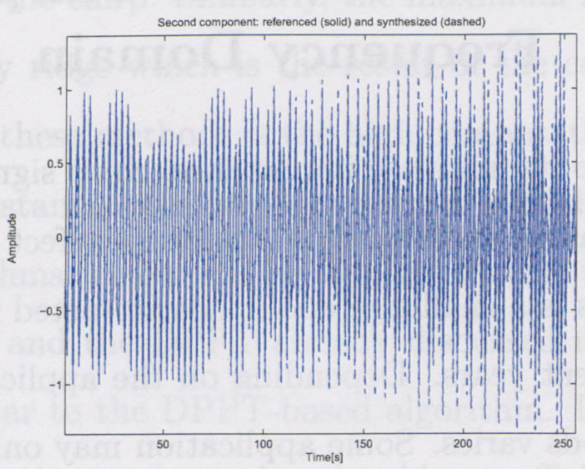

(b) Second component chirp

Figure 4.4: Synthesized (dashed) and referenced (solid) version of the individual component chirps of the multi-component signal

\begin{tabular}{|c|c|c|c|}
\hline Component & Coefficient & Referenced & Synthesized \\
\hline \multirow{3}{*}{ First chirp } & $a_{2}$ & 0.0030 & 0.0030 \\
\cline { 2 - 4 } & $a_{1}$ & 0.7540 & 0.7547 \\
\cline { 2 - 4 } & $a_{0}$ & 0 & -0.5377 \\
\hline \multirow{3}{*}{ Second chirp } & $a_{2}$ & 0.0020 & 0.0020 \\
\cline { 2 - 4 } & $a_{1}$ & 1.8850 & 1.8837 \\
\cline { 2 - 4 } & $a_{0}$ & 0 & -0.8881 \\
\hline
\end{tabular}

Table 4.1: Polynomial coefficients of the referenced and the synthesized chirp signal component from the multi-component signal

successfully separated the individual chirps from the original signal. Table 4.1 gives the comparison among the chirp coefficients of the synthesized and referenced signals. The estimation of coefficients $a_{2}$ and $a_{1}$ is very accurate, a slight error occurs when it comes to estimation of $a_{0}$, but the contribution of this error to the synthesis result is minimal. The reason is that this error is not amplified by multiplying the coefficient $a_{0}$ with the time parameter during the reconstruction of the signal from the phase coefficients. 


\subsection{The Available Chirp Detection Methods in Time- Frequency Domain}

Due to its immense importance, chirp signals are used in many applications. Detection and estimation of chirp signal under the effect of high noise level and interference from the other signals is a challenge and it has attracted much attention and efforts from many researchers in recent years. Depending on the application involved, the requirement for the detection methods varies. Some application may only require to know whether the chirp is present or not. If this is the case, a simple tool, for example, a TFD can provide a way to visualize the presence of the chirp. But in many other applications, where visualization becomes difficult due to effect of strong noise, or where there is the need to characterize the signal parameters such as the initial and end frequency, start phase and amplitude, more sophisticated detection methods are obviously needed.

One of the traditional approaches for chirp detection technique is the optimal technique [38]. The signal in question is compared with the referenced chirp by taking the squared inner product between them. The processing domain for the inner product calculation could be the time domain, frequency domain, or joint time-frequency domain [38, 39]. Other alternatives for the inner product calculation are the cross-correlation and cross-ambiguity function [40]. The coefficients resulting from these calculation are compared to a threshold to make the decision if the chirp signal is present. These methods are known to have a high rate of false alarm or non-detection possibility when the signal of analysis is heavily corrupted or affected by strong noise. The other disadvantage involves the possible high computational complexity when processing is done in the time-frequency domain.

Other methods for detecting the chirp signal are the adaptive filter approach [41], the filter bank approach [42], the evolutionary algorithm [43], and the maximum likelihood estimation. The maximum likelihood is an optimal technique which integrates along the instantaneous frequency ridge in the TFD. If initial information on the position of the instantaneous frequency is not available, the integration will be taken along all possible lines in the time-frequency domain. Results of the integration is then compared with a prede- 
fined threshold to conclude on the presence of the chirp. Similarly, the maximum likelihood can also apply on the instantaneous frequency ridge which is the result of the continuous wavelet transform as in [40]. The drawback of these methods is the high computational cost especially when the initial estimation of the instantaneous frequency is not available.

The other powerful chirp detection algorithms which will be detailed in this thesis are the parametric techniques based on the HRT and the DPPT already discussed in Section (3.4). The dechirping approach in [44] is similar to the DPPT-based algorithm. Instead of taking Fourier Transform of the ambiguity function to determine the chirp coefficients, [44] dechirps the signal of interest with $e^{-j(1 / 2) m t^{2}}$ for all different values of $\mathrm{m}$, takes the Fourier Transform for each outcome $f_{m}(t)$ and gets the power spectra $\left|F_{m}(\omega)\right|^{2}$. The right coefficient $m$ is the one whose corresponding power spectra is maximal.

The next Chapter of this thesis describes the process of using linear FM signals as a watermark message in an image watermark application, and also shows the process of how to detect them. 


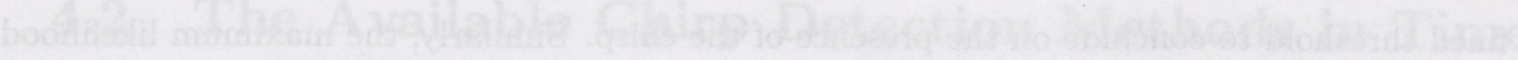

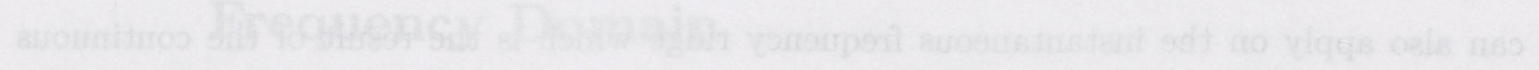

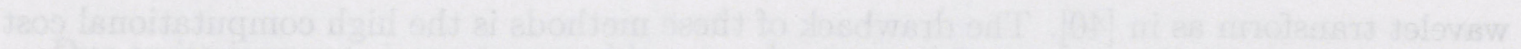

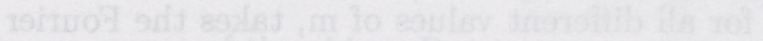

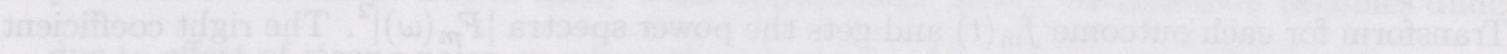

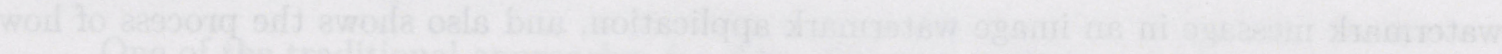

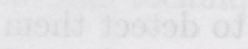

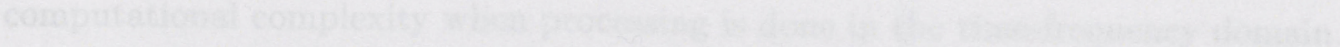

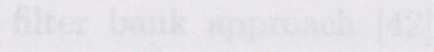

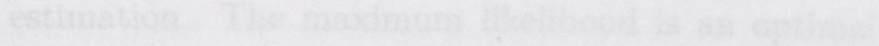

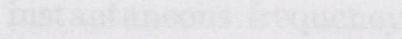

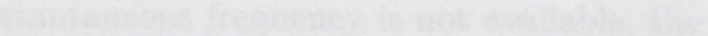




\section{Chapter 5}

\section{Applications in Multimedia Security}

\subsection{Linear Frequency Modulated Chirp as a Water- mark Message and Detection Methods}

Time-Frequency signal synthesis has been used in many areas such as time-varying filtering, signal separation, time-frequency noise reduction, mitigation of a jamming signal in radar applications, error correction, and biomedicine such as newborn EEG seizure simulation [22]. The synthesis algorithms are also used to estimate the instantaneous frequency of a signal and to design a signal whose TFD satisfies some desirable properties. One of the most common application of signal synthesis is the synthesis and detection of a chirp signal which has applications in many areas. In this thesis, time-frequency signal synthesis is applied to detect the chirp signals embedded as a watermark message in multimedia watermarking applications.

Digital watermarking is the process involves integrating a special message into digital contents such as audio, video and image for copyright protection purposes. The embedded data is then extracted from the multimedia content as a proof of ownership. Various digital watermarking methods have been researched by many authors in the past years [45]. The watermark techniques differ depending on their applications and characteristics such as invisibility, robustness, security and media category. In addition, watermark methods can also be classified by the type of watermark message used as well as the processing domain [45]. The watermark message used can be either noise type: i.e., pseudo noise sequence, Gaussian 
random sequence or classification in the form of binary images, stamps, and logos. The processing domain, where the insertion and extraction of watermark takes place, is usually spatial domain or frequency domain. The techniques based on frequency domain such as the Discrete Cosine Transform (DCT), wavelet and Fourier Transform have become popular recently. However few works have been done to exploit the unique properties and advantages of watermarking in the joint time-frequency domain.

In [46], watermark insertion and extraction are both done in time-frequency domain. In the embedding process, the watermark message $w(t, f)$, in time-frequency domain, is added to the cells of WVD $X(t, f)$ of the signal $x(t)$. The locations of the cells are carefully selected so that the message will be invisible when inverting the watermarked Wigner distribution back to the spatial domain. In the detection process, the WVD of the original message is subtracted from that of the watermarked message to retrieve the watermark.

The fragile watermark approach proposed in [47] does not require the whole original signal to recover the watermark. A quadratic chirp is modulated with a pseudo random (PN) sequence before being added to the diagonal pixels of the image in the spatial domain. Only the original value of the diagonal pixels are enough for recovering the watermark bits. After removing the PN effect, the watermark pattern can be analyzed using a TFD.

In $[25,26]$, a novel watermarking method was introduced using a linear-chirp-based technique and applied it on image and audio signals. The watermark embedding process is described as follows:

- The chirp signal $x(t)$ (or $\underline{\mathbf{m}}$ ) is quantized to have a value of -1 and 1 as in $\mathbf{m}^{q}$.

- Each bit $m_{k}^{q}$ of $\mathbf{m}^{q}$ is spread with a cyclic shifted version $\mathbf{p}_{\mathbf{k}}$ of a binary PN sequence called watermark key. The results are summed together to generate the wideband noise vector $\mathbf{w}$ :

$$
\mathbf{w}=\sum_{k=0}^{N} m_{k}^{q} \mathbf{p}_{\mathbf{k}},
$$

where $N$ is the number of watermark message bits in $\mathbf{m}^{\mathrm{q}}$.

- The wide band noise $\mathbf{w}$ is then carefully shaped and added to the audio or the DCT 
block of the image so that it will cause imperceptible change in signal quality. In the audio watermarking application in [26], to make the watermark message imperceptible, the amplitude level of the wideband noise $\mathbf{w}$ is scaled down to be about 0.3 of the dynamic range of the signal. In the image watermarking application in [25], the length of $\mathbf{w}$ to be embedded depends on the perceptual entropy of the image.

- To embed the watermark into the image, the model based on the just noticeable difference (JND) paradigm was utilized. The JND model based on the DCT was used to find the perceptual entropy of the image and to determine the perceptually significant regions to embed the watermark. In this method, the image is decomposed into $8 X 8$ blocks. Taking the DCT on the block $b$ results in the matrix $X_{u, v, b}$ of the DCT coefficients. The watermark embedding scheme is based on the model proposed in [48]. The watermark encoder for the DCT scheme is described as

$$
X_{u, v, b}^{*}=\left\{\begin{array}{lr}
X_{u, v, b}+t_{u, v, b}^{C} w_{u, v, b}, & \text { if } X_{u, v, b}>t_{u, v, b}^{C} \\
X_{u, v, b}, & \text { otherwise }
\end{array}\right.
$$

where $X_{u, v, b}$ refers to the DCT coefficients, $X_{u, v, b}^{*}$ refers to the watermarked DCT coefficients, $w_{u, v, b}$ is obtained from the wideband noise vector $\mathbf{w}$, and the threshold $t_{u, v, b}^{C}$ is the computed JND determined for various viewing conditions such as minimum viewing distance, luminance sensitivity and contrast masking.

Figure 5.1 describes how the chirp message is embedded as a watermark in an audio or image file. Figure 5.2 shows the original image, the chirp used as watermark message, and the watermarked image based on our approach. The watermark is well hidden in the image that it is imperceptible and causes no difference in the histogram. The presence of the chirp message is undetectable in the spatial and time-frequency domain thanks to the perceptual shaping processing.

The detection scheme for the DCT-based watermarking can be expressed as

$$
\begin{aligned}
\hat{w}_{u, v, b} & =\frac{X_{u, v, b}-\hat{X}_{u, v, b}^{*}}{t_{u, v, b}^{C}} \\
\hat{\mathbf{w}} & =\left\{\begin{array}{lr}
\hat{w}_{u, v, b}, & \text { if } X_{u, v, b}>t_{u, v, b}^{C} \\
0 & \text { otherwise }
\end{array}\right.
\end{aligned}
$$


where $\hat{X}_{u, v, b}^{*}$ are the coefficients of the received watermarked image, and $\hat{\mathbf{w}}$ is the received wideband noise vector. The watermark key, $\mathbf{p}_{\mathbf{k}}$ is used to despread $\hat{\mathbf{w}}$, and integrate the resulting sequence to generate a test statistic $\left\langle\hat{\mathbf{w}}, \mathbf{p}_{k}\right\rangle$. The sign of the expected value of the statistic depends only on the embedded watermark bit $m_{k}^{q}$. Hence the watermark bits can be estimated using the decision rule:

$$
\hat{m}_{k}^{q}= \begin{cases}+1, & \text { if }\left\langle\hat{\mathbf{w}}, \mathbf{p}_{k}\right\rangle>0 \\ -1, & \text { if }\left\langle\hat{\mathbf{w}}, \mathbf{p}_{k}\right\rangle<0\end{cases}
$$

The bit estimation process is repeated for all the transmitted bits. Due to intentional and non-intentional attacks such as lossy compression, shifting, and down-sampling, the received chirp message $\hat{\mathbf{m}}^{q}$ will be different from the original message $\mathbf{m}^{q}$ by a Bit Error Rate (BER). Line detection algorithms such as the ones based on the HRT and the proposed signal synthesis in this thesis are then applied on the TFD of $\hat{\mathbf{m}}^{q}$ to determine whether it is the chirp message under examination based on parameter such as chirp rate, and initial frequency.

\subsection{HRT-Based Detection}

The HRT was proposed in [30] by combining the Hough Transform and the Radon transform. The resulting transform can be applied to gray-level images and can be implemented to identify any feature that satisfies a parametric constraint. HRT can be applied to detect the instantaneous frequency patterns from a wavelet domain [49] or from a TFD. It is the only chirp detector in the time-frequency domain for the chirp embedded as watermark in multimedia security applications $[25,26]$. The quantization of the Hough space and the ability to utilize an array of accumulators make the transform become flexible and computationally attractive. HRT is an effective method for detecting, and error correcting linear chirps and it can be applied on small image of the time-frequency plane.

The Hough Transform $[50,51,52,30]$ is a feature detection method used to detect patterns like a line or a curve in an image by transforming the data space (called the image space) into a parameter space (called the Hough space). The mapping from the data space to Hough 


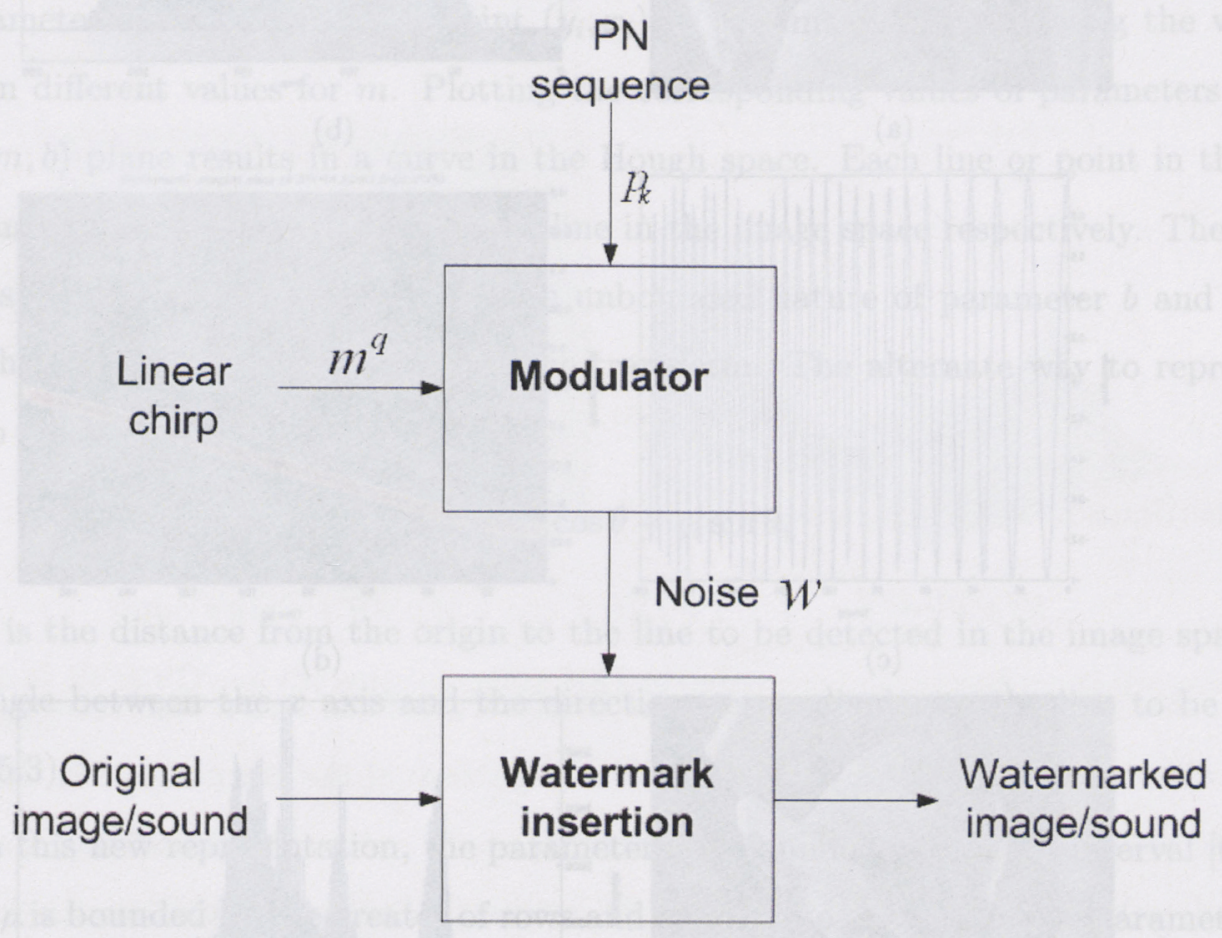

Figure 5.1: The process of embedding chirp message in audio/image file 


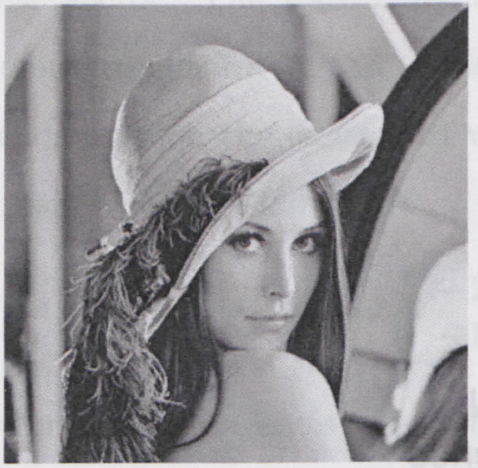

(a)

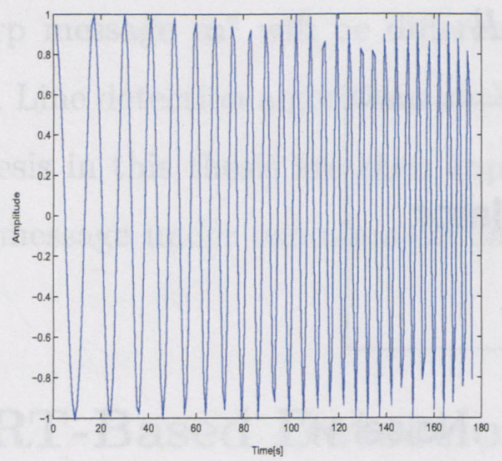

(c)

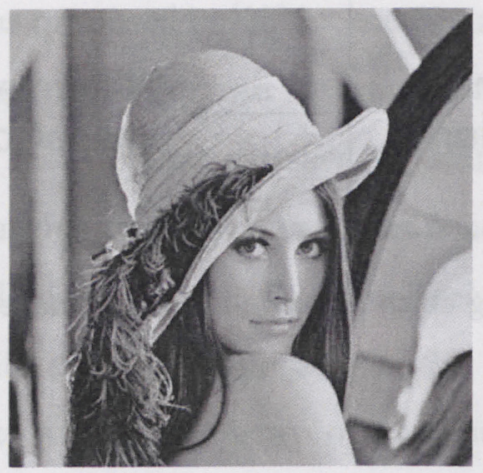

(e)

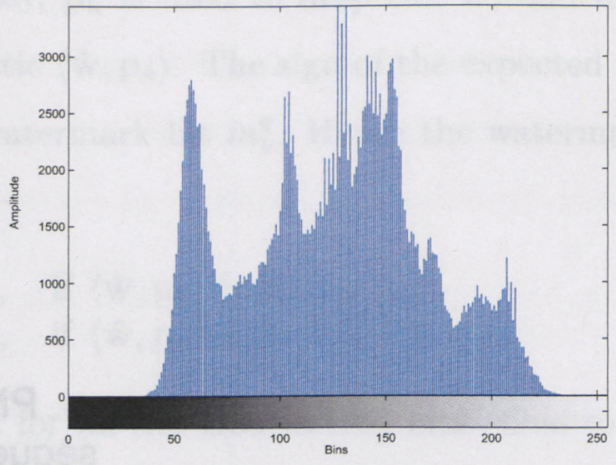

(b)

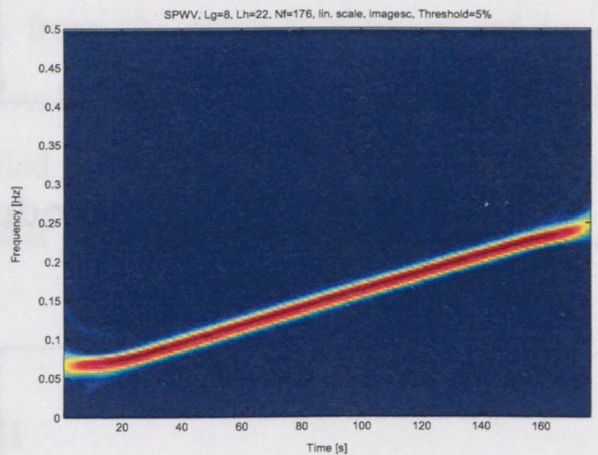

(d)

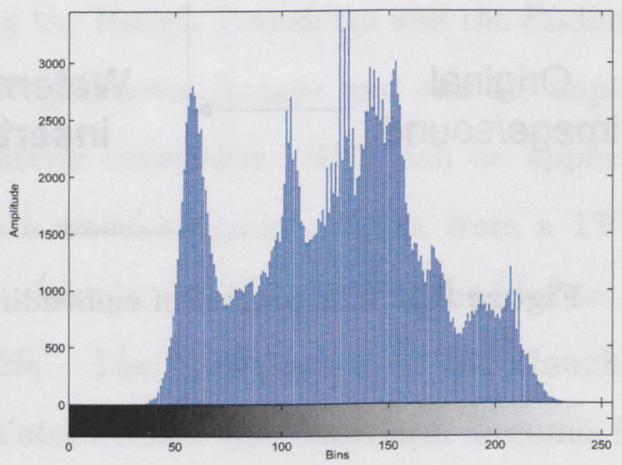

(f)

Figure 5.2: (a),(b): Image with no watermark embedded and its histogram, (c): time domain representation of the linear chirp (watermark), (d): TFD of the linear chirp, (e): the image in (a) with watermark embedded, and (f): its histogram. 
space has to satisfy some parametric constraint. The constraint could be a line or a curve in the image space depending on the directional pattern to be detected. If the feature to be detected is a line, it can be represented as:

$$
y_{i}=m x_{i}+b
$$

in which, $\left(y_{i}, x_{i}\right)$ is the point the line passing through in the image plane and $(m, b)$ represents the parameter space. For a fixed point $\left(y_{i}, x_{i}\right)$ in the image space, varying the value of $b$ results in different values for $m$. Plotting the corresponding values of parameters $m$ and $b$ in the $(m, b)$ plane results in a curve in the Hough space. Each line or point in the Hough space can be translated into a point or a line in the image space respectively. The problem with this representation of the line is the unbounded nature of parameter $b$ and $m$ which affects the practical implementation of the transform. The alternate way to represent the line is to use the form:

$$
\rho=x_{i} \cos \theta+y_{i} \sin \theta
$$

where $\rho$ is the distance from the origin to the line to be detected in the image space and $\theta$ is the angle between the $x$ axis and the direction perpendicular to the line to be detected (Figure 5.3).

With this new representation, the parameter $\theta$ is bounded within the interval $[0, \pi] . \mathrm{Pa}-$ rameter $\rho$ is bounded by the greater of rows and columns to $\pm N / \sqrt{2}$ in the parameter space, where $N$ is the size of the image. This alternative method makes it possible to implement the algorithm in practice. The Hough Transform shows its effectiveness in detecting linear patterns in many applications. However, it can be used for binary images only. To detect the linear chirp embedded as watermark message in audio or image, the Hough Transform has to be applied on the TFD of the chirp. But the TFD itself is a gray level image, not a binary image. Hence, the solution to this problem is to combine the Hough Transform with the Radon Transform [30, 26].

The Radon Transform computes the projection of different angels of a two-dimensional 


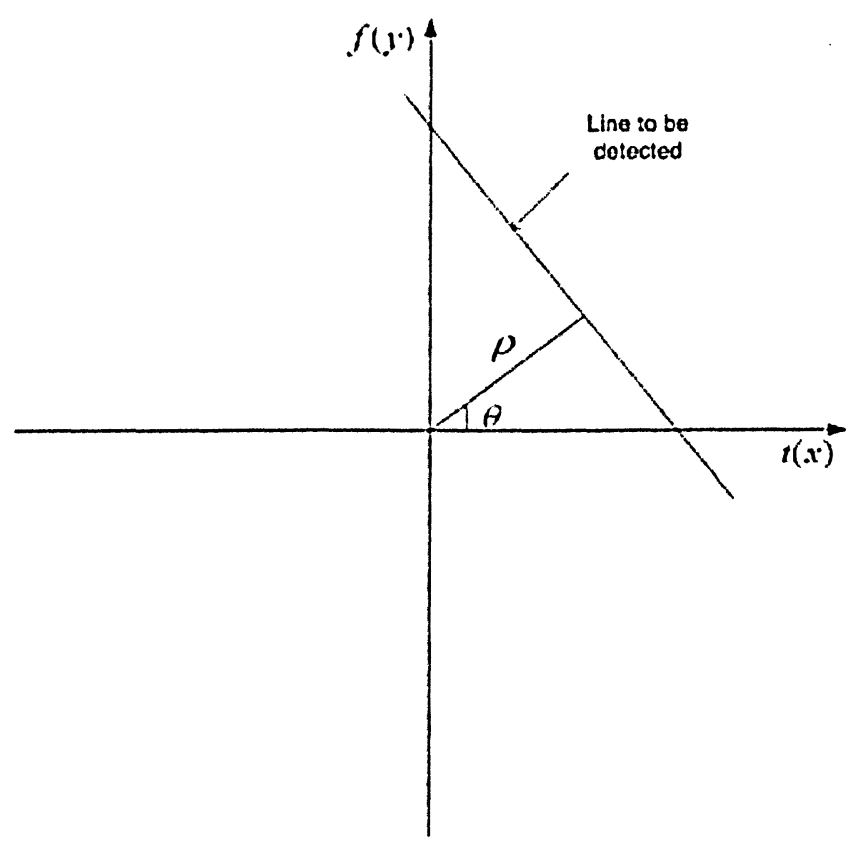

Figure 5.3: The image space

data distribution $\operatorname{TFD}(t, f)$ measured as line integrals along ray paths:

$$
\mathcal{R}(\rho, \theta)=\int_{-\infty}^{\infty} \int_{-\infty}^{\infty} T F D(t, f) \delta(\rho-(t \cos \theta+f \sin \theta)) d t d f .
$$

The Radon Transform is similar to the Hough Transform and a combination of the two transforms will allow the detection of the linear pattern of the chirp signal in the gray level TFD as well as quantization of the parameter space to reduce computational load.

To detect the linear chirp signal, the HRT can be applied on the WVD or the Spectrogram of the chirp $[25,26]$. To transform each point $\left(t_{i}, f_{i}\right)$ in the time-frequency plane to the corresponding curve in Hough space, the values of $\theta$ can be varied and the corresponding values of $\rho$ can be calculated. All the points belonging to the same line in the time-frequency plane will have their corresponding transform-curves in the Hough space intersect at the same point. This intersection will result in an energy peak in the Hough space. The coordinate and energy of the peak will help in making the decision if the chirp signal is detected.

To reduce computational load, the $\rho$ and $\theta$ axes can be quantized into a limited number of divisions, so only quantized values of those parameters will be involved in the calculation. 


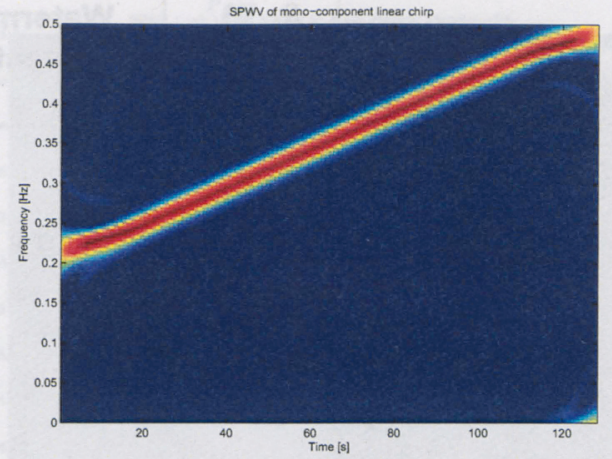

(a) SPWVD

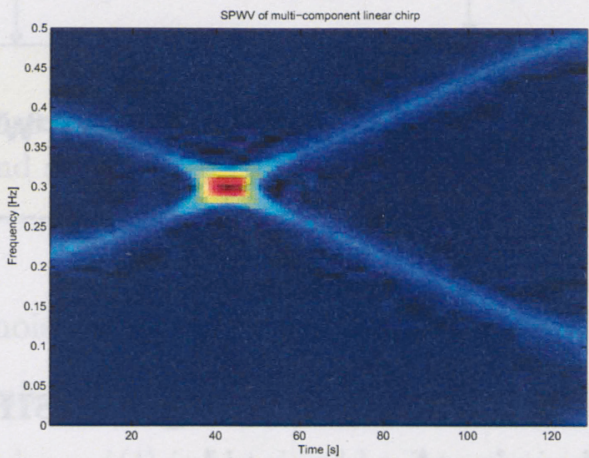

(c) SPWVD

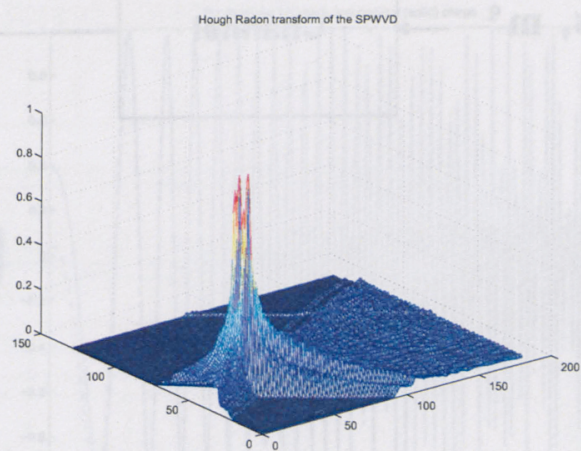

(b) HRT

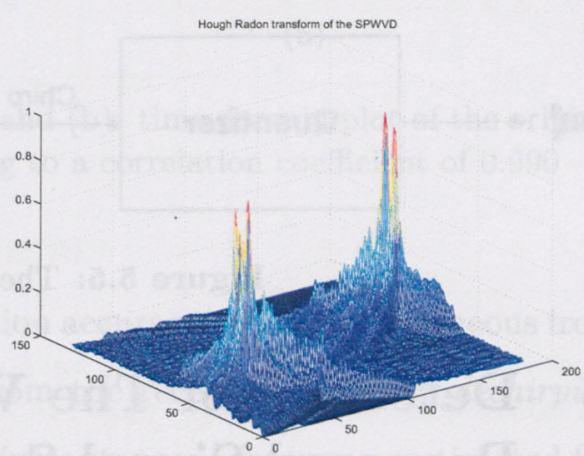

(d) HRT

Figure 5.4: SPWVD and the corresponding HRT of mono-component and multi-component linear chirp signals

Figure 5.4 shows the energy peaks in the Hough space resulted from taking the HRT of the SPWVD of the mono-component and multi-component linear chirps.

The HRT is a very effective method for detecting chirp signals. Even though more computationally effective than the maximum likelihood due to the quantization in the Hough space, HRT still suffers from the same computational draw back. Its complexity increases substantially with the size of the image and may not be applicable for real-time applications. The proposed method for detecting chirp signal is based on a combination of TFD and the DPPT. The method has lower computational complexity and its performance will be evaluated against HRT. 


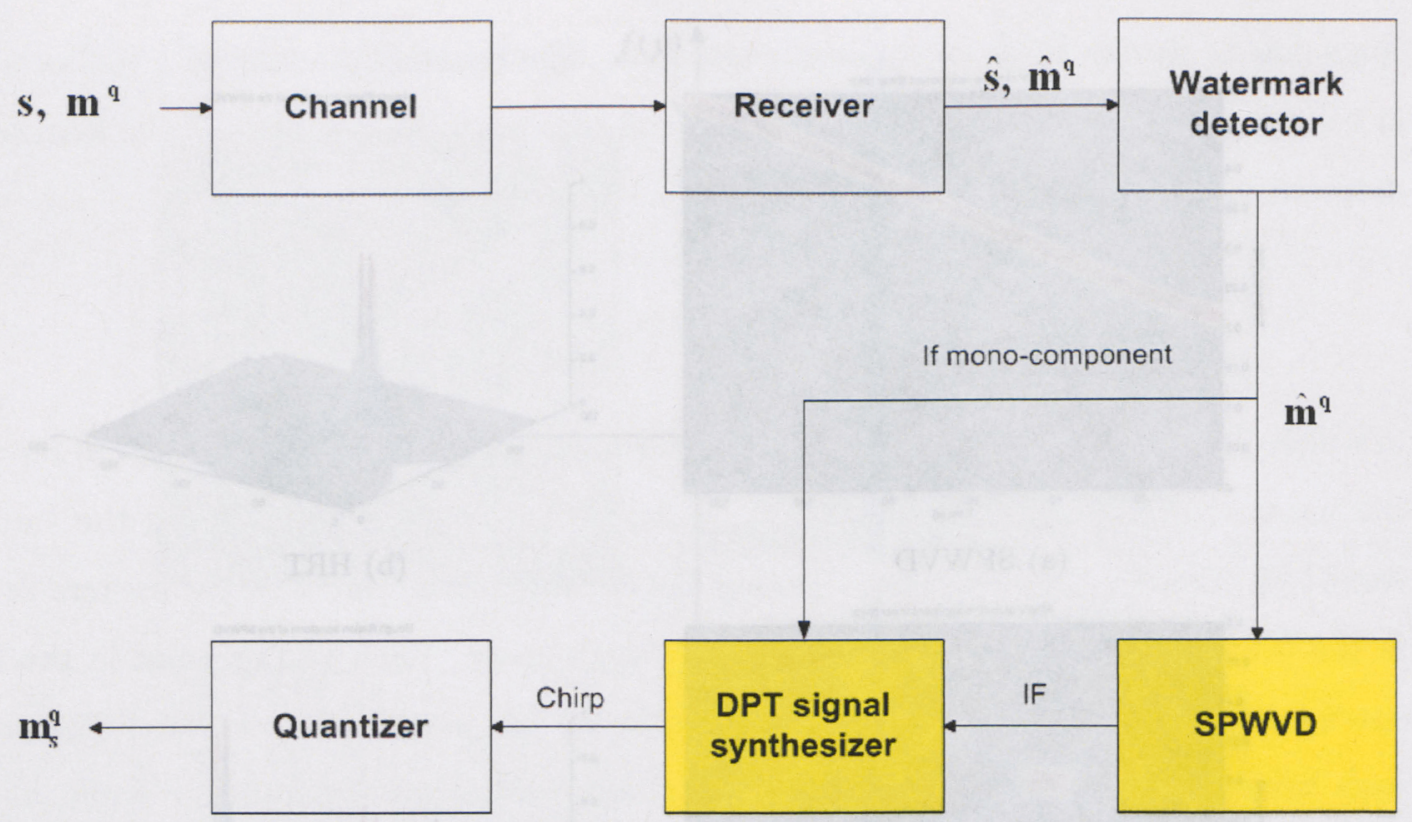

Figure 5.5: The watermark detection scheme

\subsection{Detection of The Watermark Message Using The Proposed Signal Synthesis Approach}

The method to embed and extract watermark message from audio and image signal is explained in $[25,26]$. This thesis work uses the proposed signal synthesis approach to synthesize the chirp message and compare it with the original chirp as a detection scheme. Let $\underline{\mathbf{m}}$ and $\mathbf{m}^{q}$ be the normalized chirp and its quantized version at the transmitter respectively. Let $\hat{\mathbf{m}}^{q}$ be the corrupted quantized chirp at the receiver. The detection process involves utilization of phase information which can be obtained from the TFD of the received signal. The SPWVD was used to calculate the TFD of $\hat{\mathbf{m}}^{q}$ instead of using the WVD or the Spectrogram as it had been done in other works. The detection scheme is illustrated as in Figure 5.5.

Since the discrete signal involved in the work is a quantized version of the chirp signal, its TFD consists of interference terms in addition to the linear component of the chirp. These interference terms' energy is smaller than the energy of the linear component, so they can be removed by applying a threshold on the TFD energy. The masking process helps to 


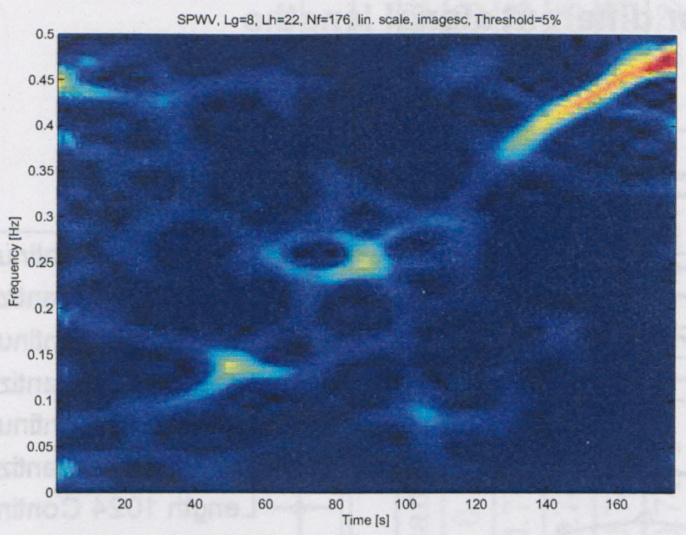

(a)

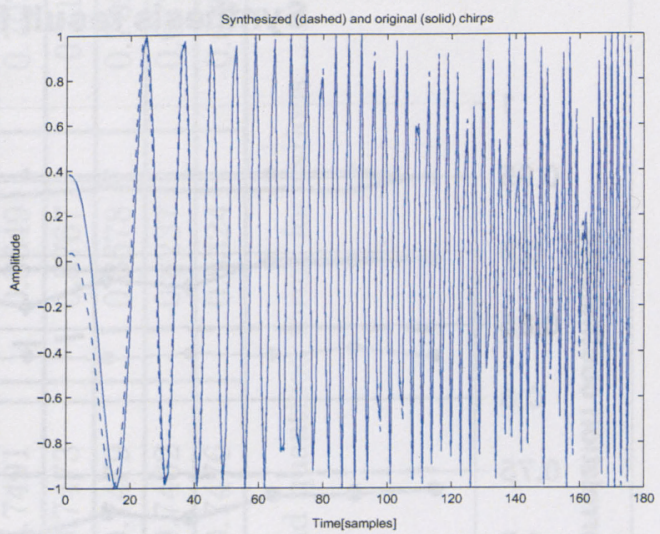

(b)

Figure 5.6: (a): TFD of the chirp with $20 \%$ BER, and (b): time domain plot of the original chirp (solid) and synthesized chirp (dashed) corresponding to a correlation coefficient of 0.990

remove noise in the TFD and increase the estimation accuracy of the instantaneous frequency. The mono-component of interest is extracted from the received signal by dechirping with $e^{-j \phi(t)}$, where $\phi(t)$ is obtained by integrating the instantaneous frequency estimated from the SPWVD. This extracted mono-component is then low-pass filtered and translated back into its original location by multiplying with $e^{j \phi(t)}$. The signal at this point can be considered a mono-component and is subjected to the DPPT algorithm as described in the previous sections.

The synthesized version of $\mathbf{m}^{q}$ is $\mathbf{m}_{s}^{q}$ obtained by quantization of $\underline{\hat{\mathbf{m}}}$, where $\underline{\hat{\mathbf{m}}}$ is the chirp estimated from the DPPT algorithm. Figure $5.8(\mathrm{~b})$ shows the original chirp $\underline{\mathbf{m}}$ and its estimated version $\underline{\hat{\mathbf{m}}}$ at BER of 5 percent. Figure $5.8(\mathrm{c})$ shows correlation coefficients between the pairs $(\underline{\mathbf{m}}, \underline{\hat{\mathbf{m}}}),\left(\mathbf{m}^{q}, \hat{\mathbf{m}}^{q}\right),\left(\mathbf{m}^{q}, \hat{\mathbf{m}}_{s}^{q}\right)$ and they are used as a standard to evaluate the effectiveness of the method. Figure 5.6 shows the detection result for a linear chirp with BER of $20 \%$, the correlation coefficient between the synthesized and referenced chirp is 0.990 .

The signal synthesis method proposed in Section 4.1 is proposed for detecting and separating individual signal components from a multi-component signal. In the multimedia 


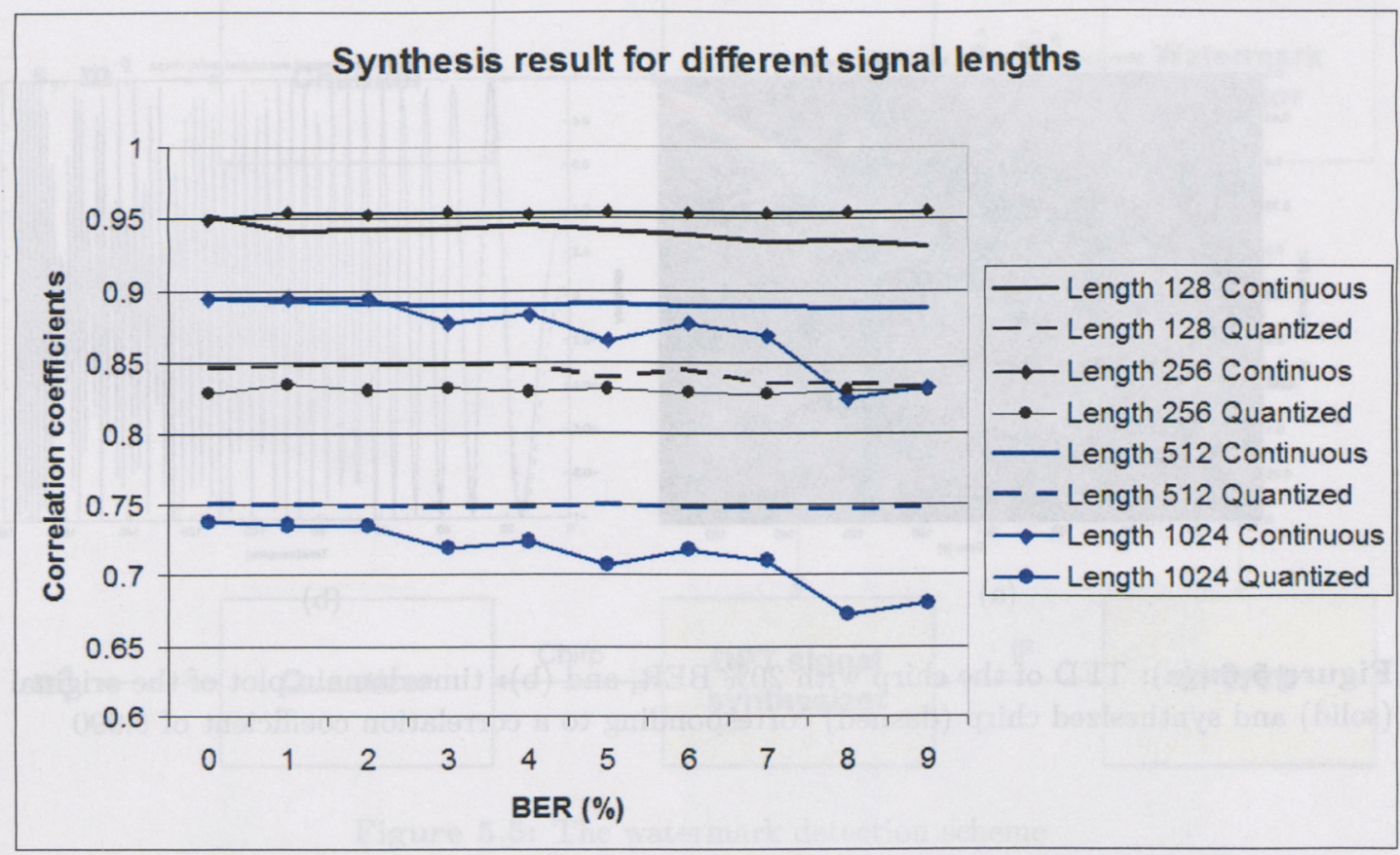

Figure 5.7: Correlation coefficients between the synthesized and referenced chirps in continuous and quantized forms for various length of the signals

watermark application within the scope of this thesis, each multimedia file is embedded with only one linear chirp message. Since the watermark message is a mono-component linear chirp, it can be detected by applying the DPPT algorithm directly after being extracted from the watermarked audio or image. The purpose of taking the SPWVD of the watermark message is to reduce the effect of noise on the signal and to extract the individual signal components out of the original multi-component message. Since the watermark is a monocomponent signal, operation involving the SPWVD as well as taking its first moment can be dropped. As a result, the detection algorithm will become faster because of the reduction in computational complexity.

As mentioned in the previous section, the accuracy of the detection algorithm depends on how precise the synthesized signal is compared to the referenced signal. The estimation of instantaneous frequency contributes significantly to the accuracy of the synthesized signal. 


\begin{tabular}{|c|c|c|c|c|c|c|c|c|}
\hline \multirow{2}{*}{ BER (\%) } & \multicolumn{9}{|c|}{ Signal length } \\
\cline { 2 - 8 } & \multicolumn{2}{|c|}{$\mathbf{1 2 8}$} & \multicolumn{2}{c|}{$\mathbf{2 5 6}$} & \multicolumn{2}{c|}{$\mathbf{5 1 2}$} & \multicolumn{2}{c|}{$\mathbf{1 0 2 4}$} \\
\cline { 2 - 9 } & Continuous & Quantized & Continuous & Quantized & Continuous & Quantized & Continuous & Quantized \\
\hline 0 & 0.9524 & 0.8455 & 0.9492 & 0.828 & 0.8948 & 0.75 & 0.8947 & 0.7384 \\
\hline 1 & 0.9423 & 0.8478 & 0.9547 & 0.8345 & 0.8926 & 0.7486 & 0.8945 & 0.7352 \\
\hline 2 & 0.9429 & 0.847 & 0.9532 & 0.8304 & 0.8908 & 0.7478 & 0.8945 & 0.7343 \\
\hline 3 & 0.9434 & 0.8473 & 0.9552 & 0.8319 & 0.8898 & 0.7468 & 0.8768 & 0.719 \\
\hline 4 & 0.9454 & 0.8472 & 0.9534 & 0.829 & 0.8896 & 0.7474 & 0.8827 & 0.7241 \\
\hline 5 & 0.9417 & 0.8393 & 0.9549 & 0.8312 & 0.8910 & 0.7491 & 0.8649 & 0.7079 \\
\hline 6 & 0.9385 & 0.8441 & 0.9537 & 0.8282 & 0.8892 & 0.7473 & 0.8767 & 0.718 \\
\hline 7 & 0.9338 & 0.8348 & 0.9540 & 0.8277 & 0.8888 & 0.7479 & 0.8678 & 0.7098 \\
\hline 8 & 0.9353 & 0.8344 & 0.9546 & 0.8305 & 0.8879 & 0.7462 & 0.8237 & 0.6723 \\
\hline 9 & 0.9315 & 0.8317 & 0.9554 & 0.8302 & 0.8883 & 0.7476 & 0.8324 & 0.6804 \\
\hline
\end{tabular}

Table 5.1: Correlation coefficient between the synthesized and referenced chirp in continuous and quantized forms for various length of the signals 


\begin{tabular}{|c|c|c|c|c|}
\hline \multirow{2}{*}{ BER (\%) } & \multicolumn{2}{|c|}{ With IF estimation } & \multicolumn{2}{c|}{ Without IF estimation } \\
\cline { 2 - 5 } & Continuous & Quantized & Continuous & Quantized \\
\hline 0 & 0.9534 & 0.8515 & 0.9889 & 0.8861 \\
\hline 1 & 0.9497 & 0.8416 & 0.9889 & 0.8898 \\
\hline 2 & 0.9463 & 0.8367 & 0.9884 & 0.8925 \\
\hline 3 & 0.9433 & 0.8333 & 0.9859 & 0.8901 \\
\hline 4 & 0.9441 & 0.8346 & 0.9854 & 0.8886 \\
\hline 5 & 0.9423 & 0.8332 & 0.9838 & 0.8879 \\
\hline 6 & 0.9427 & 0.8336 & 0.9835 & 0.8889 \\
\hline 7 & 0.9402 & 0.8298 & 0.9811 & 0.8836 \\
\hline 8 & 0.9414 & 0.8333 & 0.9814 & 0.8876 \\
\hline 9 & 0.9407 & 0.8298 & 0.9813 & 0.8856 \\
\hline
\end{tabular}

Table 5.2: Correlation coefficient between the synthesized and referenced chirp in continuous and quantized forms with and without the instantaneous frequency estimation process

Since the instantaneous frequency estimation step can be skipped, the contribution of the error it can possibly create is removed in the final synthesis output. The correlation between the synthesized and referenced chirp signal is therefore improved. The simulation is done for the detection of chirp signals with different bit error rates. The purpose of the simulation is to compare the detection limit of the HRT-based and chirp-synthesis-based detectors in terms of the maximal BER at which these detectors can detect the chirp. Therefore, it is easier and more convenient to add the error to the random positions of the chirp signals to obtain the desired BERs in a controllable way. The analysis of the effect of intentional and non-intentional attacks was already done thoroughly in [25]. Table 5.1 and Figure 5.7 show the correlation coefficients between the referenced chirp and the version of the chirp synthesized using the proposed technique in Section 4.1. As expected, the error of the synthesis procedure increases as the length of the chirp and the bit error rate (BER) increase. Table 5.2 and Figure 5.9 show the result of the chirp detection on the same signal with and without the instantaneous frequency estimation process through SPWVD. 


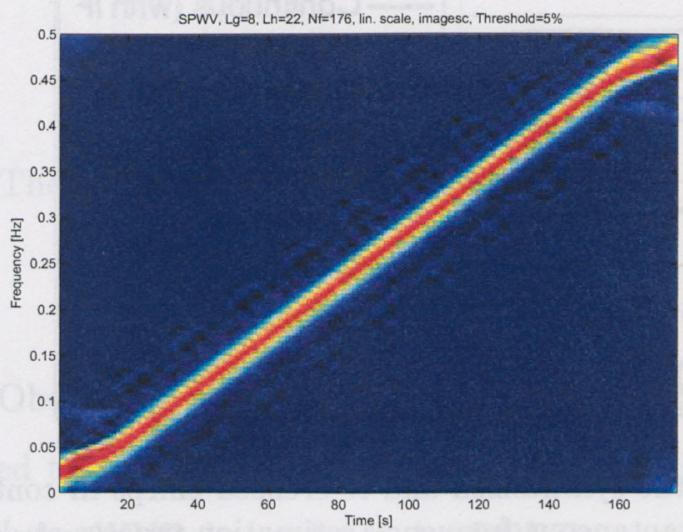

(a)

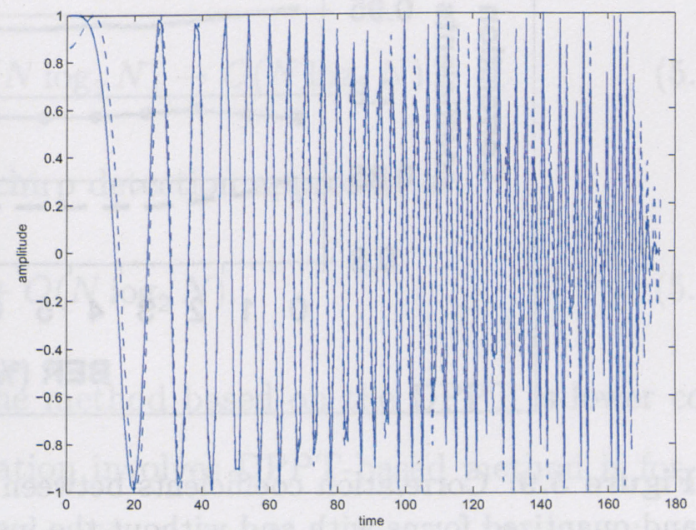

(b)

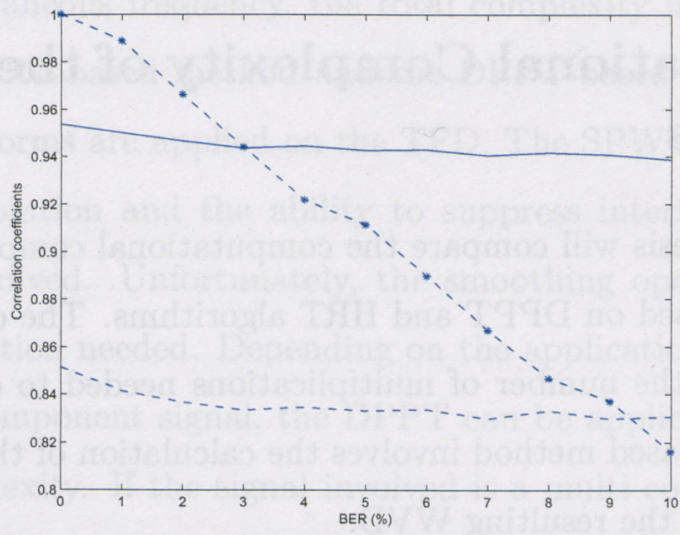

(c)

Figure 5.8: (a): TFD of the chirp, (b): time domain plot of the original chirp (solid) and synthesized chirp (dashed) corresponding to a correlation coefficient of 0.94 at $5 \%$ BER, and (c): correlation coefficients at different BER between the original and synthesized chirp (solid), between their quantized versions (dashed), and between the quantized original chirp and quantized chirp at the receiver (dash-star) 


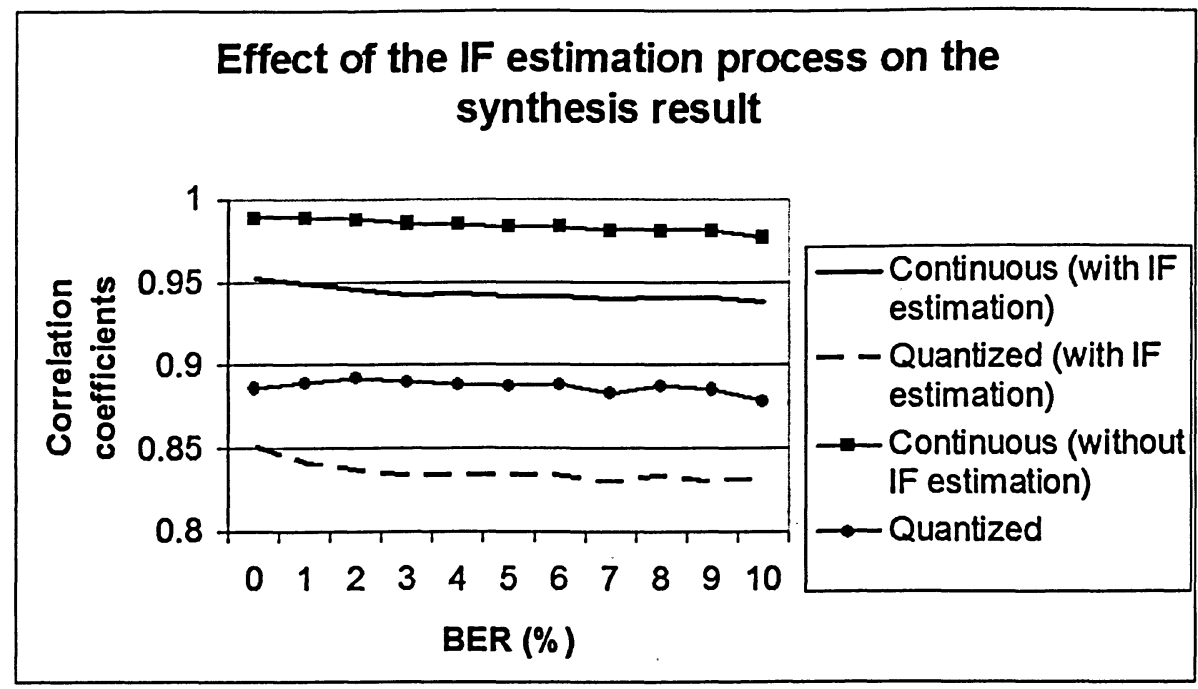

Figure 5.9: Correlation coefficients between the synthesized and referenced chirps in continuous and quantized forms with and without the instantaneous frequency estimation process at different BERs

\subsection{Computational Complexity of the Chirp Detection Methods}

This section of the thesis will compare the computational complexity (CC) of the the chirp detection methods based on DPPT and HRT algorithms. The computational complexity is determined based on the number of multiplications needed to detect a linear chirp having length $N$. The HRT-based method involves the calculation of the WVD [53] and taking the standard HRT [54] on the resulting WVD:

$$
\begin{aligned}
& C C(W V D)=O\left(N^{2} \log _{2} N\right) \\
& C C(H R T)=O\left(N^{2} t\right)
\end{aligned}
$$

where $t$ is the number of bins used for the quantization of each axis in the Hough space $(t<\sqrt{2} N)$. The total complexity is:

$$
O\left(N^{2} \log _{2} N\right)+O\left(N^{2} t\right)
$$

The DPPT-based method estimates the signal parameter from the TFD (WVD for example). It involves the WVD calculation of the chirp signal and taking the DPPT of the 
estimated chirp. The DPPT is calculated by first taking the ambiguity function and then taking the Fourier Transform of the ambiguity function. The computational complexity of the ambiguity function calculation and the fast Fourier Transform are $O(N)$ and $O\left(N \log _{2} N\right)$ respectively. Therefore,

$$
C C(D P P T)=O(N)+O\left(N \log _{2} N\right) \rightarrow O\left(N \log _{2} N\right) .
$$

The total complexity of the DPPT-based chirp detection approach is:

$$
O\left(N^{2} \log _{2} N\right)+O\left(N \log _{2} N\right) .
$$

Obviously, computational complexity of the method based on the DPPT is lower compared to the HRT-based method. The calculation involves DPPT-based method is for the case the WVD is used. If the DPPT is applied directly on the signal without using the TFD to estimate instantaneous frequency, the total complexity is only $O\left(N \log _{2} N\right)$. The similarity between the HRT-based method and the DPPT-based method proposed in this thesis is that both transforms are applied on the TFD. The SPWVD is known to have high joint time-frequency resolution and the ability to suppress interference terms due to the smoothing operation involved. Unfortunately, the smoothing operation also increases the total amount of computation needed. Depending on the application at hand, if the chirp to be detected is a mono-component signal, the DPPT can be applied directly to it to reduce the computational complexity. If the signal involved is a multi-component signal, then the individual components have to be extracted first before the DPPT can be used. Because of this reason, the SPWVD should be used in place of the WVD. 


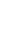




\section{Chapter 6}

\section{Conclusions}

\subsection{Conclusions}

\section{Time-Frequency signal synthesis}

The success of the proposed watermark detection technique depends considerably on the quality of the synthesized chirp. The accuracy of the synthesis process in turn depends on how precise the initial estimation of the instantaneous frequency is. The simulations performed on different types of signals and noise levels prove that among the TFDs whose kernel is signal-independent, the SPWVD is a good candidate for determining instantaneous frequency due to its advantageous kernel design. The kernel consists of two windows $g(t)$ and $h(t)$ for the independent smoothing operation in time and in frequency. The summary of the result is:

- The SPWVD has more versatility to adapt to different types of signal. Careful selection of the types and length of the windows can suppress interference terms with the least joint time-frequency resolution smearing.

- The SPWVD has high ability in interference term suppression because filtering of the interference term can be done in the time and frequency directions.

- Throughout the simulation, not only has the SPWVD provided good ability to remove interference terms, its performance is also less affected by noise. The simulations show that instantaneous frequency extracted from the SPWVD is highly correlated 
with the referenced instantaneous frequency even under very strong noise. Although the WVD has excellent resolution on mono-component signal, the correlation between instantaneous frequency estimated from the WVD and the referenced instantaneous frequency is decreased significantly under strong noise. Due to this reason, the SPWVD was chosen as the processing TFD in the proposed chirp detection for the monocomponent and multi-component chirps. In the watermark application, the received chirp is sometimes heavily corrupted because of intentional and non-intentional attacks. Therefore, using the SPWVD in this case is a better choice.

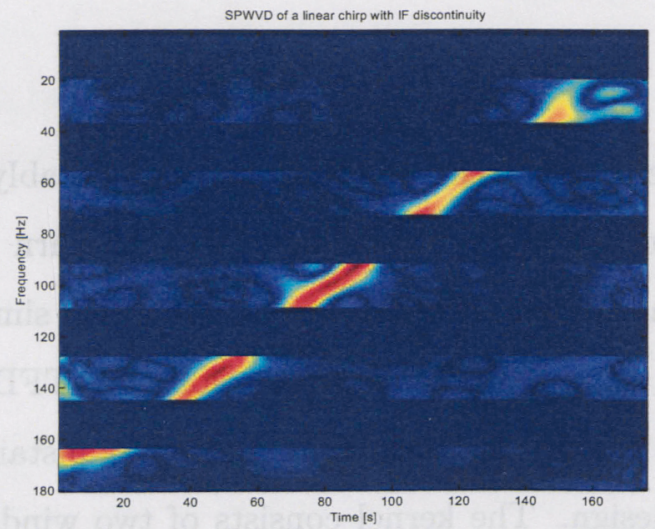

(a)

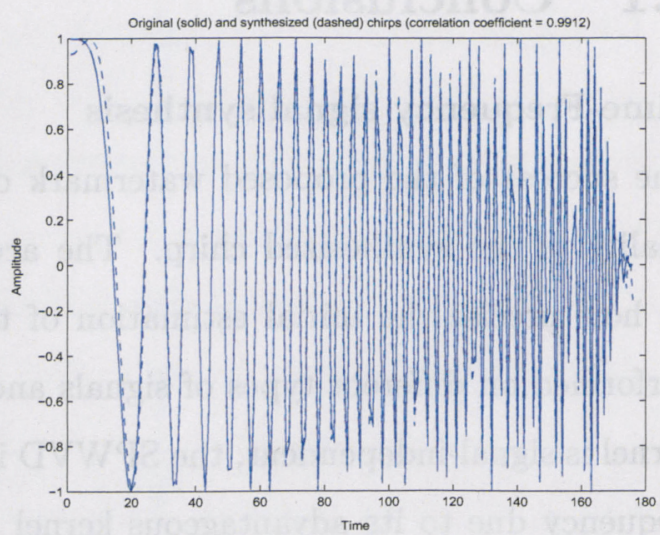

(b)

Figure 6.1: (a): TFD of the chirp with discontinuity in its instantaneous frequency law, and (b): time domain plot of the original chirp (solid) and synthesized chirp (dashed) corresponding to a correlation coefficient of 0.9912

In this study, a new signal synthesis technique was proposed. The technique combines the use of the DPPT and the TFD and can be used in many applications such as time-frequency filtering, time-frequency noise suppression and signal design. In addition, time-frequency signal synthesis can be used in radar and telecommunication applications to detect the presence of the chirp signal. One important application of the proposed signal synthesis technique is the instantaneous frequency estimation of a signal. The instantaneous frequency itself is an indispensable tool in the analysis of time-varying signal. For example, in order to determine 
the instantaneous frequency of the mono-components of a multi-component signal, apply the proposed technique on the multi-component signal to synthesize the individual components. When the precise mono-components are available, their instantaneous frequency can be easily calculated from their SPWVD.

The proposed signal synthesis technique can be applied to synthesize the chirp signal from just a portion of its instantaneous frequency in the TFD. The DPPT algorithm is applied on the TFD to calculate the phase coefficients of the signal. Then the time domain signal can be recovered from the coefficients and the time period corresponding to the presence of the signal in the TFD using the chirp formula. In addition, the proposed technique not only can detect the chirp message but also has the ability of error correction and reconstruction of the original chirp. It can detect and synthesize the chirp signal from a distorted TFD having discontinuity in its instantaneous frequency trajectory. Figure 6.1 shows the TFD of a chirp signal and the corresponding synthesized chirp. The instantaneous frequency of the signal in the TFD was intentionally destroyed so that it contains discontinuity. Both the referenced and synthesized chirps are highly correlated despite the corruption in the instantaneous frequency. Therefore the technique possibly can be used for error correction application. When the code or the chirp is corrupted under various conditions of the environment, it can be recovered safely using the synthesis method.

In this thesis, the proposed signal synthesis method was used in multimedia security application such as watermarking. The method is applied to detect the chirp embedded in multimedia files as a watermark message. The proposed detection method is used as a replacement for the HRT-based detection method in previous works. The signal synthesis based on DPPT has much lower computational complexity and the improvement in computation is considered a contribution of the thesis.

\section{Watermark Detection}

The proposed technique, like the HRT-based method, can be used to detect the chirp message embedded in image and audio signals. The technique has the ability to detect the 
chirp signals whose corruption level is simulated at different BERs. The simulations show its robustness for detecting corrupted chirp signal with BER of up to $15 \%$. Since the watermark message is a linear frequency modulated signal, it is easily modeled using the DPPT. Therefore, the parameters of the chirp such as slope, initial phase, and initial frequency can be recovered easily and precisely.

The novelty of the new method is in the fact that it is very efficient in terms of computational complexity. The computational complexity and the accuracy of previously used HRT-based chirp detection method depend on the number of bins $t$ used in the Hough space. The number of bins $t$ must be large enough (small bin size) to ensure that the lines in the Hough space corresponding to different points in the TFD do not overlap. However, increasing the number of bins will reduce the speed of the algorithm. This is the trade off between speed and accuracy in the HRT-based approach. The total computational complexity of the previous HRT-based approach is $O\left(N^{2} \log _{2} N\right)+O\left(N^{2} t\right)$. This total complexity can be improved to only $\mathrm{O}\left(\mathrm{N} \log _{2} N\right)$ in the proposed DPPT-based technique in this thesis.

In this approach, most of the running time is spent on pre-processing steps such as thresholding the TFD, estimating instantaneous frequency to isolate the chirp signal $\hat{\mathbf{m}}^{q}$. If the DPPT is applied directly on $\hat{\mathbf{m}}^{q}$, the calculation time will be reduced significantly. The simulation shows that, for a specific chirp length of 176 , the proposed chirp detection method is three times faster than the HRT-based approach using $128 X 128$ bins in the Hough space. So with the new proposed method, trade-off problem between speed and accuracy in HRT-based method is solved. Faster detection is thus allowed together with accuracy. This is also the motivation for the proposed approach for chirp detection in a real-time application such as image watermarking of multimedia data.

The novel technique can be used to detect watermark message on images with different BERs. The simulation showed high correlation $(>0.9)$ between $\underline{\mathbf{m}}$ and $\underline{\hat{\mathbf{m}}}$. As a result of the quantization process, the correlation between $\mathbf{m}^{q}$ and $\hat{\mathbf{m}}_{s}^{q}$ is lower than the previous case but it is still larger than 0.8 at different BER values within the BER limit. These correlation coefficients can help to confidently make decision on the transmitted chirp's properties. In 
the simulation, the watermark message embedded in the images is a mono-component linear FM chirp. However, the method can be extended to detect image watermark messages consisting of multi-component linear chirps.

The limit of the BER for a safe detection in the proposed algorithm is $15 \%$. This value is a bit lower compared to the $20 \%$ limit in the HRT-based technique. In the HRTbased approach, the detection result is better with increasing chirp length. In the proposed technique, a contradiction resulted regarding the chirp length. As the chirp length increases, the accuracy decreases because any possible error in the estimated phase coefficients will propagate through the length of the signal. When BER is greater than $15 \%$, the proposed method can't always detect the chirp message. Besides the difference in the BER limit for detection and in the relation between the chirp length and accuracy, the proposed method also has the error correction ability as the HRT-based method.

\subsection{Related Future Work}

The goal in future work is to increase the accuracy of the synthesis algorithm. The limitation of the current method is low-BER detection limit. This means that the performance of the synthesis technique reduces when the effect of noise becomes stronger. The following could be done to improve the accuracy of the synthesis method:

- In the preprocessing of the TFD before the chirp signal is synthesized, the TFD (SPWVD) of the signal is thresholded to remove noise. The current threshold setting is at 0.8 of the maximal energy of the TFD. This value is obtained empirically. A more detailed and systematic analysis of the effect of noise on the signal should be done so the noise threshold of the TFD can be determined adaptively based on the condition of the signal.

- In addition, the future synthesis method should be extended to include the ability to efficiently isolate and synthesize the individual components from a multi-component signal. The goal is to be able to separate the mono-component instantaneous frequen- 
cies from the SPWVD. The separated instantaneous frequency does not have to be precise because DPPT can be applied on the separated instantaneous frequency to get a final synthesized version of the mono-component. The approach in [37] or any others can be used to estimate the instantaneous frequency of the mono-components. This new ability can expand the application of the synthesis method even further. For example, multiple linear chirps can be embedded in the same multimedia file to increase the information content in the watermark message. 


\section{Appendix A}

\section{List of Publications}

In this section, the publications resulted from this research work are presented.

\section{Journals}

- L. Le and S. Krishnan, "Time-Frequency Signal Synthesis and its Application in Multimedia Watermark Detection," submitted to, EURASIP Journal on Applied Signal Processing, under second-round review. 


\section{Appendix B}

\section{List of Acronyms}

$\begin{array}{ll}\text { BER } & \text { Bit Error Rate } \\ \text { CC } & \text { Computational Complexity } \\ \text { CT } & \text { Chirplet Transform } \\ \text { CWD } & \text { Choi-Williams Distribution } \\ \text { DCT } & \text { Discrete Cosine Transform } \\ \text { DPPT } & \text { Discrete Polynomial Phase Transform } \\ \text { EDTWD } & \text { Extended Discrete Time Wigner Distribution } \\ \text { EEG } & \text { Electroencephalogram } \\ \text { EMG } & \text { Electromyogram } \\ \text { FIR } & \text { Finite Impulse Response } \\ \text { FM } & \text { Frequency Modulation } \\ \text { FSK } & \text { Frequency Shift Keying } \\ \text { HRT } & \text { Hough Radon Transform } \\ \text { IF } & \text { Instantaneous Frequency } \\ \text { IIPA } & \text { International Intellectual Property Alliance } \\ \text { IIR } & \text { Infinite Impulse Response } \\ \text { JND } & \text { Just Noticeable Difference } \\ \text { MCE-TFD } & \text { Minimum Cross Entropy Time-Frequency Distribution } \\ \text { MPTFD } & \text { Matching Pursuit Time-Frequency Distribution } \\ \text { PN } & \text { Pseudo-Random } \\ \text { PWVD } & \text { Pseudo Wigner-Ville Distribution } \\ \text { SNR } & \text { Signal to Noise Ration } \\ \text { SP } & \text { Spectrogram } \\ \text { SPWVD } & \text { Smoothed Pseudo Wigner-Ville Distribution } \\ \text { STFT } & \text { Short-Time Fourier Transform } \\ \text { TFD } & \text { Time-Frequency Distribution } \\ \text { WVD } & \text { Wigner-Ville Distribution } \\ \end{array}$




\section{Bibliography}

[1] B. Boashash. Time-frequency signal analysis. Longman Cheshire, Wiley Halsted Press, Melbourne, Australia, 1992.

[2] F. Hlawatsch and G.F. Boudreaux-Bartels. Linear and quadratic time-frequency signal representations. IEEE Transactions on Acoustics, Speech, and Signal Processing, 34(3):442 - 451, Jun 1986.

[3] G.F. Boudreaux-Bartels and T. Parks. Time-varying filtering and signal estimation using wigner distribution synthesis techniques. IEEE Signal Processing Magazine, 9(2):2167, April 1992.

[4] L. Cohen. Time-frequency distributions- a review. Proceedings of the IEEE, 77:941-981, 1989.

[5] J. Jeong and W.J. Williams. On the cross-terms in spectrograms. Proc. IEEE International Symposium on Circuits and Systems, pages (3):1565 - 1568, May 1990.

[6] T.A.C.M. Claasen and W.F.G. Mecklenbrauker. The wigner distribution - a tool for time-frequency signal analysis, part 2: Discrete-time signals. Phillips Journal of Research, 35(4/5):276-300, 1980.

[7] D. Griffin and L. Jae. Signal estimation from modified short-time fourier transform. IEEE Transactions on Acoustics, Speech, and Signal Processing, 32(2):236 - 243, Apr 1984. 
[8] S. Sussman. Least-square synthesis of radar ambiguity functions. IEEE Transactions on Information Theory, 8(3):246-254, Apr 1962.

[9] K.B. Yu and S. Cheng. Signal synthesis from pseudo-wigner distribution and applications. IEEE Signal Processing Magazine, 35(9):1289 - 1302, Sep 1987.

[10] W. Krattenhaler and F. Hlawatsch. Two signal synthesis algorithms for pseudo wigner distribution. Proc. IEEE International Conference on Acoustics, Speech, and Signal Processing (ICASSP), pages (3):1550 - 1553, Apr 1998.

[11] W. Krattenhaler and F. Hlawatsch. Improved signal synthesis from pseudo-wigner distribution. IEEE Transactions on Signal Processing, 39(2):506 - 509, Feb 1991.

[12] W. Krattenhaler and F. Hlawatsch. Phase matching algorithms for wigner-distribution signal synthesis. IEEE Transactions on Signal Processing, 39(3):612 - 619, Mar 1991.

[13] W. Krattenhaler and F. Hlawatsch. Bilinear signal synthesis. IEEE Transactions on Signal Processing, 40(2):352 - 363, Feb 1992.

[14] W. Krattenhaler and F. Hlawatsch. Time-frequency design and processing of signals via smoothed wigner distributions. IEEE Transactions on Signal Processing, 41(1):278, Jan 1993.

[15] W. Krattenhaler and F. Hlawatsch. General signal synthesis algorithms for smoothed versions of wigner distribution. Proc. IEEE International Conference on Acoustics, Speech, and Signal Processing (ICASSP), pages (3):1611 - 1614, Apr 1990.

[16] J. Jeong and W. Williams. Time-varying filtering and signal synthesis. Time-Frequency Signal Analysis - Methods and Applications, 1995.

[17] Y. Zhang, M.G. Amin, and A.R. Lindsey. Anti-jamming gps receivers based on bilinear signal distributions. Proc. Military Communications Conference MILCOM, pages (2):1070 - 1074, Oct 2001. 
[18] W. Mu, Y. Zhang, and M.G. Amin. Bilinear signal synthesis in array processing. Proc. IEEE International Conference on Acoustics, Speech, and Signal Processing (ICASSP), pages (6):3577 - 3580, May 2001.

[19] W. Mu, Y. Zhang, and M.G. Amin. Bilinear signal synthesis in array processing. IEEE Transactions on Signal Processing, 51(1):90 - 100, May 2003.

[20] W. Mu, Y. Zhang, and M.G. Amin. Spatial and time-frequency signature estimation of nonstationary sources. Proceedings of the 11th IEEE Signal Processing Workshop on Statistical Signal Processing, pages 313 - 316, Aug 2001.

[21] A. Kandangath. Jamming mitigation techniques for spread spectrum communication systems. EEE 598A: Signal Processing For Wireless Communications, Dec 2003.

[22] N. Stevenson, L. Rankine, M. Mesbah, and B. Boashash. Newborn eeg seizure simulation using time-frequency signal synthesis. WDIC 2005 APRS Workshop on Digital Image Computing. Theme: Pattern Recognition and Imaging for Medical Applications, page CDROM, 2005.

[23] www.iipa.com.

[24] www.digimarc.com.

[25] A. Ramalingam and S. Krishnan. A novel robust image watermarking using a chirp based technique. Proc. Canadian Conference on Electrical and Computer Engineering, pages 1889-1892, May 2004.

[26] S. Erkucuk, S. Krishnan, and M. Zeytinoglu. Robust audio watermarking using a chirp based technique. Proc. International Conference on Multimedia and Expo, 2003. ICME '03, pages II - 513-16, July 2003.

[27] L. Cohen. The uncertainty principle in signal analysis. Proceedings of the IEEE-SP International Symposium on Time-Frequency and Time-Scale Analysis, pages 182 185, Oct 1994. 
[28] F. Hlawatsch, T.G. Manickam, R.L. Urbanke, and W. Jones. Smoothed pseudo wigner distribution, choi-williams distribution, and cone kernel representation: Ambiguity domain analysis and experimental comparison. Signal Processing, 43(2):149-168, May 1995.

[29] TFTB toolbox. http://iut-saint-nazaire.univ-nantes.fr/ auger/tftb.html.

[30] S. Krishnan. Instantaneous mean frequency estimation using adaptive time-frequency distributions. Proc. Canadian Conference on Electrical and Computer Engineering, pages 141-146, May 2001.

[31] S.G. Mallat and Z. Zhang. Matching pursuit with time-frequency dictionaries. IEEE Transactions on Signal Processing, 41(12):3397-3415, 1993.

[32] A. Francos and M. Porat. Analysis and synthesis of multicomponent signals using positive time-frequency distributions. IEEE Transactions on Signal Processing, 47(2):493504, Feb. 1999.

[33] S. Peleg and B. Friedlander. Multicomponent signal analysis using the polynomial-phase transform. IEEE Transactions on Aerospace and Electronic Systems, 32(1):378-387, Jan 1996.

[34] S. Peleg and B. Friedlander. The discrete polynomial-phase transform. IEEE Transactions on Signal Processing, 43(8):1901-1914, August 1995.

[35] S. Peleg and B. Friedlander. A technique for estimating the parameters of multiple polynomial phase signals. In Time-Frequency and Time-Scale Analysis. Proceedings of the IEEE-SP International Symposium, pages 119 - 122, Oct 1992.

[36] B. Boashash. Estimating and interpreting the instantaneous frequency of a signal. ii. algorithms and applications. Proceedings of the IEEE, 80(4):540-568, April 1992. 
[37] B. Barkat and K. Abed-Meraim. A blind components separation procedure for fm signal analysis. Proc. IEEE International Conference on Acoustics, Speech, and Signal Processing, pages (2):1425 - 1428, 2002.

[38] E. Chassande-Mottin and P. Flandrin. On the time-frequency detection of chirps. $A p-$ plied and Computational Hamornic Analysis, 6:252-281, 1999.

[39] S. Kay and G. Boudreaux-Bartels. On the optimality of the wigner distribution for detection. Proc. IEEE International Conference on Acoustics, Speech, and Signal Processing (ICASSP), pages 1017-1020, Apr 1985.

[40] M. Morvidone and B. Torresani. Time scale approach for chirp detection. International Journal of Wavelets, Multiresolution and Information Processing, 1(1):19-49, 2003.

[41] G. Wang and X.G. Xia. An adaptive filtering approach to chirp estimation and isar imaging of maneuvering targets. In The Record of the IEEE 2000 International Radar Conference, 2000, pages 481 - 486, May 2000.

[42] A.B. Doser and G.D.T. Schuller. Time/frequency techniques for signal feature detection. Proc. Conference Record of the Thirty-Third Asilomar Conference on Signals, Systems, and Computers, pages (1):452 - 456, Oct 1999.

[43] J.S. Dhanoa, E.J. Hughes, and R.F Ormondroyd. Simultaneous detection and parameter estimation of multiple linear chirps. Proc. IEEE International Conference on Acoustics, Speech, and Signal Processing (ICASSP), pages VI - 129-32 vol.6, Apr 2003.

[44] W. Li. Wigner distribution method equivalent to dechirp method for detecting a chirp signal. IEEE Transactions on Acoustics, Speech, and Signal Processing, 35(8):1210 1211, Aug 1987.

[45] L. Sin-Joo and J. Sung-Hwan. A survey of watermarking techniques applied to multimedia. Proc. IEEE International Symposium on Industrial Electronics, pages I:272 277, Jun 2001. 
[46] B.G. Mobasseri. Digital watermarking in joint time-frequency domain. Proc. International Conference on Image Processing, pages III-481 - III-484, Jun 2002.

[47] F. Sattar and B. Barkat. A new time-frequency based private fragile watermarking scheme for image authentication. Proc. Seventh International Symposium on Signal Processing and Its Applications, pages II:363 - 366, Jul 2003.

[48] C.I. Podilchuk and W. Zeng. Image-adaptive watermarking using visual models. IEEE Journal on Selected Areas in Communications, 16:525-539, May 1998.

[49] M. Morvidone and B. Torrsani. Variations on hough-wavelet transforms for timefrequency chirp detection. In Proceedings of the Wavelet $X$ conference, SPIE symposium, pages 181-195, San Diego, 2003.

[50] B.D. Carlson, E.D. Evans, and S.L. Wilson. Search radar detection and track with the hough transform. i. system concept. IEEE Transactions on Aerospace and Electronic Systems, 30(1):102 - 108, Jan 1994.

[51] B.D. Carlson, E.D. Evans, and S.L. Wilson. Search radar detection and track with the hough transform. ii. detection statistics. IEEE Transactions on Aerospace and Electronic Systems, 30(1):109 - 115, 1994.

[52] B.D. Carlson, E.D. Evans, and S.L. Wilson. Search radar detection and track with the hough transform. iii. detection performance with binary integration. IEEE Transactions on Aerospace and Electronic Systems, 30(1):116 - 125, Jan 1994.

[53] F. Peyrin and R. Prost. A unified definition for the discrete-time, discrete-frequency, and discrete-time/frequency wigner distributions. IEEE Transactions on Acoustics, Speech, and Signal Processing, 34(4):858 - 867, Aug 1986.

[54] S. Pavel and S.G. Akl. Efficient algorithms for the hough transform on arrays with reconfigurable optical buses. In Proceedings of the 10th International Parallel Processing Symposium, pages 697 - 701, April 1996. 